\title{
Pulsating white dwarfs: new insights
}

\author{
Alejandro H. Córsico ${ }^{1,2}$. Leandro G. Althaus ${ }^{1,2}$. \\ Marcelo M. Miller Bertolami ${ }^{1,2}$. S. O. Kepler ${ }^{3}$
}

Received: 11 March 2019 / Published online: 3 September 2019

(c) Springer-Verlag GmbH Germany, part of Springer Nature 2019

\begin{abstract}
Stars are extremely important astronomical objects that constitute the pillars on which the Universe is built, and as such, their study has gained increasing interest over the years. White dwarf stars are not the exception. Indeed, these stars constitute the final evolutionary stage for more than $95 \%$ of all stars. The Galactic population of white dwarfs conveys a wealth of information about several fundamental issues and are of vital importance to study the structure, evolution and chemical enrichment of our Galaxy and its components-including the star formation history of the Milky Way. Several important studies have emphasized the advantage of using white dwarfs as reliable clocks to date a variety of stellar populations in the solar neighborhood and in the nearest stellar clusters, including the thin and thick disks, the Galactic spheroid and the system of globular and open clusters. In addition, white dwarfs are tracers of the evolution of planetary systems along several phases of stellar evolution. Not less relevant than these applications, the study of matter at high densities has benefited from our detailed knowledge about evolutionary and observational properties of white dwarfs. In this sense, white dwarfs are used as laboratories for astro-particle physics, being their interest focused on physics beyond the standard model, that is, neutrino physics, axion physics and also radiation from "extra dimensions", and even
\end{abstract}

\footnotetext{
$凶$ Alejandro H. Córsico

acorsico@fcaglp.unlp.edu.ar

Leandro G. Althaus

althaus@fcaglp.unlp.edu.ar

Marcelo M. Miller Bertolami mmiller@fcaglp.unlp.edu.ar

S. O. Kepler

kepler@if.ufrgs.br

1 Facultad de Ciencias Astronómicas y Geofísicas, Universidad Nacional de La Plata, Paseo del Bosque s/n, (1900), La Plata, Argentina

2 Instituto de Astrofísica La Plata, IALP, CONICET-UNLP, La Plata, Argentina

3 Departamento de Astronomia, Universidade Federal do Rio Grande do Sul, Av. Bento Goncalves 9500, Porto Alegre, RS 91501-970, Brazil
} 
crystallization. The last decade has witnessed a great progress in the study of white dwarfs. In particular, a wealth of information of these stars from different surveys has allowed us to make meaningful comparison of evolutionary models with observations. While some information like surface chemical composition, temperature and gravity of isolated white dwarfs can be inferred from spectroscopy, and the total mass and radius can be derived as well when they are in binaries, the internal structure of these compact stars can be unveiled only by means of asteroseismology, an approach based on the comparison between the observed pulsation periods of variable stars and the periods predicted by appropriate theoretical models. The asteroseismological techniques allow us to infer details of the internal chemical stratification, the total mass, and even the stellar rotation profile. In this review, we first revise the evolutionary channels currently accepted that lead to the formation of white-dwarf stars, and then, we give a detailed account of the different sub-types of pulsating white dwarfs known so far, emphasizing the recent observational and theoretical advancements in the study of these fascinating variable stars.

Keywords Stellar evolution $\cdot$ White dwarf stars $\cdot$ Stellar interiors $\cdot$ Stellar oscillations · Asteroseismology

\section{Contents}

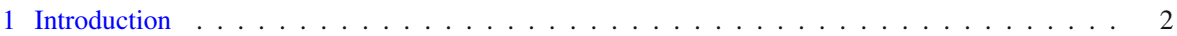

2 Evolutionary channels and uncertainties in progenitor evolution . . . . . . . . . . . 8

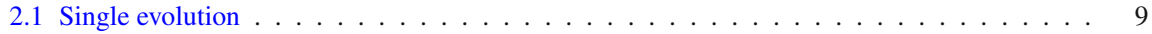

2.2 Binary evolution . . . . . . . . . . . . . . . . . . . . . . . 13

3 Asteroseismology of pulsating WDs and pre-WDs . . . . . . . . . . . . . . . . . . . . . . . . . . . . . . . .

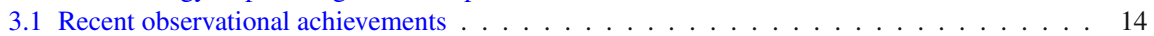

3.2 Asteroseismic approaches . . . . . . . . . . . . . . . . . . . . . . . . 29

3.3 Massive and ultra-massive WDs . . . . . . . . . . . . . . . . . . . . . . . . . . . . . . . . . . . . . .

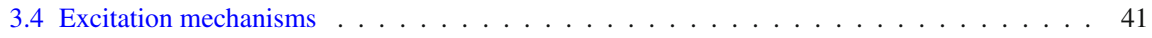

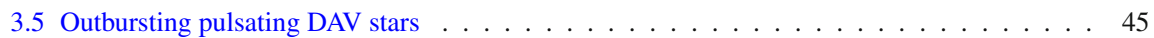

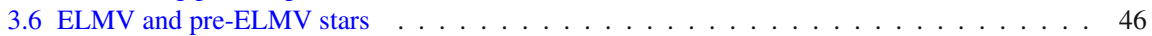

3.7 Blue large-amplitude pulsators (BLAPs) . . . . . . . . . . . . . . . . . 58

3.8 The sdA problem . . . . . . . . . . . . . . . . . . . . . . 63

3.9 WD pulsators as cosmic laboratories for fundamental physics . . . . . . . . . . . . . . 63

3.9.1 Upper bounds on the axions mass . . . . . . . . . . . . . . . . . . . . 64 64

3.9 .2 Constraints on the neutrino magnetic dipole moment . . . . . . . . . . . . . . . 68

3.9.3 Limits on the secular rate of change of the gravitational constant . . . . . . . . . . . 71

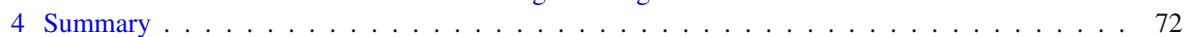

References . . . . . . . . . . . . . . . . . . . . . . . 72

\section{Introduction}

White-dwarf (WD) stars represent the final evolutionary stage of the majority of stars. Indeed, all stars with stellar masses lower than $\sim 10$ to $11 M_{\odot}$, depending on their initial metallicity (e.g., Woosley and Heger 2015), will end their lives as WDs, earth-sized electron-degenerate stellar configurations. As such, they play a unique and fundamental role for our understanding of the formation and evolution of stars, evolution of 
planetary systems, and the history of our Galaxy itself. The study of WDs thus results thus of central relevance for a vast variety of topics of modern astrophysics, ranging from the final fate of planetary systems to the characterization of dark matter (Farihi 2016; Salaris and Cassisi 2018). The present population of WDs keeps a detailed record of the early star formation in the Galaxy. Therefore, accurate WD luminosity functions can be used to infer the age, structure and evolution of the Galactic disk and the nearest open and globular clusters (Fontaine et al. 2001; Bedin et al. 2009; García-Berro et al. 2010; Bedin et al. 2015; Campos et al. 2013, 2016; García-Berro and Oswalt 2016; Kilic et al. 2017). ${ }^{1}$ In a different context, the host stars of most planetary systems, including our Sun, will evolve into WDs, and nowadays observational evidence convincingly demonstrates that numerous WDs foster remnants of planetary systems - even planetary matter, shedding light on the chemical composition of extra-solar planets (Gänsicke et al. 2012; Hollands et al. 2018). Also, WDs are found in binary systems, thus offering a test bed to explore complex stellar interactions amongst stars, including WDs exploding as type Ia supernovae (Maoz et al. 2014). In addition, WDs can be used as cosmic laboratories of extreme physics, ranging from atomic and molecular physics in strong magnetic fields, and high-density plasmas and even solid-state physics (through crystallization; Winget et al. 2009; Tremblay et al. 2019), to exotic physics, like constraining the axion mass and the possible variation of the gravitational constant (Isern et al. 1992; Córsico et al. 2012b, 2013), and also variations of the fine-structure constant (Hu et al. 2019). Last but not least, fundamental properties of WDs, either individually or collectively, like the mass distribution, core chemical composition, and cooling times are key to place constraints on the stellar evolution theory, including third dredge up and mass loss on the Asymptotic Giant Branch (AGB), the efficiency of extra-mixing during core helium burning, and nuclear reaction rates (Kunz et al. 2002; Salaris et al. 2009; Fields et al. 2016). Excellent review papers describing the evolutionary properties of WDs are those of D'Antona and Mazzitelli (1990), Koester and Chanmugam (1990), Fontaine et al. (2001), Koester (2002), Hansen and Liebert (2003), Hansen (2004), Fontaine and Brassard (2008), Winget and Kepler (2008), and Althaus et al. (2010b).

Like many stars, when relevant layers are required to transport energy through high opacity, WDs exhibit periodic brightness variations which are due to global pulsations associated with their normal modes (Ledoux and Walraven 1958; Cox 1980; Unno et al. 1989). The existence of these intrinsic luminosity variations implies that, in principle, we have available a unique window to "look" inside these stars, otherwise inaccessible by other means. The analysis of pulsations of a variety of stars has led, in the last decades, to the development of novel techniques which, taken together, are known today as asteroseismology (Aerts et al. 2010; Balona 2010; Catelan and Smith 2015). In principle, a key factor for a successful asteroseismological analysis is the number of periods visible in the star, i.e., the more periods a pulsating star exhibits, the stronger the constraints that asteroseismology could place. It must be emphasized, however, that the crucial point for a successful asteroseismological exercise is not the absolute number of modes itself, but the diversity in the eigenfunctions of these modes. Putting it in other terms: the information provided by a few periods corresponding to

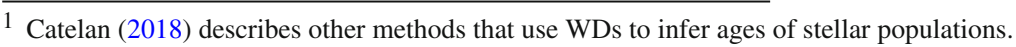


low-order modes is generally richer than the information that provides a larger set of modes with periods in the asymptotic regime (high-order modes).

Most WD stars go through at least one stage of pulsational instability during their lives, ${ }^{2}$ which turns them into multi-periodic pulsating variable stars and therefore, it is possible to analyze their internal structure employing the tools of asteroseismology (Winget 1988; Kepler and Bradley 1995; Fontaine and Brassard 2008; Winget and Kepler 2008; Althaus et al. 2010b; Vauclair 2013; Kepler and Romero 2017; Córsico 2018). Pulsations in WDs manifest themselves as periodic brightness variations in the optical and also in the ultraviolet (UV) regions of the electromagnetic spectrum. These variations are generated by global nonradial $g$ (gravity)-mode pulsations which are a subclass of spheroidal modes ${ }^{3}$ whose main restoring force is gravity through buoyancy. The pulsations are characterized by peak-to-peak amplitudes between 0.1 mmag and 0.4 mag in typical optical light curves.

An increasing number of categories of WD pulsators have been discovered since 1968. At present, there are six classes of confirmed pulsating WDs known (see Fig. 1). They are:

- The variables ZZ Ceti or DAVs - pulsating WDs with almost pure H atmospheresare the most numerous ones (Kepler and Romero 2017). They are located at low effective temperatures and high gravities $\left(10400 \mathrm{~K} \lesssim T_{\text {eff }} \lesssim 12400 \mathrm{~K}\right.$ and $7.5 \lessgtr \log g \lesssim 9.1$ ). It was the first class of pulsating WDs to be detected (Landolt 1968). Many DA WDs and so, some DAV stars, have an atmosphere polluted by the accretion of heavy elements from a debris disk (Koester et al. 2014; Wachlin et al. 2017).

- The GW Lib stars, which are accreting pulsating WDs in cataclysmic variables $\left(10500 \mathrm{~K} \lesssim T_{\text {eff }} \lesssim 16000 \mathrm{~K}\right.$ and $\left.8.3 \lesssim \log g \lesssim 8.7\right)$. They have H-dominated atmospheres, but due to accretion from a solar composition or He-enriched lowmass companion, they can have an enhanced He abundance (Szkody et al. 2010). The first object of this kind, GW Librae, was discovered by Warner and van Zyl (1998).

- The variables V777 Her or DBVs (atmospheres almost pure in He, $22400 \mathrm{~K} \lesssim$ $T_{\text {eff }} \lesssim 32000 \mathrm{~K}$ and $7.5 \lesssim \log g \lesssim 8.3$ ), the existence of which was theoretically predicted by Winget et al. (1982b) before their discovery (Winget et al. 1982a).

- The pulsating PG1159 stars or GW Vir variable stars, after the prototype of the class, PG 1159-035 (McGraw et al. 1979). This is the hottest known class of pulsating WDs and pre-WDs $\left(80000 \mathrm{~K} \lesssim T_{\text {eff }} \lesssim 180000 \mathrm{~K}\right.$ and $5.5 \lessgtr \log g \lesssim$ 7.5), constituted by variable $\mathrm{H}$-deficient, C-, O- and He-rich atmosphere WD and pre-WD stars. This group includes objects that are still surrounded by a nebulathe variable planetary nebula nuclei, designed as PNNVs - and stars that lack a nebula - called DOVs. The cause of the pulsations of these stars was unraveled by Starrfield et al. (1983) and Starrfield et al. (1984)

\footnotetext{
2 An exception are the high-field magnetic WDs that represent the $\sim 20 \%$ of the local population of WDs, and for which there is no observational evidence of variability due to pulsations.

${ }^{3}$ Spheroidal modes are characterized by $(\nabla \times \xi)_{r}=0$ and $\sigma \neq 0$, where $\xi$ is the Lagrangian displacement and $\sigma$ the pulsation frequency (Unno et al. 1989).
} 


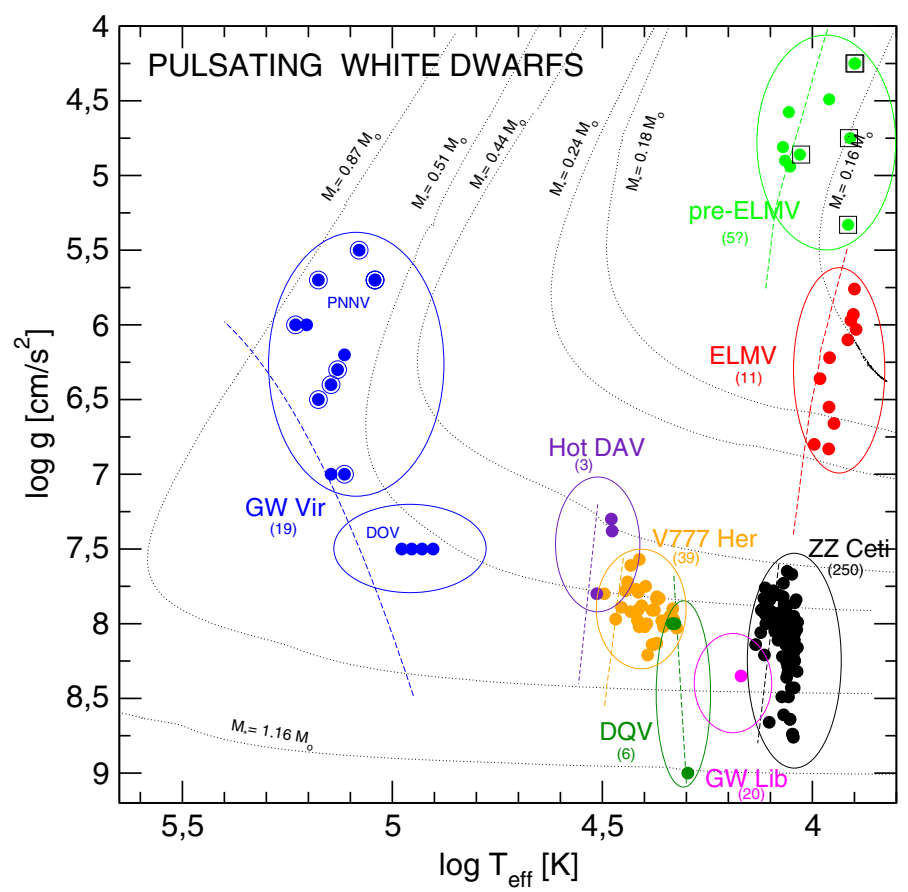

Fig. 1 Location of the different classes of confirmed and tentative pulsating WD and pre-WD stars (circles of different colors) in the $\log T_{\text {eff }}-\log g$ diagram. This figure is an update of Fig. 15 of Althaus et al. (2010b). Stars emphasized with squares surrounding the light green circles can be identified as pre-ELMV stars as well as SX Phe and/or $\delta$ Scuti stars. GW Vir stars indicated with blue circles surrounded by blue circumferences are PNNVs. In the case of GW Lib stars, only the location of the prototypical object, GW Librae, has been included (magenta dot). Two post-VLTP (Very Late Thermal Pulse) evolutionary tracks for H-deficient WDs (0.51 and $0.87 M_{\odot}$; Miller Bertolami and Althaus 2006), four evolutionary tracks of low-mass He-core H-rich WDs $\left(0.16,0.18,0.24\right.$, and $0.44 M_{\odot}$; Althaus et al. 2013), and one evolutionary track for ultra-massive H-rich WDs (1.16 $M_{\odot}$; Camisassa et al. 2019) are plotted for reference. Dashed lines indicate the theoretical blue edge of the instability domains for the different classes of pulsating WDs

- The ELMVs (Extremely Low-Mass WDs variable, $7800 \mathrm{~K} \lesssim T_{\text {eff }} \lesssim 10000 \mathrm{~K}$ and $6 \lesssim \log g \lesssim 6.8$, pure $\mathrm{H}$ atmospheres), discovered by Hermes et al. (2012).

- The pre-ELMVs $\left(8000 \mathrm{~K} \lesssim T_{\text {eff }} \lesssim 13000 \mathrm{~K}\right.$ and $\left.4 \lesssim \log g \lesssim 5\right)$, the probable precursors of ELMVs (Maxted et al. 2013).

Also, there are two additional classes of tentative WD pulsators, that need confirmation:

- The hot DQ variable WDs, or DQVs $\left(19000 \mathrm{~K} \lesssim T_{\text {eff }} \lesssim 22000 \mathrm{~K}\right.$ and $8 \lesssim$ $\log g \lesssim 9$ ). They are WDs with C- and He-rich atmospheres. The prototype of this class, SDSS J142625.71+575218.3, was discovered by Montgomery et al. (2008). We caution that the variability of some of these objects could be explained by other effects than pulsations (Williams et al. 2013).

- The so-called "hot DAVs" $\left(T_{\text {eff }} \sim 30000 \mathrm{~K}, 7.3 \lesssim \log g \lesssim 7.8\right.$; Kurtz et al. $2008,2013)$, whose existence was anticipated by the theoretical calculations of 
Shibahashi $(2005,2007)$. The pulsating nature of the variability of these stars needs to be confirmed with further observations.

In Table 1, we present a compact summary of the main pulsation characteristics of each class of pulsating WD stars. This is an update of Table 2 of Althaus et al. (2010b) (see, also, Table 13.1 of Catelan and Smith 2015). The first column corresponds to the name of each class, the second one gives the year of discovery of the first member of the group and the number of known members (in parenthesis), columns 3-8 correspond to the effective temperature, the logarithm of the surface gravity, the range of observed periods, the range of the rates of period change, the interval of amplitudes, and the surface chemical composition of each class, respectively.

Regarding the driving mechanisms involved in the excitation of the pulsations in WDs, there is a strong consensus that they correspond to thermal processes that give place to self-excited pulsations. ${ }^{4}$ The more relevant mechanisms are the $\kappa-\gamma$ mechanism, that involves an increase in the opacity of the material due to the partial ionization of the dominant chemical species (Dolez and Vauclair 1981; Winget et al. 1982b; Cox et al. 1987; Gautschy et al. 2005), and the "convective driving" mechanism (Brickhill 1991; Goldreich and Wu 1999) that acts efficiently when the outer convection zone deepens. In the case of GW Vir stars, which lack a surface convection zone due to their very-high effective temperatures, only the $\kappa-\gamma$ mechanism appears to be the one responsible for pulsations. Finally, the $\varepsilon$ mechanism due to stable nuclear burning could be able to excite short-period $g$ modes in GW Vir stars (Kawaler et al. 1986), ELMVs (Córsico and Althaus 2014b), and ZZ Ceti stars evolved from low-metallicity progenitors (Camisassa et al. 2016). Also, $g$-mode excitation by the $\varepsilon$ mechanism has been predicted for very hot H-rich pre-WDs (Maeda and Shibahashi 2014). In spite of the fact that the origin of pulsations in WDs is known to a large extent, little is known about the agent that causes the red edge of the instability strips, ${ }^{5}$ neither why many pulsating WDs — particularly DAVs — exhibit so few periods. Fortunately, this incomplete knowledge of the physics of mode excitation and damping of WD pulsations does not prevent us from advancing in asteroseismological studies based on adiabatic calculations, in which the physical agent that gives rise to the pulsations is not relevant ${ }^{6}$, but rather the value of the periods themselves, which depends sensitively on the internal structure of WDs.

The small number of detected periods in WDs does not allow for the application of any inversion technique, as in the case of the Sun (through helioseismology), for which nearly the whole structure of the star can be determined from the eigenfunctions $^{7}$. Essentially, WD asteroseismology consists in the comparison of the individual periods and period spacings observed in variable WDs with adiabatic period and period spacings computed for a set of pulsation models - the so-called forward method. Ide-

\footnotetext{
4 This, at variance with the forced pulsations such as stochastic excitation by turbulent convection, in which the modes, that are intrinsically stable, are actually excited by convective motions.

5 Although see Quirion et al. (2012) for the case of GW Vir stars and Luan and Goldreich (2018) for the case of ZZ Ceti stars.

6 The sound of the bells (their eigenfrequencies) does not depend on how the bells are rung (Baade 1992).

7 Note, however, that in many cases, the studies of solar-type pulsators seem to be limited to using the frequency separations and frequency maximum to derive the astrophysical parameters of the stars, using the so-called "scaling relations" (Lund et al. 2017).
} 


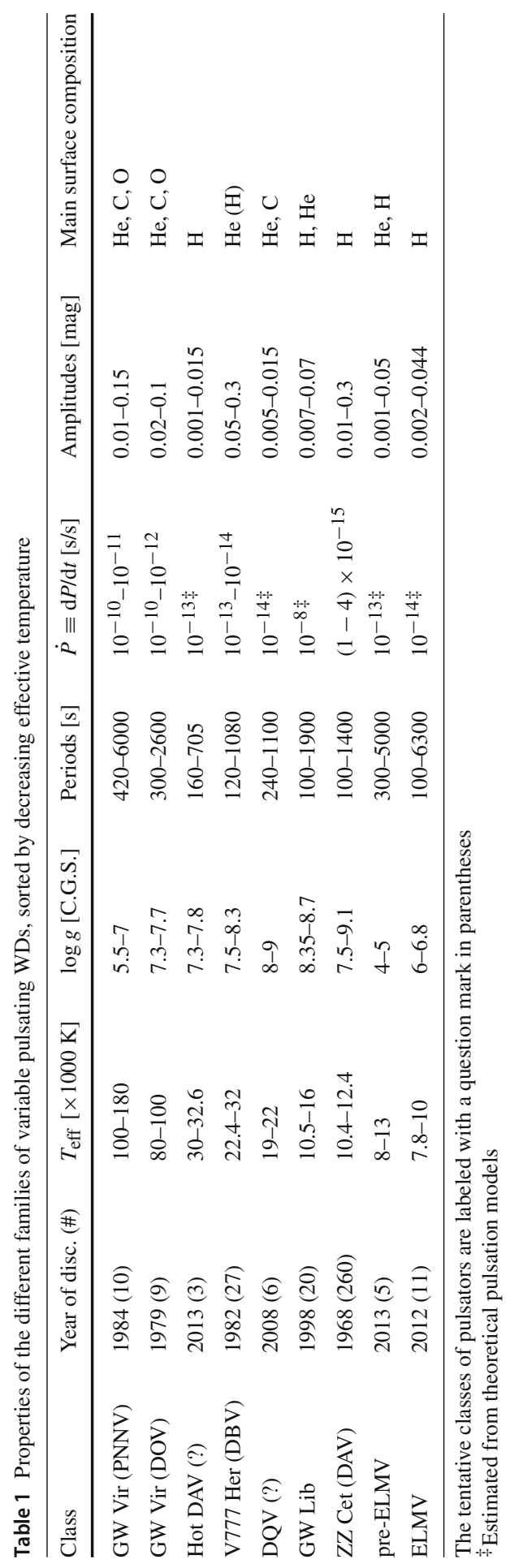


ally, when the differences between the theoretical and observed periods are small, an asteroseismological solution - the asteroseismological model- can be found. As the number of detected normal modes is usually small, this solution is not unique, and therefore external constraints such as the surface gravity and effective temperature of the star derived through spectroscopy — or distance from parallax — have to be considered to break the degeneracy of solutions. In the case of success in obtaining an asteroseismological model, one automatically has information about the internal structure, stellar mass, surface gravity, effective temperature, luminosity, radius, etc. of the star under study. With the luminosity, it is possible to infer the seismological distance, which can be compared with the distance derived through the trigonometric parallax. If it is not possible to obtain an asteroseismological model, but if it is feasible to derive a mean separation of the observed periods, it can be compared with the theoretical one, allowing us to infer the stellar mass. This last technique, however, is difficult to apply in many cases due to the simultaneous dependence of the mean period spacing on the stellar mass, the effective temperature, and the thickness of the outer envelopes of WDs. ${ }^{8}$ In connection with this, the departures of the period separation from a constant period spacing tell us about the steep variations in density caused by chemical transition regions of WDs. Another tool of WD asteroseismology is the analysis of the splitting of the frequencies, which can give clues about the nature and magnitude of rotation (angular velocity) and magnetic fields. In particular, Kawaler et al. (1999) were the first to explore the potential of the inversion methods employed in helioseismology to infer the internal rotation of GW Vir and V777 Her stars. Finally, there is the secular drift of the pulsation periods. Although the measurement of this quantity is extremely difficult, the rate of period change can give valuable information about the core chemical composition of WDs and the cooling rate.

In this review, we will not focus on the details about the asteroseismological tools applied to pulsating WDs, which can be found in Fontaine and Brassard (2008), Winget and Kepler (2008), Althaus et al. (2010b), Kepler and Romero (2017), and Giammichele et al. (2017b). Instead, we will concentrate on the advancements made in the study of pulsating WDs in the last decade.

\section{Evolutionary channels and uncertainties in progenitor evolution}

More than 60 years after its humble beginnings (Schwarzschild 1958), stellar-evolution theory is nowadays a well-established and predictive theory (Kippenhahn et al. 2012). After more than half a century of continuous development, its main predictions have been confirmed by a variety of different observational tests. This is even more true in the case of the low- and intermediate-mass stars, which are the progenitors of WD stars, where rotation and magnetic fields do not play a major role in determining the internal structure. As a consequence, we can now use the predictions of the stellarevolution theory to learn about the evolution of WD progenitors and make educated guesses about their internal structure and composition.

\footnotetext{
${ }^{8}$ However, the dependence of the period spacing on the thickness of the outer envelope of DA and DB WDs is generally weaker than its dependence upon the effective temperature and the stellar mass (Tassoul et al. 1990).
} 


\subsection{Single evolution}

In the simplest picture, WD stars are formed once winds remove most of the H-rich envelope below the critical value required to sustain a giant-like structure. For most single low- and intermediate-mass stars, this happens once the star finishes burning He in the core, and evolves to the Thermally Pulsating (TP) Asymptotic Giant Branch (AGB). On the TP-AGB phase, stars undergo intense radiation dust-driven winds as high as $\dot{M} \sim 10^{-4} M_{\odot} /$ year, where most of the envelope can be removed in less than one million years, leaving a carbon-oxygen (CO) core WD (Herwig 2005; Höfner and Olofsson 2018). While most low- and intermediate-mass stars will reach the TPAGB phase, progenitors with the lower masses might lose enough mass on the first ascent of the red giant branch (RGB) to populate the extreme horizontal branch (sdB stars) and directly evolve to the WD cooling track without reaching the AGB phase (the so-called "AGB-Manqué" stars), see Greggio and Renzini (1990) and references therein. Although a CO-core WD is the most common end state for single stars, other possibilities exist. In fact, on the one hand, the heaviest intermediate-mass stars $\left(M_{i} \gtrsim 8 M_{\odot}\right)$ might reach temperatures in the core high enough to ignite $\mathrm{C}$, enter the Super-AGB phase and end up as oxygen/neon (ONe)-core WDs (Ritossa et al. 1996; Siess 2010) $)^{9}$. On the other hand, stars with low initial masses $\left(M_{i} \lesssim 0.65 M_{\odot}\right)$, low initial metallicities and high initial He contents are able to evolve away from the main sequence in timescales shorter than a Hubble time, and might lose their H-rich envelopes already on the first RGB, leading to the formation of relatively massive He-core WDs $\left(M_{f} \gtrsim 0.4 M_{\odot}\right.$, see Ventura et al. 2001; Norris 2004; Calamida et al. 2008; Strickler et al. 2009; Bellini et al. 2013; Althaus et al. 2017, and references therein).

Although the overall picture of the evolution of single WD progenitors was well established many decades ago, some uncertainties remain about the details of the preWD evolution. Among them, convective boundary mixing remains the largest one. While extra-mixing in H-burning cores during the main sequence is relatively well constrained and calibrated (Pietrinferni et al. 2004; Ekström et al. 2012), the extent of convective boundary mixing during the He-core burning (Charpinet et al. 2011; Constantino et al. 2015) and TP-AGB phases is somewhat uncertain. The lack of a complete understanding of convective boundary mixing, together with the uncertainties in the intensity of winds during the TP-AGB phase, lead to uncertainties in the Initial-Final Mass Relationship (IFMR) of stellar evolution models (see Salaris et al. 2009 for a detailed study of the uncertainties). Among other things, this implies that IFMRs cannot be reliably predicted by current stellar evolution models, but instead semi-empirical IFMRs must be used (together with other observables) to calibrate macrophysics processes in stars (Miller Bertolami 2016). In fact, the very existence of a tight IFMR is not supported by semi-empirical determinations of the IFMR, which gives a significant scatter in the mass of the WD for a given initial mass (Casewell et al. 2009; Salaris et al. 2009; Cummings et al. 2018). In addition, as most semi-empirical

\footnotetext{
${ }^{9}$ Gänsicke et al. (2010) discovered two WDs exposing dredged-up, O-rich core material that could have been produced in the interior of a Super-AGB star. Recently, Kepler et al. (2016a) identified a WD having an O-dominated atmosphere with traces of $\mathrm{Ne}$ and $\mathrm{Mg}$, that could be the bare core of a Super-AGB star. Finally, another O- and Ne-rich WD but with a very low mass was discovered by Vennes et al. (2017).
} 
determinations of the IFMR have been performed for solar-like metallicities, we currently do not know how IFMRs depend on metallicity or He content. Uncertainties in the IFMRs and convective boundary mixing processes of the models impact the chemical profiles of WD stars of a given mass (see later).

In the simple scenario discussed above, stellar evolution theory usually predicts the formation of WDs with pure $\mathrm{H}$ atmospheres and with a total $\mathrm{H}$ content of about $M_{\mathrm{H}} \sim 10^{-3}-10^{-5} M_{\odot}$. About $80 \%$ of the spectroscopically identified WDs are characterized by H-rich atmospheres. The remaining 20\% of WDs are characterized by He-dominated atmospheres (spectral types PG1159, DO, DB, DQ, DZ, DC; see Althaus et al. 2010b). In addition, systematic spectroscopic and asteroseismological studies of DA stars indicate that between $15 \%$ and $20 \%$ of DAs have thin $\mathrm{H}$ envelopes with $M_{\mathrm{H}} \lesssim 10^{-6} M_{\odot}$ (see Tremblay and Bergeron 2008; Castanheira and Kepler 2009; Romero et al. 2012). Both WDs with He-dominated or thin H envelopes cannot be explained by the simple picture presented above and more complex evolutionary scenarios have been developed to explain their existence. Besides the binary-evolution channels to be discussed in the next section, some single-evolution scenarios predict the formation of both He-dominated atmospheres and thin $\mathrm{H}$ envelopes.

D'Antona and Mazzitelli (1990) reviewed several evolutionary channels for the formation of WDs with low $\mathrm{H}$ contents. In particular, thermal pulses during the postAGB phase lead in a very natural way to the formation of WDs with low $\mathrm{H}$ contents. Iben (1984) showed that depending on the timing of the departure from the AGB phase, a last thermal pulse can happen during the post-AGB evolution. If a late thermal pulse develops when the post-AGB star is already entering the WD cooling track, then the $\mathrm{H}$-rich envelope will be ingested by the He-shell flash convective zone, where $\mathrm{H}$ will be burnt in the hot interior of the star (Iben and MacDonald 1995; Herwig et al. 1999). This scenario was named a Very Late Thermal Pulse (VLTP) by Blöcker (2001). Although numerical simulations never predict the burning of the complete $\mathrm{H}$ content of the star, it has been argued that whatever the traces of $\mathrm{H}$ that may be left by the VLTP, they will very likely be peeled off by mass loss during the subsequent giant phase (Werner and Herwig 2006). While this is a possibility, it has to be noted that, once the star is back on its giant phase, $\mathrm{H}$ is diluted in the more massive convective envelope of the born again AGB star $\left(M_{\text {env }} \sim 10^{-3} M_{\star}\right)$. The star would need to lose all that mass to get rid of its whole $\mathrm{H}$ content. If this is the case, then this scenario will produce a WD with a H-deficient atmosphere. It should be noted, however, that the study of Miller Bertolami and Althaus (2007) suggests that the amount of H burned might depend on the total mass of $\mathrm{H}$ remaining in the star at the moment of the VLTP, and VLTPs in low-mass stars might only burn a small fraction of the total H content of the star. Consequently, in this case, a VLTP will lead to the formation of DA WDs with very low $\mathrm{H}$ contents $\left(M_{\mathrm{H}} \lesssim 10^{-7} M_{\odot}\right.$, see Miller Bertolami et al. 2017). If a thermal pulse happens during the horizontal evolution of the post-AGB star in the HR diagram, a scenario termed Late Thermal Pulse (LTP) by Blöcker (2001) occurs. Then, the H-rich envelope is not burned but diluted by the deepening of the convective envelope, once the star evolves back to the AGB after the LTP. As shown by Althaus et al. (2005), the $\mathrm{H}$ diluted into the deeper parts of the envelope is later burned as the H-deficient central star contracts again to the WD cooling track, leading to the formation of WDs with a low $\mathrm{H}$ content $\left(M_{\mathrm{H}} \lesssim 10^{-6}-10^{-7} M_{\odot}\right)$. Finally, D' Antona 
and Mazzitelli (1990) mentioned that the diffusion-induced nova studied by Iben and MacDonald (1986) was also a possible channel for the formation of DA WDs with low H contents, but the later work by Miller Bertolami et al. (2011) showed that this scenario does not lead to a significant reduction in the $\mathrm{H}$ content of the future WD.

A few final words of caution on some widespread misconceptions about the relevance of stellar winds for the formation of WDs with low $\mathrm{H}$ contents are in order. Although it has been known for many decades that the H content of the future WD cannot be arbitrarily reduced by winds during the AGB or post-AGB H-burning phases, (see Schoenberner 1987, and references therein), some confusion has arisen in recent works. As already shown by Paczyński (1971), H-burning post-AGB models have a very tight relationship between the effective temperature and the envelope mass of the post-AGB object. As a consequence, and as long as the envelope can be considered in thermal equilibrium, the location of a H-burning post-AGB remnant on the HR diagram is independent of the mass-loss history and only dependent on the value of the envelope mass. This implies that an enhancement in the post-AGB winds does speed up the post-AGB evolution but does not reduce the final $\mathrm{H}$ content of the WD; see Althaus et al. (2015), Miller Bertolami et al. (2017). Note, however, that more intense winds in a He-burning post-AGB object can indeed reduce the final $\mathrm{H}$ content of the WD significantly. Yet, due to the relatively short duration of the He-burning phase of AGB and post-AGB models, the star has to undergo a final He flash at or very close to the departure from the AGB, and this is only relevant within the late thermal pulse scenario discussed before. A similar situation holds for winds during the AGB and the departure from the AGB phase. An enhancement of AGB winds leads to a shortening of the AGB phase. With less time for the $\mathrm{H}$-free core to grow during the thermal pulses, enhancing the winds on the AGB leads to a smaller final mass for the same initial mass. Due to the tight $M_{\mathrm{WD}}-M_{\mathrm{H}}^{\mathrm{WD}}$ relation, this implies that more intense winds on the AGB lead to the formation of WDs with higher H contents. This is true also when looked at the same value of the WD mass $\left(M_{\mathrm{WD}}\right)$. Models of the same final mass but shorter AGB lifetimes are less compact and luminous and have, consequently, larger post-AGB envelope masses (Blöcker 1995; Miller Bertolami 2016). Consequently, more intense winds on the AGB usually lead to larger $\mathrm{H}$ envelopes for WDs. In closing, nowadays, the LTP and VLTP scenarios are the best explanations for the formation of WDs with low $\mathrm{H}$ contents in the context of single stellar evolution.

The internal chemical constitution of WDs is a crucial issue for the determination of the pulsational properties of these stars. In Fig. 2, we show the chemical structure of a template DA WD model with $M_{\star}=0.56 M_{\odot}, T_{\text {eff }} \sim 12000 \mathrm{~K}$, and $\log \left(M_{\mathrm{H}} / M_{\star}\right) \sim-4$, resulting from the evolution of a single $1 M_{\odot}$ progenitor from the ZAMS to the WD phase. We display the mass fraction of ${ }^{16} \mathrm{O},{ }^{12} \mathrm{C},{ }^{4} \mathrm{He}$, and ${ }^{1} \mathrm{H}$, as a function of the outer mass coordinate. Note that this coordinate strongly emphasizes the external part of the WD star. We include a short account of the origin and the uncertainties playing a role at each part of the chemical structure. The extreme sensitivity of the pulsation properties of WDs to the details of the chemical structure links the asteroseismological inferences of pulsating WDs to the physical processes that take place during the progenitor's evolution. However, it has not been until recently that the impact of the current uncertainties in stellar evolution, both concerning the modeling of physical processes and input physics of WD progenitors, have begun to be 


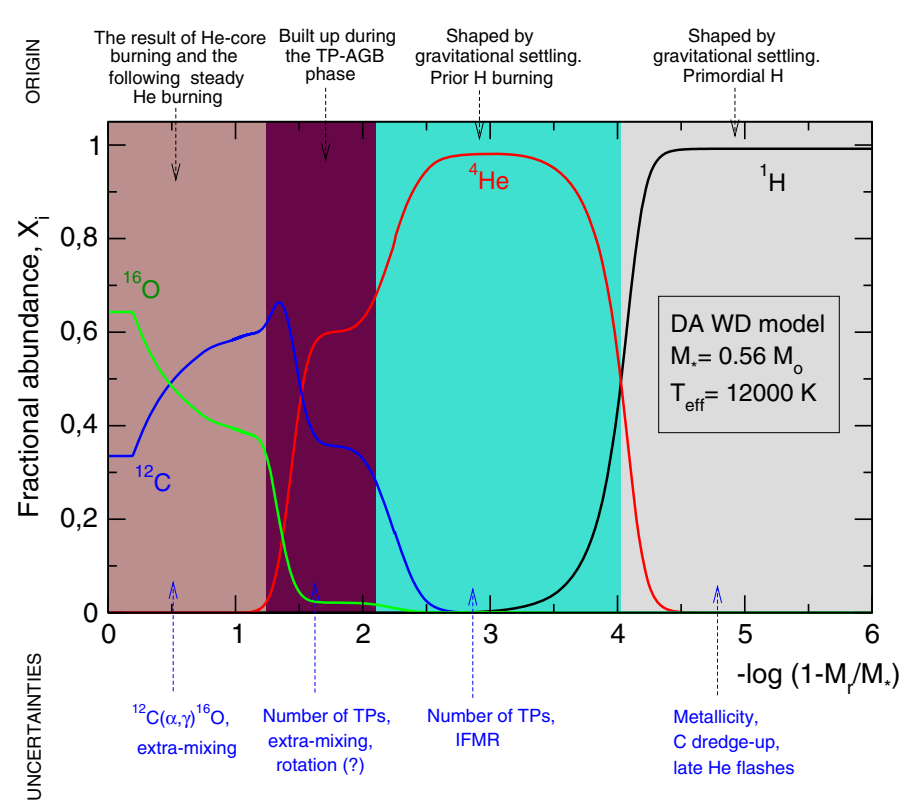

Fig. 2 Internal chemical structure of a typical DA WD model with $M_{\star}=0.56 M_{\odot}, T_{\text {eff }} \sim 12000 \mathrm{~K}$, and $\mathrm{H}$ envelope thickness of $\log \left(M_{\mathrm{H}} / M_{\star}\right) \sim-4$, resulting from the complete evolution of a single $1 M_{\odot}$ progenitor from the ZAMS to the WD stage at the ZZ Ceti instability domain. Plotted is the mass fraction $\left(X_{i}\right)$ of ${ }^{16} \mathrm{O}$ (green line), ${ }^{12} \mathrm{C}$ (blue line), ${ }^{4} \mathrm{He}$ (red line), and ${ }^{1} \mathrm{H}$ (black line), in terms of the outer mass coordinate. We include in the plot a brief explanation about the origin (up) and the uncertainties (down) of each part of the internal chemical structure, emphasized with different colors

assessed in asteroseismological fits. In this regard, De Gerónimo et al. (2017) explored for the first time the impact of the occurrence of TPs on the AGB in WD progenitors, the uncertainty in the ${ }^{12} \mathrm{C}(\alpha, \gamma){ }^{16} \mathrm{O}$ cross section, and the occurrence of extra mixing episodes during core He burning on the expected period spectrum of ZZ Ceti stars ${ }^{10}$. In this connection, the mixing and burning processes that take place along the thermally pulsing AGB phase build the chemical stratification of the outer layers of the CO-core of the emerging WD (Althaus et al. 2010a), in particular the He- and C-rich inter-shell region that is formed during this stage as a result of the short-lived He flash convection zone induced by the peak flash (see Fig. 2). The mass of this inter-shell, as well as the mass of the total He content of the WD, depends on both the occurrence of overshooting (OV) during the He flash and the number of thermal pulses. In turn, the number of thermal pulses is determined by the initial mass, chemical composition and by the poorly constrained efficiency of mass loss (Karakas and Lattanzio 2014). As we will discuss, the shape of the chemical profiles left by evolution during the thermally pulsing AGB phase markedly impacts the $g$-mode pulsational periods, as shown in De Gerónimo et al. (2017), who also concluded that the occurrence or not of the TP-AGB phase during the evolution of the WD progenitor constitutes a relevant issue that has to be taken into account in seismological period fits of these stars. As

\footnotetext{
${ }_{10}$ Previous efforts to constrain the ${ }^{12} \mathrm{C}(\alpha, \gamma){ }^{16} \mathrm{O}$ reaction rate using WD asteroseismology have been done using DBVs (see, e.g., Metcalfe 2003, and references therein).
} 
mentioned, despite the fact that the occurrence of the TP-AGB phase is expected for most of single WD progenitors, it is not discarded that some WDs could have evolved from progenitor stars that avoided this phase. In fact, it is well known that low-mass He-burning stars located at the extreme horizontal branch, and thus characterized by extremely thin H envelopes (Faulkner 1972), evolve directly to the WD stage, avoiding the AGB (the AGB-Manqué and post early AGB stars; see Caloi 1989; Brocato et al. 1990; Greggio and Renzini 1990). In line with this, recent evidence suggests that most He-rich stars of the globular cluster NGC2808 do not reach the AGB phase, evolving directly to the WD state after the end of the He core burning (Marino et al. 2017). In addition, departure from the AGB before reaching the TP-AGB phase as a result of mass transfer by binary interaction (Han et al. 2000) or envelope ejection by the swallowing of a planet or a very-low-mass companion (De Marco and Soker 2002) is also possible. Accordingly, major differences in the chemical structure of the outermost layers of WDs should be expected depending on whether the progenitor stars evolved through the thermal pulses on the AGB or not, with consequences for the expected pulsational properties of pulsating WDs.

\subsection{Binary evolution}

In a binary system, if the binary orbit is wide enough, the individual stars are not affected by the presence of a companion; so, single stellar evolution theory is enough to describe their evolution (see previous section). However, if the stars become close, they can interact, with severe consequences for their evolution. Interaction can happen by tidal forces, by stellar winds, or by mass transfer and accretion. If either star fills its Roche lobe, then gas flows from the outer layers of that star into the Roche lobe of the companion star. Some or all of this gas may be captured by the companion star, so that mass transfer occurs. When the star filling the Roche lobe is a giant that has a convective envelope, and when the donor star is significantly more massive than its companion, the transferred mass may not be accreted by the companion, leading to the formation of a common envelope (CE) surrounding both stars. The outcome of CE evolution is still not fully understood (Ivanova et al. 2013; Nandez and Ivanova 2016), but possible outcomes involve the formation of a closer binary or a stellar merger. If the system is left in a very close binary configuration, then the radiation of gravitational waves will further shrink the orbits leading to a stellar merger.

The stable Roche lobe overflow channel (RLOF) is of particular interest for the formation of He-core WDs. In fact, due to the very long lifetimes on the main sequence, low-mass He-core WDs cannot be formed within single stellar evolution. For this reason, the most accepted channel for the formation of these stars involves one, or more, phases of mass transfer. In particular, if a low-mass star fills its Roche lobe during the RGB, the H-rich envelope can be stripped before the He-core becomes massive (and hot) enough to ignite He. As a consequence, once all but a thin H-rich envelope is stripped from the star, it cannot support its giant configuration anymore, and contracts to become a He-core WD (Althaus et al. 2013; Istrate et al. 2016b).

The coalescence of two stars within a common envelope evolution or in a tight close binary system after a common envelope event has been proposed to explain the 
properties of isolated WD stars. In particular, the merger of two WDs may give rise to Type Ia Supernovae and to a variety of objects such as Hot-subdwarf ( $\mathrm{sdO} / \mathrm{sdB}$ spectral types) stars and R CrB stars that will finally evolve into WDs (Dan et al. 2014). The WDs formed by these channels might harbor $\mathrm{CO}$ or ONe cores with either H-rich or H-deficient atmospheres.

\section{Asteroseismology of pulsating WDs and pre-WDs}

\subsection{Recent observational achievements}

As shown in Table 1 and Fig. 1, at present there are eight families of pulsating WDs and pre-WDs, although two of those categories (hot DAVs and DQVs) need to be confirmed as such. Here, we describe the new observational findings in the field of these pulsating degenerate stars.

In the last decade, there have been numerous discoveries of pulsating WD stars, both from the ground and from space. Ground-based observations, mainly with the spectral observations of the Sloan Digital Sky Survey (SDSS; York et al. 2000), have increased the number of known WDs by a factor of 15 (Kleinman et al. 2013; Kepler et al. 2016b, 2019; Kepler and Romero 2017; Gentile Fusillo et al. 2019) and the number of pulsators by a factor of 4, starting with Mukadam et al. (2004), Mullally et al. (2005), Castanheira et al. (2006), Voss et al. (2007), Nitta et al. (2009), Castanheira et al. (2013). In Tables 2, 3, 4, 5 we present the list of ZZ Ceti stars known at the time of writing this review (March 2019), along with their effective temperatures, surface gravities, and magnitudes. The determinations of $\log g$ and $T_{\text {eff }}$ from the spectra, when available, were taken from the literature, mainly Gianninas et al. (2011) and Kepler et al. (2019), with 3D corrections (following Tremblay et al. 2013b) applied.

Very recently, 36 new DAVs have been discovered by Rowan et al. (2019), but for most of them, their spectroscopically determined gravities and effective temperatures are not available yet. When only colors were available, they were used as in Kepler et al. (2019). Tucker et al. (2018) discovered a new ZZ Ceti star, WD2246069, using gPhoton, a timetagged database of GALEX photon events and associated software package. For ZZ Cetis, the discovery of the rare ultra-massive pulsators, which started with BPM 37093 with $M_{\star} \sim 1.1 M_{\odot}$ (Kanaan et al. 1992), includes GD 518, with $M_{\star} \sim 1.24 M_{\odot}$ (Hermes et al. 2013a), and SDSS J084021.23+522217.4 (Curd et al. 2017 ), with $M_{\star} \sim 1.16 M_{\odot}$, opened the study of crystallized WDs. In addition, WD $\mathrm{J} 212402.03-600100.0$ is another possible ultra-massive DAV $\operatorname{star}\left(M_{\star} \sim 1.16 M_{\odot}\right.$; see Rowan et al. 2019). New theoretical work about ultra-massive ZZ Cetis will be introduced in Sect. 3.3. On the other hand, the 3D convection studies of Tremblay et al. (2013a, 2015) have converged the determination of temperatures and gravities using different mixing length models, which had plagued prior studies of the location of the ZZ Ceti instability strip.

Despite numerous discoveries of pulsating WDs_-and notwithstanding several successful asteroseismological studies carried out in the last decade, see Sect. 3.2- the discovery of new pulsators has not led to asteroseismological solutions for most of them, because the available discovery data in general cover only a few hours of time 
Table 2 ZZ Ceti stars and their effective temperatures, surface gravities and magnitudes

\begin{tabular}{|c|c|c|c|}
\hline Name & $T_{\text {eff }}(\mathrm{K})$ & $\log g$ & Magnitude \\
\hline SDSS J000006.75-004654.0 & 10620 & 8.18 & $18.8(\mathrm{~g})$ \\
\hline SDSS J001836.11+003151.1 & 11530 & 8.04 & $17.4(\mathrm{~g})$ \\
\hline МСТ 0016-2553 & 11060 & 8.06 & $15.9(\mathrm{GG})$ \\
\hline HE $0031-5525$ & 11662 & 7.71 & $15.7(\mathrm{~g})$ \\
\hline G $132-12$ & 12480 & 8.00 & $16.3(\mathrm{~g})$ \\
\hline SDSS J004345.78+005549.9 & 12130 & 8.14 & $18.7(\mathrm{~g})$ \\
\hline LAMOST J004628.31+343319.9 & 11681 & 7.53 & $16.3(\mathrm{~g})$ \\
\hline SDSS J004855.17+152148.7 & 11280 & 8.17 & $18.7(\mathrm{~g})$ \\
\hline SDSS J005208.42-005134.6 & 12300 & 8.46 & $17.7(\mathrm{~g})$ \\
\hline SDSS J010207.17-003259.4 & 10850 & 8.18 & $18.0(\mathrm{~g})$ \\
\hline EPIC 220274129 & 11810 & 8.03 & $16.7(\mathrm{~g})$ \\
\hline LAMOST J010302.46+433756.2 & 11750 & 7.89 & $18.3(\mathrm{~g})$ \\
\hline BPM 30551 & 11240 & 8.16 & $15.4(\mathrm{~g})$ \\
\hline SDSS J011100.63+001807.2 & 11490 & 8.08 & $18.8(\mathrm{~g})$ \\
\hline SDSS J011123.89+000935.3 & 12321 & 7.50 & $17.8(\mathrm{~g})$ \\
\hline SDSS J012234.68+003025.8 & 11650 & 7.94 & $16.8(\mathrm{~g})$ \\
\hline SDSS J012950.44-101842.0 & 12043 & 8.03 & $18.4(\mathrm{~g})$ \\
\hline LAMOST J013033.90+273757.9 & 14127 & 7.69 & $18.5(\mathrm{~g})$ \\
\hline SDSS J013440.94-010902.3 & 10260 & 7.82 & $18.1(\mathrm{~g})$ \\
\hline Ross 548 & 12300 & 8.03 & $14.3(\mathrm{~g})$ \\
\hline МCT 0145-2211 & 11850 & 8.15 & $14.9(\mathrm{GG})$ \\
\hline SDSS J020351.28+004025.1 & 10794 & 8.17 & $19.4(\mathrm{~g})$ \\
\hline HS $0210+3302$ & 12176 & 7.38 & $16.7(\mathrm{~g})$ \\
\hline SDSS J021406.78-082318.4 & 11580 & 7.86 & $17.9(\mathrm{~g})$ \\
\hline HS $0235+0655$ & 11008 & 7.56 & $16.5(\mathrm{~g})$ \\
\hline SDSS J024922.35-010006.7 & 11030 & 8.19 & $18.8(\mathrm{~g})$ \\
\hline KUV 02464+3239 & 11620 & 8.13 & $16.0(\mathrm{~g})$ \\
\hline SDSS J030153.81+054020.0 & 11139 & 8.02 & $18.1(\mathrm{~g})$ \\
\hline SDSS J030325.22-080834.9 & 11260 & 8.40 & $18.8(\mathrm{~g})$ \\
\hline SDSS J031847.09+003029.9 & 11150 & 8.18 & $17.8(\mathrm{~g})$ \\
\hline SDSS J033236.61-004918. & 10940 & 8.05 & $18.2(\mathrm{~g})$ \\
\hline BPM 31594 & 11500 & 8.05 & $15.1(\mathrm{GG})$ \\
\hline KUV $03442+0719$ & 10870 & 7.78 & $16.6(\mathrm{~g})$ \\
\hline HE $0344-1207$ & 11497 & 7.91 & $16.0(\mathrm{~g})$ \\
\hline SDSS J034939.35+103649.9 & 11896 & 8.21 & $16.6(\mathrm{~g})$ \\
\hline EPIC 210377280 & 11590 & 7.94 & $18.5(\mathrm{~g})$ \\
\hline HL Tau76 & 11470 & 7.92 & $15.0(\mathrm{~g})$ \\
\hline G $38-29$ & 11160 & 7.89 & $15.6(\mathrm{~g})$ \\
\hline G $191-16$ & 11440 & 8.04 & $15.9(\mathrm{~g})$ \\
\hline LP $119-10$ & 11342 & 8.085 & $15.2(\mathrm{~g})$ \\
\hline
\end{tabular}


Table 2 continued

\begin{tabular}{llll}
\hline Name & $T_{\text {eff }}(\mathrm{K})$ & $\log g$ & Magnitude \\
\hline HS 0507+0435 & 12010 & 8.19 & $15.3(\mathrm{~g})$ \\
GD 66 & 12210 & 8.10 & $15.5(\mathrm{~g})$ \\
HE 0532-5605 & 11510 & 8.42 & $15.9(\mathrm{GG})$ \\
LAMOST J062159.49+252335.9 & 11728 & 8.25 & $17.5(\mathrm{~g})$ \\
HS 0733+4119 & 11049 & 8.01 & $15.8(\mathrm{~g})$ \\
SDSS J075617.54+202010.2 & 11830 & 8.13 & $18.3(\mathrm{~g})$ \\
SDSS J081531.75+443710.3 & 11840 & 8.21 & $19.3(\mathrm{~g})$ \\
SDSS J081828.98+313153.0 & 11820 & 8.13 & $17.4(\mathrm{~g})$ \\
SDSS J082429.01+172345.4 & 11433 & 8.21 & $18.3(\mathrm{~g})$ \\
SDSS J082518.86+032927.8 & 12120 & 8.15 & $17.5(\mathrm{~g})$ \\
SDSS J082547.00+411900.0 & 11510 & 8.37 & $18.5(\mathrm{~g})$ \\
SDSS J083203.98+142942.3 & 11643 & 7.99 & $18.9(\mathrm{~g})$ \\
KUV 08368+4026 & 12010 & 8.13 & $15.6(\mathrm{~g})$ \\
SDSS J084054.14+145709.0 & 10862 & 7.89 & $18.3(\mathrm{~g})$ \\
SDSS J084021.23+522217.4 & 12160 & 8.93 & $18.2(\mathrm{~g})$ \\
SDSS J084220.73+370701.7 & 11620 & 7.88 & $18.8(\mathrm{~g})$ \\
SDSS J084314.05+043131.6 & 11220 & 8.09 & $17.8(\mathrm{~g})$ \\
SDSS J084746.82+451006.3 & 11690 & 8.12 & $18.3(\mathrm{~g})$ \\
SDSS J085128.17+060551.1 & 11300 & 8.05 & $16.8(\mathrm{~g})$ \\
SDSS J085325.55+000514.2 & 11950 & 8.15 & $18.2(\mathrm{~g})$ \\
SDSS J085507.29+063540.9 & 10970 & 8.22 & $17.2(\mathrm{~g})$ \\
SDSS J085648.33+185804.9 & 11896 & 8.09 & $18.9(\mathrm{~g})$ \\
SDSS J090041.08+190714.3 & 11849 & 8.05 & $17.6(\mathrm{~g})$ \\
GD 99 & 12110 & 8.20 & $14.5(\mathrm{~g})$ \\
\hline The $T$ & 1109 & \\
\hline
\end{tabular}

The $T_{\text {eff }}$ and $\log g$ values have been corrected to $3 \mathrm{D}$ model atmosphere values. The letter in parentheses in the fourth column corresponds to the filter of the magnitude. Specifically, "g" is SDSS g magnitude and "GG" is Gaia G magnitude

series photometry, which allows only for the determination of the dominant period, or worse, just the beat period. The need of detecting a large number of pulsation periods to allow the determination of the internal structure of stars can be traced back to Legendre (1806) and Gauss (1809), who demonstrated that the best way to find an unknown parameter is to minimize the sum of the square of the residuals. Laplace (1810) presented the generalization of least squares fits for multiple parameters and defined the uncertainty as $\sigma^{2}=\frac{S}{N-k}=\frac{\sum_{i=1}^{N}\left(P_{i}^{\mathrm{o}}-P_{i}\right)^{2}}{N-k}$, where $N$ is the number of measurements and $k$ the number of fit parameters; so if $k \geq N$, the uncertainty is infinite. $S$ decreases when $k$ increases, but when a term is added in a fit, it has to be tested if the decrease in $S$ is significant. The confidence level at which we can rule out the null hypothesis can be calculated through the Fisher $F$-distribution, as shown for example by Pringle (1975). However, in some cases, it is possible to constrain the structure of a pulsating WD with a few observed periods, i.e., when the number of 
Table 3 ZZ Ceti stars (continuation of Table 2)

\begin{tabular}{|c|c|c|c|}
\hline Name & $T_{\text {eff }}(\mathrm{K})$ & $\log g$ & Magnitude \\
\hline SDSS J090231.76+183554.9 & 11191 & 7.89 & $19.4(\mathrm{~g})$ \\
\hline SDSS J090624.26-002428.2 & 11260 & 8.07 & $17.7(\mathrm{~g})$ \\
\hline SDSS J091118.42+031045.1 & 11630 & 8.14 & $18.4(\mathrm{~g})$ \\
\hline SDSS J091312.74+403628.7 & 11850 & 8.09 & $17.6(\mathrm{~g})$ \\
\hline SDSS J091635.07+385546.2 & 11320 & 8.04 & $16.6(\mathrm{~g})$ \\
\hline SDSS J091731.00+092638.1 & 11340 & 8.09 & $18.1(\mathrm{~g})$ \\
\hline SDSS J092329.81+012020.0 & 11190 & 8.38 & $18.3(\mathrm{~g})$ \\
\hline G 117-B15A & 12420 & 8.12 & $15.5(\mathrm{~g})$ \\
\hline SDSS J092511.60+050932.4 & 10830 & 8.21 & $15.2(\mathrm{~g})$ \\
\hline SDSS J093944.89+560940.2 & 11690 & 8.29 & $18.7(\mathrm{~g})$ \\
\hline SDSS J094000.27+005207.1 & 10590 & 8.34 & $18.1(\mathrm{~g})$ \\
\hline SDSS J094213.13+573342.5 & 11360 & 8.12 & $17.4(\mathrm{~g})$ \\
\hline SDSS J094917.04-000023.6 & 11130 & 8.21 & $18.8(\mathrm{~g})$ \\
\hline HS $0951+1312$ & 12010 & 8.05 & $16.5(\mathrm{~g})$ \\
\hline HS $0952+1816$ & 11390 & 8.11 & $16.3(\mathrm{~g})$ \\
\hline SDSS J095833.13+013049.3 & 11730 & 8.08 & $16.7(\mathrm{~g})$ \\
\hline SDSS J095936.96+023828.4 & 11830 & 8.06 & $18.1(\mathrm{~g})$ \\
\hline SDSS J100238.58+581835.9 & 11440 & 8.11 & $18.3(\mathrm{~g})$ \\
\hline SDSS J100718.26+524519.8 & 11390 & 8.12 & $18.9(\mathrm{~g})$ \\
\hline SDSS J101519.65+595430.5 & 11440 & 8.06 & $18.0(\mathrm{~g})$ \\
\hline SDSS J101540.14+234047.4 & 11320 & 8.44 & $18.6(\mathrm{~g})$ \\
\hline SDSS J101548.01+030648.4 & 11630 & 8.12 & $15.7(\mathrm{~g})$ \\
\hline HS $1039+4112$ & 11730 & 8.12 & $16.1(\mathrm{~g})$ \\
\hline SDSS J104358.59+060320.9 & 11173 & 8.19 & $18.7(\mathrm{~g})$ \\
\hline WD $1047+335$ & 11310 & 8.09 & $16.5(\mathrm{~g})$ \\
\hline SDSS J105449.87+530759.1 & 10960 & 7.96 & $17.9(\mathrm{~g})$ \\
\hline SDSS J105612.32-000621.7 & 11130 & 7.91 & $17.5(\mathrm{~g})$ \\
\hline SDSS J110525.70-161328.3 & 11857 & 8.06 & $17.5(\mathrm{~g})$ \\
\hline SDSS J110623.40+011520.8 & 10920 & 7.90 & $18.4(\mathrm{~g})$ \\
\hline GD 133 & 12430 & 8.10 & $14.6(\mathrm{~g})$ \\
\hline SDSS J112221.10+035822.4 & 11030 & 7.91 & $18.2(\mathrm{~g})$ \\
\hline SDSS J112542.84+034506.3 & 11600 & 7.95 & $18.1(\mathrm{~g})$ \\
\hline SDSS J111710.54-125540.9 & 11302 & 8.29 & $19.6(\mathrm{~g})$ \\
\hline EC 11266-2217 & 12010 & 8.08 & $16.4(\mathrm{~g})$ \\
\hline SDSS J113604.01-013658.1 & 11780 & 8.05 & $17.8(\mathrm{~g})$ \\
\hline SDSS J113655.17+040952.6 & 12330 & 7.99 & $17.0(\mathrm{~g})$ \\
\hline KUV $11370+4222$ & 11940 & 8.17 & $16.5(\mathrm{~g})$ \\
\hline PG $1149+057$ & 11060 & 8.06 & $15.0(\mathrm{~g})$ \\
\hline EC $11507-1519$ & 12440 & 8.20 & $16.0(\mathrm{~g})$ \\
\hline SDSS J115707.43+055303.6 & 11040 & 8.04 & $17.6(\mathrm{~g})$ \\
\hline
\end{tabular}


Table 3 continued

\begin{tabular}{llll}
\hline Name & $T_{\text {eff }}(\mathrm{K})$ & $\log g$ & Magnitude \\
\hline SDSS J120054.55-025107.0 & 11970 & 8.24 & $18.2(\mathrm{~g})$ \\
G 255-2 & 11440 & 8.14 & $16.0(\mathrm{~g})$ \\
SDSS J121628.55+092246.4 & 11240 & 8.25 & $18.6(\mathrm{~g})$ \\
WD J1218+0042 & 11170 & 8.06 & $18.5(\mathrm{~g})$ \\
SDSS J122229.57-024332.5 & 11380 & 8.19 & $16.7(\mathrm{~g})$ \\
BPM 37093 & 11620 & 8.69 & $13.8(\mathrm{GG})$ \\
SDSS 124949.36+304828.1 & 12127 & 8.299 & $18.0(\mathrm{~g})$ \\
HS 1249+0426 & 12160 & 8.21 & $16.0(\mathrm{~g})$ \\
SDSS J125535.41+021116.0 & 11580 & 8.15 & $19.1(\mathrm{~g})$ \\
SDSS J125710.50+012422.9 & 11490 & 8.30 & $18.6(\mathrm{~g})$ \\
HE 1258+0123 & 11420 & 8.02 & $16.4(\mathrm{~g})$ \\
GD 154 & 11120 & 8.07 & $15.3(\mathrm{~g})$ \\
WD 1310-0159 & 10940 & 7.76 & $17.7(\mathrm{~g})$ \\
SDSS J132350.28+010304.2 & 11380 & 8.45 & $18.5(\mathrm{~g})$ \\
WD J1337+0104 & 11460 & 8.64 & $18.6(\mathrm{~g})$ \\
SDSS J133831.74-002328.0 & 11900 & 8.07 & $17.1(\mathrm{~g})$ \\
EPIC 229227292 & 11190 & 8.02 & $16.7(\mathrm{~g})$ \\
SDSS J134550.93-005536.5 & 11760 & 8.10 & $16.7(\mathrm{~g})$ \\
LP 133-144 & 12150 & 7.97 & $15.7(\mathrm{~g})$ \\
G 238-53 & 12130 & 7.97 & $15.5(\mathrm{~g})$ \\
SDSS J135459.89+010819.3 & 11650 & 8.03 & $16.4(\mathrm{~g})$ \\
SDSS J135531.03+545404.5 & 11480 & 7.93 & $18.6(\mathrm{~g})$ \\
EC 14012-1446 & 12020 & 8.18 & $15.7(\mathrm{~g})$ \\
SDSS J140859.46+044554.7 & 10920 & 7.99 & $17.9(\mathrm{~g})$ \\
\hline
\end{tabular}

observed periods is less than the number of parameters that define the stellar models (Giammichele et al. 2017a, b). This would be connected to the individual properties of the modes-illustrated in particular by their weight functions, which reflect the shape of the eigenfunctions of the modes - and the differential information contained in the distribution of these modes relative to each other. This is a very interesting aspect that deserves to be investigated in more detail.

With the aim of detecting a large number of pulsation periods, Nather et al. (1990) established the Whole Earth Telescope (WET). The studies by Winget et al. (1991) for PG1159-035 - the prototypical GW Vir star-and Winget et al. (1994) for GD 358 (V777 Her) — the brightest $\left(m_{V}=13.7\right)$ and best studied He-atmosphere WD pulsator-are excellent examples of the wealth of information that can be extracted from pulsating WDs from long, nearly uninterrupted data sets. As a more recent example of this, Bischoff-Kim et al. (2019) presented the results of three decades of observations of GD 358, which allowed the detection of 15 independent (13 consec- 
Table 4 ZZ Ceti stars (continuation of Table 2)

\begin{tabular}{|c|c|c|c|}
\hline Name & $T_{\text {eff }}(\mathrm{K})$ & $\log g$ & Magnitude \\
\hline GD 165 & 12220 & 8.11 & $14.3(\mathrm{~g})$ \\
\hline HE $1429-0343$ & 11290 & 8.00 & $16.0(\mathrm{~g})$ \\
\hline L 19-2 & 12070 & 8.13 & $13.4(\mathrm{GG})$ \\
\hline SDSS J144330.93+013405.8 & 10450 & 7.85 & $18.7(\mathrm{~g})$ \\
\hline SDSS J150207.02-000147.1 & 11090 & 7.75 & $18.7(\mathrm{~g})$ \\
\hline WD $1526+558$ & 10860 & 7.73 & $17.1(\mathrm{~g})$ \\
\hline HS $1531+7436$ & 13270 & 8.49 & $16.5(\mathrm{~g})$ \\
\hline SDSS J153332.96-020655.7 & 11390 & 8.04 & $16.4(\mathrm{~g})$ \\
\hline PG $1541+650$ & 11560 & 8.12 & $15.6(\mathrm{~g})$ \\
\hline SDSS J155438.35+241032.6 & 11470 & 8.49 & $17.5(\mathrm{~g})$ \\
\hline Ross 808 & 11120 & 7.98 & $14.4(\mathrm{~g})$ \\
\hline WD $1607+205$ & 11140 & 7.81 & $17.3(\mathrm{~g})$ \\
\hline SDSS J161218.08+083028.1 & 12250 & 8.29 & $17.8(\mathrm{~g})$ \\
\hline SDSS J161737.63+432443.8 & 11070 & 8.07 & $18.4(\mathrm{~g})$ \\
\hline SDSS J161837.25-002302.7 & 10292 & 7.97 & $19.3(\mathrm{~g})$ \\
\hline HS $1625+1231$ & 11690 & 8.06 & $16.1(\mathrm{~g})$ \\
\hline SDSS J164115.61+352140.6 & 12025 & 8.34 & $19.0(\mathrm{~g})$ \\
\hline G $226-29$ & 12510 & 8.35 & $12.2(\mathrm{~g})$ \\
\hline SDSS J165020.53+301021.2 & 10830 & 8.43 & $18.1(\mathrm{~g})$ \\
\hline GD 518 & 11760 & 8.97 & $17.3(\mathrm{~g})$ \\
\hline SDSS J170055.38+354951.1 & 11230 & 7.94 & $17.3(\mathrm{~g})$ \\
\hline SDSS J171113.01+654158.3 & 11130 & 8.47 & $16.9(\mathrm{~g})$ \\
\hline BPM 24754 & 10840 & 7.93 & $16.2(\mathrm{GG})$ \\
\hline SDSS J172428.42+583539.0 & 11640 & 7.88 & $17.6(\mathrm{~g})$ \\
\hline SDSS J173235.19+590533.4 & 10770 & 7.97 & $18.7(\mathrm{~g})$ \\
\hline HS $1824+6000$ & 11520 & 7.73 & $16.1(\mathrm{~g})$ \\
\hline G 207-9 & 12080 & 8.37 & $14.6(\mathrm{~g})$ \\
\hline KIC 7594781 & 11730 & 8.11 & $18.6(\mathrm{~g})$ \\
\hline KIC 10132702 & 11940 & 8.12 & $18.8(\mathrm{~g})$ \\
\hline KIC 4552982 & 10860 & 8.16 & $17.8(\mathrm{~g})$ \\
\hline KIC 4357037 & 10950 & 8.11 & $18.2(\mathrm{~g})$ \\
\hline KIC 8293193 & 12650 & 8.01 & $18.4(\mathrm{~g})$ \\
\hline KIC 11911480 & 12160 & 7.94 & $18.1(\mathrm{~g})$ \\
\hline KIC 4362927 & 11140 & 7.84 & $19.4(\mathrm{~g})$ \\
\hline G $185-32$ & 12470 & 8.10 & $13.0(\mathrm{~g})$ \\
\hline KIC 9162396 & 11070 & 8.06 & $18.5(\mathrm{~g})$ \\
\hline KIC 7766212 & 11890 & 8.01 & $16.8(\mathrm{~g})$ \\
\hline KIS J1945+4455 & 11590 & 8.04 & $17.2(\mathrm{~g})$ \\
\hline GD 385 & 11820 & 8.07 & $15.1(\mathrm{~g})$ \\
\hline GD 226 & 10730 & 8.06 & $16.4(\mathrm{~g})$ \\
\hline
\end{tabular}


Table 4 continued

\begin{tabular}{llll}
\hline Name & $T_{\text {eff }}(\mathrm{K})$ & $\log g$ & Magnitude \\
\hline SDSS J202857.52+771054.5 & 11940 & 8.38 & $19.0(\mathrm{~g})$ \\
WD 2102+233 & 11712 & 8.28 & $15.9(\mathrm{~g})$ \\
SDSS J212808.49-000750.8 & 11420 & 8.24 & $18.0(\mathrm{~g})$ \\
SDSS J213530.32-074330.7 & 10900 & 7.96 & $18.7(\mathrm{~g})$ \\
SDSS J214723.73-001358.4 & 12098 & 7.93 & $19.0(\mathrm{~g})$ \\
G 232-38 & 11590 & 8.02 & $16.8(\mathrm{~g})$ \\
WD 2148-291 & 11490 & 8.06 & $16.0(\mathrm{~g})$ \\
SDSS J215354.11-073121.9 & 11910 & 8.27 & $18.7(\mathrm{~g})$ \\
SDSS J215628.26-004617.2 & 10680 & 8.01 & $18.3(\mathrm{~g})$ \\
SDSS J215905.52+132255.7 & 11370 & 8.69 & $18.9(\mathrm{~g})$ \\
SDSS J220830.02+065448.7 & 11147 & 8.25 & $17.9(\mathrm{~g})$ \\
SDSS J220831.42+205909.66 & 11776 & 8.77 & $17.47(\mathrm{~g})$ \\
SDSS J220915.84-091942.5 & 11630 & 8.49 & $18.4(\mathrm{~g})$ \\
SDSS J221458.37-002511.7 & 11650 & 8.30 & $17.9(\mathrm{~g})$ \\
SDSS J223135.71+134652.8 & 11060 & 7.89 & $18.7(\mathrm{~g})$ \\
SDSS J223726.86-010110.9 & 11380 & 7.97 & $18.9(\mathrm{~g})$ \\
GD 244 & 11760 & 8.09 & $15.7(\mathrm{~g})$ \\
PG 2303+242 & 11500 & 8.07 & $15.3(\mathrm{~g})$ \\
SDSS J230726.66-084700.3 & 10970 & 8.21 & $18.9(\mathrm{~g})$ \\
SDSS J231934.52+515316.4 & 11435 & 8.45 & $19.37(\mathrm{~g})$ \\
G 29-38 & 11910 & 8.17 & $13.3(\mathrm{~g})$ \\
SDSS J233458.71+010303.1 & 11104 & 8.16 & $19.2(\mathrm{~g})$ \\
GD 1212 & 10970 & 8.03 & $13.3(\mathrm{~g})$ \\
G 30-20 & 11150 & 8.01 & $18.1(\mathrm{~g})$ \\
SDSS J235040.72-005430.9 & 10290 & 8.14 \\
EC 23487-2424 & 11560 & 8.09 & $15.3(\mathrm{~g})$ \\
\hline & & & \\
& & & \\
& & & \\
\hline
\end{tabular}

utive) $\ell=1$ pulsation modes. They also showed that the frequencies and amplitudes of these modes change with time.

The pulsation periods of WDs vary as a consequence of the evolution of these stars, giving place to a detectable rate of period change. The estimate of the rate of period change for pulsating WDs, a direct measurement of their evolutionary time scale (Winget et al. 1983), has progressed slowly because it demands huge observational time (Kepler et al. 2005; Costa and Kepler 2008; Sullivan and Chote 2015; Mukadam et al. 2013; Hermes et al. 2013c). Some recent applications of the rate of period change in ZZ Ceti stars to derive constraints on fundamental particles are presented in Sect. 3.9. On the other hand, the first ZZ Ceti in a detached WD with a main sequence star was discovered by Pyrzas et al. (2015). Cool ZZ Cetis, near the red edge of the instability strip, show long pulsation periods, and those observed -i.e., with significant amplitude - in general change with time. Bognár et al. (2018b) detected 14 
Table 5 ZZ Ceti stars (continuation of Table 2)

\begin{tabular}{|c|c|c|c|}
\hline Name & $T_{\text {eff }}(\mathrm{K})$ & $\log g$ & Magnitude \\
\hline LAMOST J004628.31+343319.9 & 11681 & 7.53 & $16.3(\mathrm{~g})$ \\
\hline LAMOST J062159.49+252335.9 & 11728 & 8.25 & $17.6(\mathrm{~g})$ \\
\hline LAMOST J010302.46+433756.2 & 11750 & 7.89 & $18.3(\mathrm{~g})$ \\
\hline LAMOST J013033.90+273757.9 & 14127 & 7.69 & $18.6(\mathrm{~g})$ \\
\hline KIC 4357037 & 12650 & 8.01 & $18.2(\mathrm{~g})$ \\
\hline KIC 4552982 & 10950 & 8.11 & $17.7(\mathrm{~g})$ \\
\hline KIC 7594781 & 11730 & 8.11 & $18.1(\mathrm{~g})$ \\
\hline KIC 10132702 & 11940 & 8.12 & $19.0(\mathrm{~g})$ \\
\hline KIC 11911480 & 11580 & 7.96 & $18.0(\mathrm{~g})$ \\
\hline KIC 60017836 & 10980 & 8.00 & $13.3(\mathrm{~g})$ \\
\hline EPIC 201355934 & 11770 & 7.97 & $17.8(\mathrm{~g})$ \\
\hline EPIC 201719578 & 11070 & 7.94 & $18.1(\mathrm{~g})$ \\
\hline EPIC 201730811 & 12480 & 7.96 & $17.1(\mathrm{~g})$ \\
\hline EPIC 201802933 & 12330 & 8.11 & $17.6(\mathrm{~g})$ \\
\hline EPIC 201806008 & 10910 & 8.02 & $14.9(\mathrm{~g})$ \\
\hline EPIC 206212611 & 10830 & 8.00 & $17.3(\mathrm{~g})$ \\
\hline EPIC 210397465 & 11200 & 7.71 & $17.6(\mathrm{~g})$ \\
\hline EPIC 211596649 & 11600 & 7.91 & $18.9(\mathrm{~g})$ \\
\hline EPIC 211629697 & 10600 & 7.77 & $18.3(\mathrm{~g})$ \\
\hline EPIC 211914185 & 13590 & 8.43 & $18.8(\mathrm{~g})$ \\
\hline EPIC 211916160 & 11510 & 7.96 & $18.9(\mathrm{~g})$ \\
\hline EPIC 211926430 & 11420 & 7.98 & $17.6(\mathrm{~g})$ \\
\hline EPIC 228682478 & 12070 & 8.18 & $18.2(\mathrm{~g})$ \\
\hline EPIC 229227292 & 11210 & 8.03 & $16.6(\mathrm{~g})$ \\
\hline EPIC 229228364 & 11030 & 8.03 & $17.8(\mathrm{~g})$ \\
\hline EPIC 220258806 & 12800 & 8.09 & $16.2(\mathrm{~g})$ \\
\hline EPIC 220347759 & 12770 & 8.08 & $17.6(\mathrm{~g})$ \\
\hline EPIC 220453225 & 11220 & 8.04 & 17.9 \\
\hline EPIC 229228478 & 12500 & 7.93 & $16.9(\mathrm{~g})$ \\
\hline SDSS J002945.75+14 4214.9 & 11310 & 7.95 & $18.20(\mathrm{GG})$ \\
\hline SDSS J002959.14+145814.2 & $10589 * *$ & 7.89 & $17.49(\mathrm{GG})$ \\
\hline WD J003116.51+474828.39 & $10444 * *$ & 8.04 & $18.36(\mathrm{GG})$ \\
\hline SDSS J004154.66-030802.5 & $10936^{* *}$ & 8.36 & $18.07(\mathrm{GG})$ \\
\hline SDSS J010025.55+421840.9 & $\cdots$ & $\cdots$ & $16.58(\mathrm{GG})$ \\
\hline SDSS J010528.74+020501.1 & $\cdots$ & $\cdots$ & $16.73(\mathrm{GG})$ \\
\hline SDSS J010539.14+321846.6 & $10737 * *$ & 8.08 & $18.07(\mathrm{GG})$ \\
\hline KUV 01595-1109 & 11062 & 8.14 & $16.89(\mathrm{GG})$ \\
\hline SDSS J022941.29-063842.7 & $9791 * *$ & 7.24 & $18.23(\mathrm{GG})$ \\
\hline
\end{tabular}


Table 5 continued

\begin{tabular}{|c|c|c|c|}
\hline Name & $T_{\text {eff }}(\mathrm{K})$ & $\log g$ & Magnitude \\
\hline WD J030648.49-172332.19 & $\cdots$ & $\cdots$ & $16.70(\mathrm{GG})$ \\
\hline WD J053212.77-432006.05 & $\cdots$ & $\cdots$ & $18.19(\mathrm{GG})$ \\
\hline SDSS J080609.19+111231.4 & $10987 * *$ & 7.90 & $18.11(\mathrm{GG})$ \\
\hline SDSS J084055.71+130329.4 & $\cdots$ & $\cdots$ & $17.22(\mathrm{GG})$ \\
\hline SDSS J084652.93+442638.6 & 11565 & 8.06 & $18.19(\mathrm{GG})$ \\
\hline SDSS J093250.56+554315.4 & $\cdots$ & $\cdots$ & $17.69(\mathrm{GG})$ \\
\hline SDSS J094851.43+512448.0 & 10252 & 7.80 & $18.57(\mathrm{GG})$ \\
\hline SDSS J103642.25+211527.9 & 11329 & 8.27 & $17.60(\mathrm{GG})$ \\
\hline CBS 130 & 11309 & 8.09 & $16.56(\mathrm{GG})$ \\
\hline SDSS J110505.94+583103.0 & $11256^{* * *}$ & 8.01 & $17.94(\mathrm{GG})$ \\
\hline WD J115057.43-055306.58 & $9611^{* *}$ & 7.38 & $17.44(\mathrm{GG})$ \\
\hline SDSS J120309.16+454520.3 & 11077 & 8.01 & $18.57(\mathrm{GG})$ \\
\hline SDSS J122155.79+050622.7 & $11720^{* *}$ & 7.00 & $17.90(\mathrm{GG})$ \\
\hline SDSS J124759.03+110703.0 & $\cdots$ & $\cdots$ & $19.35(\mathrm{GG})$ \\
\hline SDSS J124804.03+282103.8 & $10987 * *$ & 7.83 & $18.04(\mathrm{GG})$ \\
\hline WD $1452+600$ & $10962 * *$ & 7.91 & $17.18(\mathrm{GG})$ \\
\hline SDSS J150626.18+063845.9 & $\cdots$ & $\cdots$ & $16.60(\mathrm{GG})$ \\
\hline SDSS J150739.34+074828.5 & $10540 * *$ & 7.81 & $18.21(\mathrm{GG})$ \\
\hline SDSS J162724.67+392026.3 & $\cdots$ & $\cdots$ & $16.23(\mathrm{GG})$ \\
\hline SDSS J173351.49+341012.5 & $\cdots$ & $\cdots$ & $16.35(\mathrm{GG})$ \\
\hline WD J202838.13-060842.11 & $\cdots$ & $\cdots$ & $15.22(\mathrm{GG})$ \\
\hline WD J204127.11-041724.22 & $11114 * *$ & 8.34 & $18.31(\mathrm{GG})$ \\
\hline WD J212402.03-600100.05 & $\cdots$ & $\cdots$ & $17.97(\mathrm{GG})$ \\
\hline SDSS J215321.77+044020.0 & $10936 * *$ & 8.06 & $18.19(\mathrm{GG})$ \\
\hline SDSS J231536.88+192449.0 & $10540 * *$ & 7.85 & $17.99(\mathrm{GG})$ \\
\hline WD J231641.17-315352.74 & $11720 * *$ & 8.08 & $18.29(\mathrm{GG})$ \\
\hline SDSS J235010.36+201914.0 & $10786 * *$ & 7.86 & $17.41(\mathrm{GG})$ \\
\hline WD2246069 & 11512 & 7.99 & $16.94(\mathrm{GG})$ \\
\hline
\end{tabular}

**Photometric determination only, with large uncertainty

frequencies for HS 0733+4119, of which 8 are independent modes; for GD 154, 17 frequencies, with 4 independent modes, and for R808, the 28 frequencies discussed in Thompson et al. (2009) and 2 new ones.

For DBVs, the progress has been slower than for ZZ Cetis, first because the number of He atmosphere WDs is smaller, and also because the DBVs are hotter, and therefore evolve through the instability strip faster, and also due to that in the temperature estimate is much larger for DBs, in part caused by the $\mathrm{H}$ contamination in the atmosphere. With the consistent determination of the atmospheric parameters by Koester and Kepler (2015), the group led by Vanderbosch et al. (2018) increased the number of known DBV pulsators from 23 to 46, but their results are not yet published. Hermes et al. (2017b) discovered pulsations in the hot $\left(T_{\text {eff }} \sim 32000 \mathrm{~K}\right)$ DB PG 0112+104, 
Table 6 V777 Her stars and their effective temperatures, surface gravities, and magnitudes

\begin{tabular}{llll}
\hline Name & $T_{\text {eff }}(\mathrm{K})$ & $\log g$ & Magnitude \\
\hline KUV 05134+2605 & 24680 & 8.21 & $16.70(\mathrm{~g})$ \\
CBS 114 & 26050 & 7.98 & $17.50(\mathrm{~g})$ \\
PG 1115+158 & 23770 & 7.91 & $16.61(\mathrm{~g})$ \\
PG 1351+489 & 26010 & 7.91 & $16.52(\mathrm{~g})$ \\
PG 1456+103 & 24080 & 7.91 & $16.24(\mathrm{~g})$ \\
GD 358 & 24940 & 7.75 & $13.47(\mathrm{~g})$ \\
PG 1654+160 & 29410 & 7.97 & $16.40(\mathrm{~g})$ \\
PG 2246+121 & 27070 & 7.92 & $16.54(\mathrm{~g})$ \\
EC 20058-5234 & 25500 & 8.01 & $15.58(\mathrm{~g})$ \\
SDSS J034153.03-054905.8 & 25087 & 8.02 & $18.25(\mathrm{~g})$ \\
SDSS J085202.44+213036.5 & 25846 & 8.02 & $18.23(\mathrm{~g})$ \\
SDSS J094749.40+015501.8 & 23453 & 8.13 & $19.95(\mathrm{~g})$ \\
SDSS J104318.45+415412.5 & 26291 & 7.77 & $18.95(\mathrm{~g})$ \\
SDSS J122314.25+435009.1 & 23442 & 7.84 & $18.98(\mathrm{~g})$ \\
SDSS J125759.03-021313.3 & 25820 & 7.57 & $19.16(\mathrm{~g})$ \\
SDSS J130516.51+405640.8 & 24080 & 8.14 & $17.46(\mathrm{~g})$ \\
SDSS J130742.43+622956.8 & 23841 & 8.14 & $18.82(\mathrm{~g})$ \\
SDSS J140814.63+003838.9 & 26073 & 7.98 & $19.19(\mathrm{~g})$ \\
PG 0112+104 & 31300 & 7.8 & $15.21(\mathrm{~g})$ \\
EC 04207-4748 & 25970 & 7.79 & $15.25(\mathrm{GG})$ \\
EC 01585-1600 & 25500 & 7.88 & $14.40(\mathrm{~g})$ \\
WD J192904.6+444708 & 28480 & 7.89 & $18.38(\mathrm{~g})$ \\
EC 05221-4725 & 27900 & 7.78 & $16.72(\mathrm{GG})$ \\
SDSS J102106.69+082724.8 & 21629 & 7.96 & $18.23(\mathrm{GG})$ \\
WD J025121.71-125244.85 & $16567 * *$ & $18.01(\mathrm{GG})$ \\
SDSS J123654.96+170918.7 & $17673 * *$ & 7.51 & 7.98 \\
SDSS J132952.64+392150.5 & $20203^{* *}$ & & \\
\hline & & & 17 \\
\hline
\end{tabular}

The $T_{\text {eff }}$ and $\log g$ values have been derived using 3D model atmospheres (Cukanovaite et al. 2018). The letter in parentheses in the fourth column corresponds to the filter of the magnitude. Specifically, "g" is SDSS g magnitude and "GG" is Gaia G magnitude $* *$ Photometric determination only, with large uncertainty

expanding the DBV instability strip. In Table 6, we list the V777 Her stars known at the time of writing this review, along with their $T_{\text {eff }}, \log g$, and magnitudes. There are 4 new additional DBV stars discovered by Rowan et al. (2019) for which the effective temperatures and gravities have not been assessed yet.

The Kepler satellite observations, both main mission (Borucki et al. 2010) and K2 (two-wheel operation, Howell et al. 2014), increased the number of known WD pulsators by a factor of 2 (Hermes et al. 2017a), until it ran out of fuel by October 2018. It had $422200 \times 1024$ pixel charge coupled devices (CCD) to measure the stellar brightness variations. Due to data storage and downloading limitations, only 
512 stars were observed in short cadence mode - $58.85 \mathrm{~s}$ exposures — at each quarter, while the remaining observations were co-added on chip to $29.42 \mathrm{~min}$ long-cadence exposures (Gilliland et al. 2010). The extended time span of observations - at least 3 months for the main mission and around 80 days for each $\mathrm{K} 2$ cycle-allowed the determination of dozens of pulsation periods and the determination of rotation periods for different modes. In addition, the nearly continuous light curves eliminated spectral leakage in the Fourier Transforms (FTs), making period identification relatively easy. When launched, there were no known pulsating WDs in the Kepler field, and due to the telescope's relatively small $1.4 \mathrm{~m}$ primary mirror and fixed field of $10^{\circ} \times 10^{\circ}$, it took a large effort from the WD community to find pulsating WD candidates that could be observed in the limited number of targets in short cadence mode (Østensen et al. 2010, 2011a, b; Hermes et al. 2011; Greiss et al. 2016). An important aspect to be mentioned is the ability of Kepler to detect Nyquist-aliased pulsation signals, and the possibility to recover accurate pulsation periods to a precision similar to that of the K2 mode with the help of ground-based data (Bell et al. 2017c).

The long quasi-continuous observations of the Kepler satellite led to the unexpected discovery of outbursts in the WD pulsations by Bell et al. (2015), bringing another dimension to pulsation studies (Hermes et al. 2015; Bell et al. 2016; Hermes et al. 2017a; Bell et al. 2017b), probably caused by resonant mode coupling of the pulsations (Luan and Goldreich 2018, see Sect. 3.5). Still another benefit from the long quasicontinuous data was the possibility to estimate, for the first time, the natural width of the pulsation mode peaks (related to the stability) and shows that some modes are less stable than others. This had been hinted at from the WET observations of GD 358 (Kepler et al. 2003) and PG 1159-035 itself (Costa et al. 2008), but now shown in detail (Hermes et al. 2017b). One of the major observational results from the Kepler mission and its $\mathrm{K} 2$ extension is the discovery of a dichotomy of mode widths: lowfrequency modes with periods greater than roughly $800 \mathrm{~s}$ are generally incoherent over the length of observations, while higher-frequency modes are observed to be much more stable in phase and amplitude (Hermes et al. 2017a). Another example from space data is the K2 Campaign 10 on the GW Vir star PG 1159-035, with almost 50 days of coverage, showing at least 189 frequencies, and strong nonlinear effects throughout the observations. Finally, we mention the interesting finding of amplitude and frequency variations of the components of the triplets of frequencies caused by stellar rotation in the DBV star KIC 08626021 by Zong et al. (2016b). The observed modulations of the frequencies of this star are the clearest hints of nonlinear resonant couplings occurring in WDs stars identified so far. Resonant mode coupling signals have been also detected in the pulsating sdB star KIC 10139564 observed with the Kepler satellite (Zong et al. 2016b). These findings open a new window on the study of non-linear theory of stellar pulsations.

The successor of Kepler is the Transiting Exoplanet Survey Satellite (TESS, Ricker et al. 2015), composed of four $10.5 \mathrm{~cm}$ entrance pupil $24^{\circ} \times 24^{\circ}$ cameras, with $2 \mathrm{~min}$ cadence for guest observer mode, lasting at least 27 days. It will observe the 200000 brightest stars in $85 \%$ of the whole sky in 2 years. WD observations are limited because of their faintness. The main effort to get most of the brightest $(V<16)$ known WDs (and hot subdwarfs) observed within the TESS Asteroseismic Science Consortium 
Table 7 ELMV stars and their effective temperatures, surface gravities, magnitudes, and period ranges

\begin{tabular}{lllll}
\hline Name & $T_{\text {eff }}[\mathrm{K}]$ & $\log g[\mathrm{cgs}]$ & Magnitude & Period range [s] \\
\hline SDSS J184037.78+642312.3 & 9100 & 6.22 & $18.91(\mathrm{~g})$ & $2094-4890$ \\
SDSS J111215.82+111745.0 & 9590 & 6.36 & $16.35(\mathrm{~g})$ & $108-2855$ \\
SDSS J151826.68+065813.2 & 9900 & 6.80 & $17.54(\mathrm{~g})$ & $1335-3848$ \\
SDSS J161412.28+191219.4 $(*)$ & 8880 & 6.66 & $16.40(\mathrm{~g})$ & $1184-1263$ \\
SDSS J222859.93+362359.6(*) & 7870 & 6.03 & $16.83(\mathrm{~g})$ & $3254-6235$ \\
PSR J173853.96+033310.8 & 9130 & 6.55 & $21.3(\mathrm{~V})$ & $1788-4980$ \\
SDSS J161831.69+385415.2 & 9144 & 6.83 & $19.84(\mathrm{~g})$ & $5000-6100$ \\
SDSS J173521.69+213440.6(*) & 7940 & 5.76 & $16.12(\mathrm{~g})$ & $3363-4961$ \\
SDSS J213907.42+222708.9 $(*)$ & 7990 & 5.93 & $15.92(\mathrm{~g})$ & $2119-3303$ \\
SDSS J134336.44+082639.4 & 8100 & 5.97 & $16.27(\mathrm{~g})$ & 3600 \\
SDSS J222009.74-092709.9(*) & 8230 & 6.10 & $15.84(\mathrm{~g})$ & $2169-3591$ \\
\hline
\end{tabular}

The $T_{\text {eff }}$ and $\log g$ values have been derived using $3 \mathrm{D}$ model atmospheres. The letter in parentheses in the fourth column corresponds to the filter of the magnitude

(*) Probably not binary

(TASC) reserved 2 minute cadence slots has been coordinated by the TASC WG8 ${ }^{11}$. Independent efforts to find new pulsators among the bright targets in the WG8 lists have also been done. As an example of this, Bognár et al. (2018a) started looking for target WDs and there are a few WD pulsators down to $V=16.3$ already observed starting September 2018. There are no exclusive use data rights on TESS data, and the light curves are accessed through the Mikulski Archive for Space Telescopes (MAST), similar to the Kepler and $\mathrm{K} 2$ mission data.

The discovery of pulsations in ELMs (Hermes et al. 2012) and pre-ELMs (Maxted et al. 2014b) (see Sect. 3.6), and even Blue Large-Amplitude Pulsators (BLAPs, Pietrukowicz et al. 2017, see Sect. 3.7), is another chapter on the asteroseismological study of the by-products of binary interactions. DA WD stars with masses $M_{\star} \leq$ $0.45 M_{\odot}$ and $T_{\text {eff }}<20000 \mathrm{~K}$ are Low-Mass (LM) WDs, and if $M_{\star} \lesssim 0.18-0.20 M_{\odot}$ they are called Extremely Low-Mass (ELM) WDs. They have been found by Kilic et al. (2011), Brown et al. (2010, 2012, 2013, 2016), and Gianninas et al. (2014, 2015). Hermes et al. (2012, 2013b, d), Kilic et al. (2015, 2018), Bell et al. (2017a, 2018), Pelisoli et al. (2018b, 2019) found pulsations in eleven of these ELMs, similar to the $g$-mode pulsations seen in DAVs (Van Grootel et al. 2013), although with much longer periods. Maxted et al. (2014a) found 17 pre-ELMs, i.e., He-core WD precursors, and Maxted et al. $(2013,2014 \mathrm{~b})$ and Gianninas et al. (2016) report pulsations in five of them. In Tables 7 and 8, we show the list of ELMVs and pre-ELMVs, respectively, detected at the time of writing this review.

The prototype of the DQV class, SDSS J142625.71+575218.3, was discovered by Montgomery et al. (2008). Barlow et al. (2008) and Dunlap et al. (2010) found three others, SDSS J220029.08-074121.5, SDSS J234843.30-094245.3, and SDSS J133710.19-002643.6. Dufour et al. (2011) discovered the fifth DQV star,

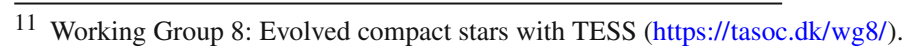


Table 8 Pre-ELMV stars and their effective temperatures, surface gravities, magnitudes, and period ranges

\begin{tabular}{lllll}
\hline Star & $T_{\text {eff }}[\mathrm{K}]$ & $\log g[\mathrm{cgs}]$ & Magnitude $[\mathrm{s}]$ & Period range \\
\hline SDSS J115734.46+054645.6 & 11870 & 4.81 & $19.9(\mathrm{~g})$ & 364 \\
SDSS J075610.71+670424.7 & 11640 & 4.90 & $16.3(\mathrm{~g})$ & $521-587$ \\
WASP J024743.37-251549.2 & 11380 & 4.576 & $12.1(\mathrm{~g})$ & $380-420$ \\
SDSS J114155.56+385003.0 & 11290 & 4.94 & $19.1(\mathrm{~g})$ & $325-368$ \\
KIC 9164561 $(*)$ & 10650 & 4.86 & $13.7(\mathrm{~g})$ & $3018-4668$ \\
WASP J162842.31+101416.7 & 9200 & 4.49 & $13.0(\mathrm{~g})$ & $668-755$ \\
SDSS J173001.94+070600.25(*) & 7972 & 4.25 & $16.4(\mathrm{~g})$ & 3367 \\
SDSS J145847.02+070754.46(*) & 7925 & 4.25 & $15.2(\mathrm{~g})$ & $1634-3279$ \\
SDSS J131011.61-014233.0 $(*)$ & 8224 & 5.33 & $16.6(\mathrm{~g})$ & $2100-3100$ \\
SDSS J075738.94+144827.50(*) & 8180 & 4.75 & $15.0(\mathrm{~g})$ & $803-2982$
\end{tabular}

The letter in parentheses in the fourth column corresponds to the filter of the magnitude

(*) Unconfirmed as a pre-ELM WD (see text)

SDSS J115305.55+005646.2. Williams et al. (2013) discuss the variability in the strongly magnetic DQ WD SDSS J103655.39+652252.2, but it is not evident if these are bona fide pulsations or more probably caused by cool spots in rotating magnetic stars (Williams et al. 2016; Dupuis 2018). At present, 6 stars of this category exist (see Table 9).

WDs in cataclysmic variables (CVs) also show pulsations, as discovered by Warner and van Zyl (1998) in the quiescent phase of the dwarf nova GW Librae. Since then, pulsations have been detected in nearly twenty dwarf novae (see Szkody et al. 2013, 2015; Toloza et al. 2016), that define the GW Lib class of pulsating WDs. The prototype star, GW Librae, showed nonradial pulsations during quiescent phases before and after the 2007 nine magnitude outburst. The location of the instability strip of the class of GW Lib stars is affected by accretion, which changes the temperature structure of the envelope and therefore the driving region. Theoretical models indicate excitation of $g$ modes due to the $\mathrm{H} / \mathrm{HeI}$ ionization zone, but enhanced $\mathrm{He}$ abundance due to accretion also can drive pulsations as a result of the subsurface HeII partial ionization zone (Arras et al. 2006; Van Grootel et al. 2015). On the other hand, Saio (2019) proposes that toroidal $r$ modes are also detected - analyzing the substructure of the pulsation peaks-and concludes the outburst in the star GW Librae increased the rotation rate of the $\mathrm{H}$ envelope significantly.

Gaia Data Release 2 (DR2; Gaia Collaboration et al. 2018a,b) is a new source of detailed information for distances, and therefore radii and masses, for the known WDs. It also allowed the discovery of a few hundred thousand probable WDs (Gentile Fusillo et al. 2019; Kepler et al. 2019), and consequently of WD pulsator candidates, as pulsation appears to be a natural phenomenon during WD cooling. Once Gaia make spectrophotometry and light curves available, it will be possible to confirm WD pulsators directly without the use of dedicated follow-ups, although it depends on the number of measurements and time scale (frequency) of the Gaia measurements. Fig. 3 shows the color-magnitude diagram for the stars detected with parallax/error $>10 \sigma$ in Gaia DR2, and the location of the WD variables. Recently, using observations with 


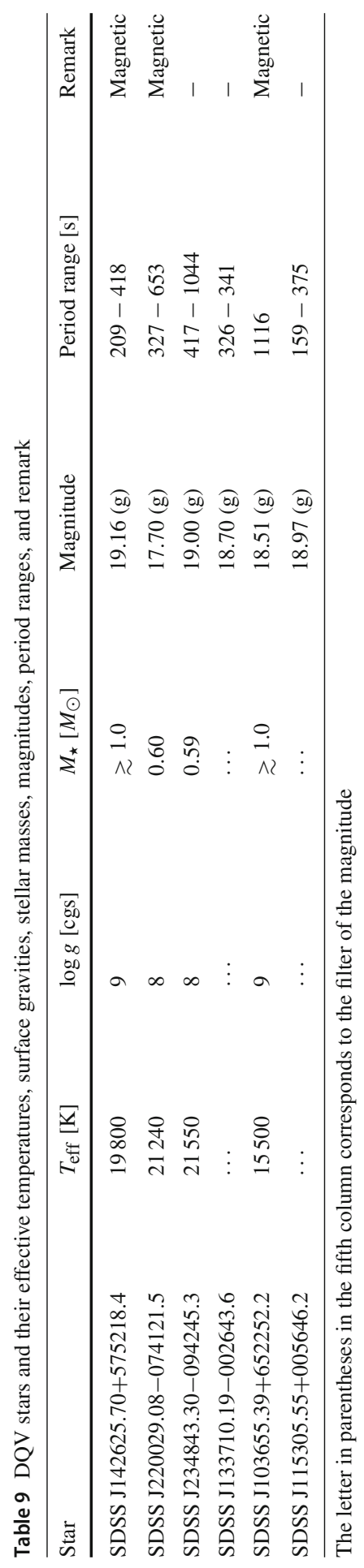




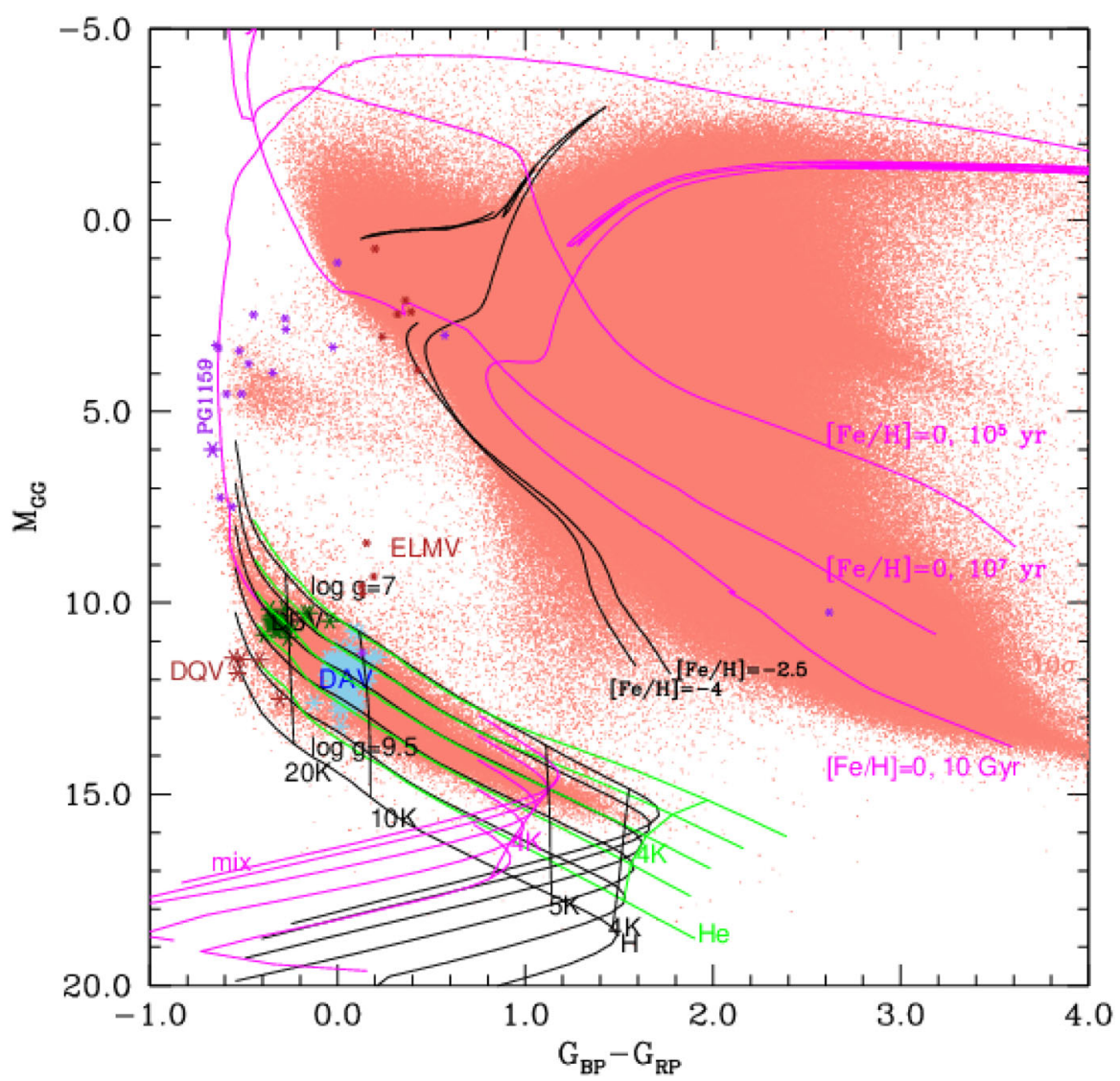

Fig. 3 Color-magnitude diagram for the known variables DAVs, DBVs, pulsating PG1159 stars (including PNNVs), ELMVs, and variable DQVs, from Gaia DR2 distances and colors. The background is composed of the stars in Gaia DR2 with parallax/error > 10. The lines are MIST (MESA Isochrones \& Stellar Tracks; Choi et al. 2018) isochrones with solar metallicity, $[\mathrm{Fe} / \mathrm{H}]=0$, and also $[\mathrm{Fe} / \mathrm{H}]=-2.5$, and $[\mathrm{Fe} / \mathrm{H}]=-4$, where $[\mathrm{Fe} / \mathrm{H}]=\left[\log (\mathrm{Fe} / \mathrm{H})_{\star}-\log (\mathrm{Fe} / \mathrm{H})_{\odot}\right]$. The adopted age is for the low-metallicity isochrones. Three different ages are considered in the solar case. In the WD region, the lines are the pure $\mathrm{H}$, pure $\mathrm{He}$, and mixed models from Carrasco et al. (2014). The Gaia DR2 data are less reliable for stars fainter than $M_{\mathrm{G}}>19$. This is because both the photometry and the parallax available on Gaia DR2 are less reliable because of background noise in the Gaia data

the Gaia satellite, Tremblay et al. (2019) have confirmed that the cores of cooling WDs undergo crystallization, as predicted half a century ago ( van Horn 1968).

In the case of pulsating PG 1159 stars, also called GW Vir stars, Table 3 in Fontaine and Brassard (2008) (see also Table 5 of Althaus et al. 2010b), requires only minor revisions. We have to add two objects to the list. On one hand, the GW Vir star SDSS J075415.12+085232.18, discovered by Kepler et al. (2014) and characterized by $T_{\text {eff }}=120000 \pm 10000 \mathrm{~K}, \log g=7.0 \pm 0.3, M_{\star}=0.52 \pm 0.02 M_{\odot}$, and a dominant period of $\sim 525 \mathrm{~s}$. On the other hand, the GW Vir star SDSS J0349-0059, discovered to be pulsating by Woudt et al. (2012), and characterized by $T_{\text {eff }}=90000 \pm 900 \mathrm{~K}$, 
$\log g=7.5 \pm 0.01, M_{\star}=0.543 \pm 0.004 M_{\odot}$, and periods in the range [301 - -964] s. This star was analyzed asteroseismologically by Calcaferro et al. (2016). Finally, we have to subtract VV47-a supposed PNNV star reported to be variable by González Pérez et al. (2006) - from the list of GW Vir stars. In fact, a re-analysis by Sowicka et al. (2018) revealed that this star does not show variability according to the new photometric data, but neither with the original data of its discovery!

Another issue that has experienced a remarkable development is the derivation of the rotation rates of WDs through asteroseismology. Slow, solid-body rotation of a star produces a set of equally spaced frequencies, with a separation between each component of the multiplet given by $\delta \sigma_{k, \ell, m}=m\left(1-C_{k \ell}\right) \Omega_{\mathrm{R}}$. This rotational splitting of frequencies is found in a large number of pulsating WDs. This allows to estimate the rotation period of the $\operatorname{star}\left(P_{\mathrm{R}}=2 \pi / \Omega_{\mathrm{R}}\right)$ as well as to identify the harmonic degree $\ell$ and the azimuthal order $m$ of the modes (see Althaus et al. 2010b, for details). In general, the measured rotation period for single WDs ranges from 1 hour to 18 days, with a median around 1 day (Kawaler 2015). We show in Table 10 a compilation of pulsating WDs and pre-WDs with rotation periods derived from asteroseismology. The fastest single WD rotator from asteroseismological measurements (Table 10) is the $0.79 M_{\odot}$ DAV SDSS J161218.08+083028.1 discovered by Castanheira et al. (2013), assuming that the two observed periods at $115.0 \mathrm{~s}$ and $117.0 \mathrm{~s}$ are two components of a rotation triplet. This star seems to be rotating with a period of $0.93 \mathrm{~h}$ ! Differential rotation in WDs was studied by Winget et al. (1991, 1994), Kawaler et al. (1999), Charpinet et al. (2009), Córsico et al. (2011), Fontaine et al. (2013) and Hermes et al. (2017b), using the change in rotation splitting of non-radial pulsations. In particular, Kawaler et al. (1999) (see, also, Córsico et al. 2011) employed asteroseismological inversion methods for the inversion of the rotation profiles of GW Vir and V777 Her stars. Hermes et al. (2017b) have been able to assess, for the first time in a systematic way, WD rotation as a function of the stellar mass. In particular, they found that WDs with masses between 0.51 and $0.74 M_{\odot}$ have a mean rotation period of $35 \mathrm{hr}$. The assessment of the rotation periods of WDs through asteroseismology provides final boundary conditions to the internal angular-moment evolution of isolated stars. The longest rotation period determined is for the hot polluted ZZ Ceti (DAV) GD 133 by $\mathrm{Fu}$ et al. (2019). In particular, the WD rotation rates as a function of mass can shed light on the unknown angular-momentum transport mechanism coupling red giant cores to their envelopes (Deheuvels et al. 2012; Tayar and Pinsonneault 2013; Mosser et al. 2014; Cantiello et al. 2014; den Hartogh et al. 2019). The longest rotation period determined is for the hot polluted (DAZ) ZZ Ceti (DAV) GD 133 by Fu et al. (2019). The metals imply ongoing accretion of planetesimals.

\subsection{Asteroseismic approaches}

Up to now, two main standpoints have been adopted to perform forward asteroseismic modeling of pulsating WD stars. One approach considers WD models characterized by parameterized chemical composition profiles, while the other technique involves fully evolutionary models constructed with chemical profiles resulting from all the processes experienced during the evolution of the WD progenitors. The former approach consti- 
Table 10 Rotation periods of WDs as determined via asteroseismology

\begin{tabular}{|c|c|c|c|c|}
\hline Star & $P_{\text {rot }}[\mathrm{h}]$ & $T_{\text {eff }}[\mathrm{K}]$ & Type & $M_{\star}\left[M_{\odot}\right]$ \\
\hline RX J2117.1+3412 & 28 & 170000 & GW Vir & 0.72 \\
\hline PG 1159-035 & 33 & 140000 & GW Vir & 0.54 \\
\hline NGC 1501 & 28 & 134000 & [WCE] & 0.56 \\
\hline PG $2131+066$ & 5 & 95000 & GW Vir & 0.55 \\
\hline PG $1707+427$ & 16 & 85000 & GW Vir & 0.53 \\
\hline PG $0122+200$ & 37 & 80000 & GW Vir & 0.53 \\
\hline SDSS J0349-0059 & 9.8 & 90000 & GW Vir & 0.54 \\
\hline PG 0112+104 & 10.17 & 31040 & DBV & 0.58 \\
\hline KIC 8626021 & 43 & 29,700 & DBV & 0.56 \\
\hline EC $20058-5234$ & 2 & 25500 & DBV & 0.65 \\
\hline GD 358 & 29 & 23,740 & DBV & 0.54 \\
\hline SDSS J083702.16+185613.4 & 1.13 & 13590 & ZZ Ceti & 0.88 \\
\hline G 226-29 & 9 & 12510 & ZZ Ceti & 0.83 \\
\hline G $185-32$ & 15 & 12470 & ZZ Ceti & 0.67 \\
\hline GD 133 & 169 & $12400(*)$ & ZZ Ceti & 0.63 \\
\hline SDSS J113655.17+040952.6 & 2.6 & 12330 & ZZ Ceti & 0.55 \\
\hline SDSS J161218.08+083028.1 & 0.93 & 12330 & ZZ Ceti & 0.79 \\
\hline Ross 548 & 37 & 12300 & ZZ Ceti & 0.63 \\
\hline GD 165 & 50 & 12220 & ZZ Ceti & 0.68 \\
\hline LP $133-144$ & 41.8 & 12,150 & ZZ Ceti & 0.59 \\
\hline KIC 11911480 & 86.4 & 12160 & ZZ Ceti & 0.58 \\
\hline L $19-2$ & 13 & 12070 & ZZ Ceti & 0.69 \\
\hline HS $0507+0435$ & 38 & 12010 & ZZ Ceti & 0.73 \\
\hline EC 14012-1446 & 14.4 & 12020 & ZZ Ceti & 0.72 \\
\hline KUV $11370+4222$ & 5.56 & 11940 & ZZ Ceti & 0.72 \\
\hline G 29-38 & 32 & 11910 & ZZ Ceti & 0.72 \\
\hline EPIC 220274129 & 12.7 & 11810 & ZZ Ceti & 0.62 \\
\hline KUV 02464+3239 & 90.7 & 11620 & ZZ Ceti & 0.70 \\
\hline HL Tau 76 & 53 & 11470 & ZZ Ceti & 0.55 \\
\hline SDSS J171113.01+654158.3 & 16.4 & 11130 & ZZ Ceti & 0.90 \\
\hline GD 154 & 50.4 & 11120 & ZZ Ceti & 0.65 \\
\hline KIC 4552982 & 15.0 & 10860 & ZZ Ceti & 0.71 \\
\hline SDSS J094000.27+005207.1 & 11.8 & 10,590 & ZZ Ceti & 0.82 \\
\hline KIC 435703719 & 22.0 & 12650 & ZZ Ceti & 0.62 \\
\hline KIC 455298219 & 18.4 & 10950 & ZZ Ceti & 0.67 \\
\hline KIC 759478119 & 26.8 & 11730 & ZZ Ceti & 0.67 \\
\hline KIC 1013270219 & 11.2 & 11940 & ZZ Ceti & 0.68 \\
\hline KIC 1191148019 & 74.7 & 11580 & ZZ Ceti & 0.58 \\
\hline EPIC 6001783623 & 6.9 & 10980 & ZZ Ceti & 0.57 \\
\hline EPIC 20171957811 & 26.8 & 11070 & ZZ Ceti & 0.57 \\
\hline
\end{tabular}


Table 10 continued

\begin{tabular}{lllll}
\hline Star & $P_{\text {rot }}[\mathrm{h}]$ & $T_{\text {eff }}[\mathrm{K}]$ & Type & $M_{\star}\left[M_{\odot}\right]$ \\
\hline EPIC 20173081111 & 2.6 & 12480 & ZZ Ceti & 0.58 \\
EPIC 20180293311 & 31.3 & 12330 & ZZ Ceti & 0.68 \\
EPIC 20180600811 & 31.3 & 10910 & ZZ Ceti & 0.61 \\
EPIC 21039746503 & 49.1 & 11200 & ZZ Ceti & 0.45 \\
EPIC 21159664908 & 81.8 & 11600 & ZZ Ceti & 0.56 \\
EPIC 21162969708 & 64.0 & 10600 & ZZ Ceti & 0.48 \\
EPIC 21191418508 & 1.1 & 13590 & ZZ Ceti & 0.88 \\
EPIC 21192643009 & 25.4 & 11420 & ZZ Ceti & 0.59 \\
EPIC 22868247808 & 109.1 & 12070 & ZZ Ceti & 0.72 \\
EPIC 22922729213 & 29.4 & 11210 & ZZ Ceti & 0.62 \\
EPIC 22020462601 & 24.3 & 11620 & ZZ Ceti & 0.71 \\
EPIC 22025880601 & 30.0 & 12800 & ZZ Ceti & 0.66 \\
EPIC 22034775900 & 31.7 & 12770 & ZZ Ceti & 0.66 \\
EPIC 220274129 & 12.7 & 11810 & ZZ Ceti & 0.62 \\
\hline
\end{tabular}

The $T_{\text {eff }}$ values have been converted to $3 \mathrm{D}$ model atmospheres

(*) Asteroseismological value of $T_{\text {eff }}$ and $\log g$ because of the observed metal polution

tutes a powerful forward method with the flexibility of allowing a full exploration of the parameter space (the total mass, the mass of the $\mathrm{H}$ and He envelopes, the thickness of the chemical transition regions, the core chemical structure and composition, etc) to find an optimum asteroseismological solution (see Bradley 1998, 2001; BischoffKim et al. 2008a, 2014, 2019; Fu et al. 2013; Bognár et al. 2016; Giammichele et al. 2017a, b, among others). In particular, Giammichele et al. (2017a,b) present a new prescription for parameterizing the chemical profiles in the core of WDs which is based on Akima splines. The method, in principle, allows for period-to-period fits (theoretical periods minus observed periods) with differences smaller than the uncertainties in the observed periods. This technique has been applied by Giammichele et al. (2018) to the DBV star KIC 08626021 monitored extensively by the Kepler spacecraft. We describe this specific application in some detail here due to the relevance of this asteroseismological technique. Giammichele et al. (2018) have derived the chemical stratification of ${ }^{16} \mathrm{O},{ }^{12} \mathrm{C}$ and ${ }^{4} \mathrm{He}$ in KIC08626021 using archival data. They find an asteroseismological model for this star whose ${ }^{16} \mathrm{O}$ content and the extent of its core exceed the predictions of current models of DB WDs derived from fully evolutionary computations. The chemical profiles of the asteroseismological model are displayed in Fig. 4. This model is characterized by $T_{\text {eff }}=29968 \pm 200 \mathrm{~K}$ and $\log g=7.917 \pm 0.009$, which closely match the independent measurements obtained from spectroscopy $\left(T_{\text {eff }}=29360 \pm 780 \mathrm{~K}\right.$ and $\left.\log g=7.89 \pm 0.05\right)$. The central homogeneous part of the core of the asteroseismological model has a mass of $0.45 M_{\odot}$, and is composed of $\sim 86 \%{ }^{16} \mathrm{O}$ by mass. These values are, respectively, $40 \%$ and $15 \%$ larger than those predicted for typical DB WD models. The total ${ }^{16} \mathrm{O}$ content of the 


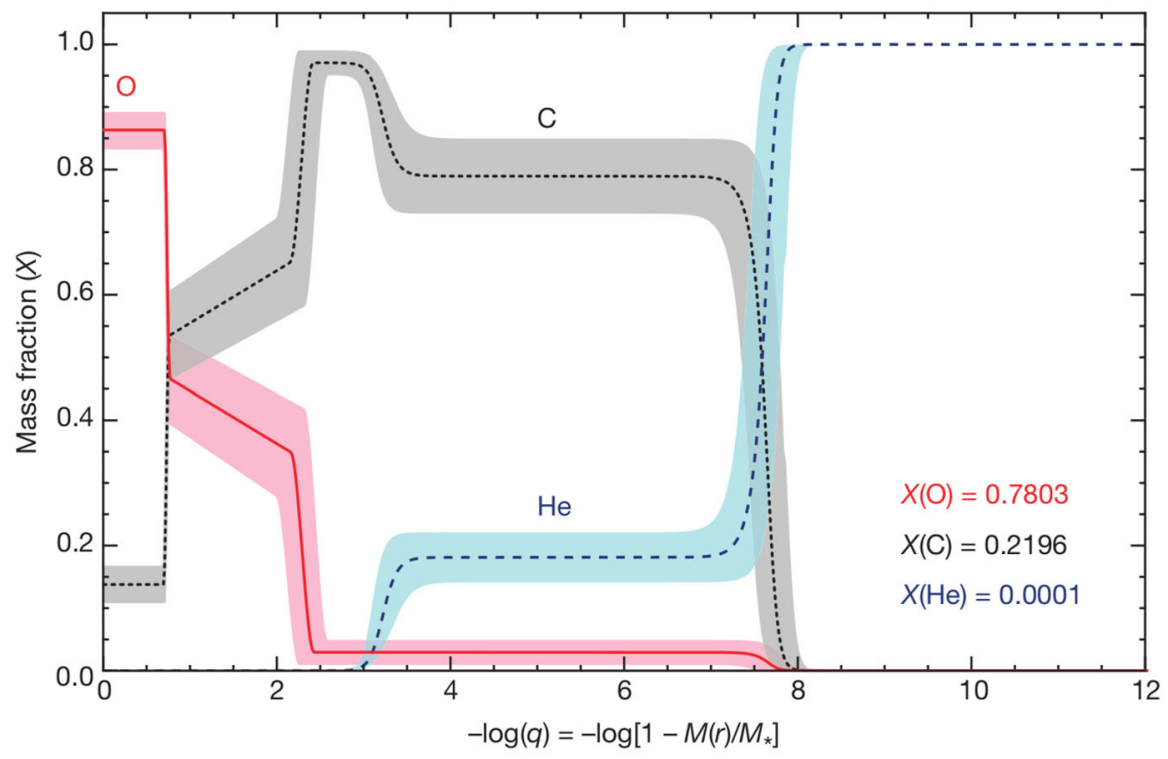

Fig. 4 Abundance by mass of ${ }^{16} \mathrm{O}$ (in red), ${ }^{12} \mathrm{C}$ (in gray) and ${ }^{4} \mathrm{He}$ (in blue) in terms of the outer mass fraction, corresponding to the asteroseismological model of KIC08626021. The shaded areas around each curve are the estimated $1 \sigma$ errors. The total (integrated) mass fractions for each chemical species are indicated at the bottom right of the plot. Image reproduced with permission from Giammichele et al. (2018), copyright by Macmillan

WD core reaches $78.0 \pm 4.2 \%$, much higher than the expected value of around $64 \%$ for a standard evolutionary DB WD model of the same mass.

A disturbing feature of the method described above is that it can lead to asteroseismological models characterized by chemical structures that cannot be reconciled with the predictions of the currently accepted scenarios of WD formation. For instance, the derived asteroseismological models may have a pure (or nearly pure) ${ }^{12} \mathrm{C}$ bufferwhich is difficult to predict from the existing channels of WD formation; see De Gerónimo et al. (2019b), submitted - or central abundances of ${ }^{12} \mathrm{C}$ and ${ }^{16} \mathrm{O}$ that are at variance with the current uncertainty of the ${ }^{12} \mathrm{C}(\alpha, \gamma){ }^{16} \mathrm{O}$ reaction rate (see Fig. 4). In addition, as precise as they have become, parameterized approaches rely on an educated guess of the internal composition profiles, due to the large number of parameters involved and the small number of periods typically available.

The second asteroseismological approach for WDs was developed at La Plata Observatory and is based on non-static WD evolutionary models that result from the complete evolution of the progenitor stars. This method has been already applied to GW Virginis stars (see, e.g., Córsico et al. 2007a, b, 2008, 2009a; Calcaferro et al. 2016, and references therein), to DBV WDs (see, e.g., Córsico et al. 2012a, 2014; Bognár et al. 2014), and recently to ELMV stars (Calcaferro et al. 2017b, 2018b). Regarding ZZ Ceti stars, this approach has been employed by Romero et al. (2012, $2013,2017)$. In the context of this approach, the chemical structure of WD models is consistent with the pre-WD evolution. This is a crucial aspect because WD models with 
consistent and detailed chemical profiles from the center to the surface are needed to correctly assess the adiabatic pulsation periods and also the mode-trapping properties of ZZ Ceti stars. These chemical profiles are computed from the complete evolution of the progenitor stars from the ZAMS, through the thermally pulsing and mass-loss phases on the AGB, and from time-dependent element diffusion predictions during the WD stage. For realistic asteroseismic inferences, this method requires a quantification of the uncertainties inherent to WD progenitor evolution and an assessment of their impact on the pulsational expectations.

A notable example of application of this second approach to DAV stars is the asteroseismological study of $44 \mathrm{ZZ}$ Ceti stars extracted from a sample of bright stars-for which the surface parameters are accurately known-performed by Romero et al. (2012). These authors employed a large grid of fully evolutionary models characterized by consistent chemical profiles covering a wide range of stellar masses, thicknesses of the $\mathrm{H}$ envelope and effective temperatures. The different thicknesses of the $\mathrm{H}$ envelope were artificially obtained from the canonical (thickest) envelopes. One of the main results of Romero et al. (2012) is a strong suggestion of the existence of a range of thicknesses of the $\mathrm{H}$ envelope in the studied $\mathrm{ZZ}$ Ceti stars, with a distribution characterized by a strong peak at thick envelopes $\left[\log \left(M_{\mathrm{H}} / M_{\star}\right) \sim-4.5\right]$ and another much less pronounced peak at very thin envelopes $\left[\log \left(M_{\mathrm{H}} / M_{\star}\right) \sim-9.5\right]$, with an evident paucity for intermediate thicknesses; see upper panel of Fig. 5. The expected range of $\mathrm{H}$-envelope thickness, that is in agreement with the seismological results of Castanheira and Kepler (2009), is extremely relevant and is in line with the spectroscopic finding of Tremblay and Bergeron (2008), that about $15 \%$ of the DA WDs with $10000 \mathrm{~K} \lesssim T_{\text {eff }} \lesssim 15000 \mathrm{~K}$ should have $\mathrm{H}$ envelopes in the range $-10 \leq \log \left(M_{\mathrm{H}} / M_{\star}\right) \leq-8$. These two pieces of evidence reinforce the idea that a non-negligible fraction of DA WDs could harbor thin H envelopes. Because the standard theory of stellar evolution predicts the formation of WDs with thick H envelopes, then the following question arises: which are the possible channels of evolution that can lead to the formation of DA WDs with a range of envelopes of $\mathrm{H}$ ? In the case of single progenitors, the expected theoretical predictions are displayed in Fig. 5. In the lower panel of this figure, the stellar mass in terms of the H envelope thickness corresponding to the asteroseismological models derived by Romero et al. (2012) is shown. The thick brown line corresponds to the thickest $\mathrm{H}$ envelopes possible (canonical envelopes) corresponding to progenitor stars with nearly solar metallicity, $Z=0.01$. The thick green line, in turn, corresponds to the canonical H envelopes of DA WD models coming from progenitor stars with sub-solar metallicity $(Z=0.001)$. There is a widespread belief that progenitor metallicity and/or enhanced winds on the AGB and H-burning post-AGB phases could be at the root of accounting for the existence of WDs with very thin $\mathrm{H}$ envelopes (see Althaus et al. 2015, for a thorough discussion). From inspection of Fig. 5, it becomes clear that, according to fully evolutionary computations, the metallicity of the WD progenitors cannot be the explanation for the formation of WDs with $\mathrm{H}$ envelopes thinner than the canonical ones; see Miller Bertolami et al. (2013), Althaus et al. (2015) and Miller Bertolami et al. (2017). These authors also show that neither the action of enhanced winds during the AGB and post-AGB phases can be responsible for the thin $H$ envelopes in DA WDs. Rather, the explanation for the existence of WDs with thinner $\mathrm{H}$ envelopes than the canonical 

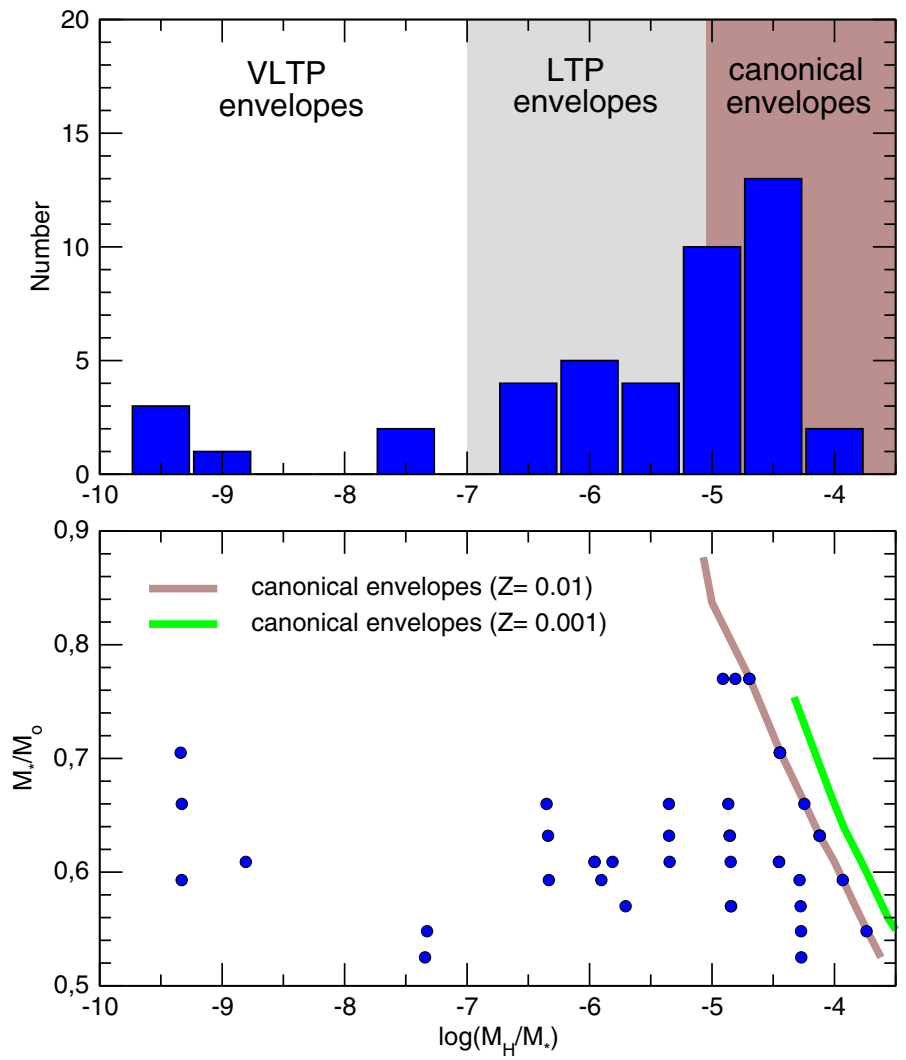

Fig. 5 Upper panel: histogram of the H envelope thickness distribution corresponding to the asteroseismological models of the sample of 44 ZZ Ceti stars analyzed in Romero et al. (2012). The regions marked with different colors (brown, gray and white) refer to three possible evolutionary origins (single progenitors) of the WDs according to the value of the thickness of their H envelopes. The acronyms LTP and VLTP stand for Late Thermal Pulse and Very Late Thermal Pulse, respectively (see Sect. 2.1). Lower panel: stellar mass in terms of the $\mathrm{H}$ envelope mass for the same sample of ZZ Ceti stars. The thick brown (green) line corresponds to the canonical envelopes for post-AGB WDs at the beginning of the cooling track coming from progenitor stars with $Z=0.01(Z=0.001)$ at the ZAMS

ones can be found in connection with the occurrence of late thermal pulses experienced by progenitors after departing from the TP-AGB phase; specifically LTP and VLTP events, as proposed by Althaus et al. (2005) and Miller Bertolami et al. (2017) (see also Sect. 2 for details). The range of $\mathrm{H}$ envelope mass expected from these scenarios is schematically illustrated in the upper panel of Fig. 5.

The sample of ZZ Ceti stars studied in Romero et al. (2012) includes the archetypal ZZ Ceti star G117-B15A. For this star, a unique asteroseismological solution, characterized by $T_{\text {eff }}=11,985 \pm 200 \mathrm{~K}, \log g=8.00 \pm 0.09$ and $M_{\star}=$ $0.593 \pm 0.007 M_{\odot}$-in excellent agreement with the spectroscopic determinationswas found. For the first time, the degeneracy of the asteroseismological solutions for this star reported by previous studies (e.g., Bradley 1998) regarding the thickness of the $\mathrm{H}$ envelope was broken. The best-fit model has a $\mathrm{H}$ envelope with 

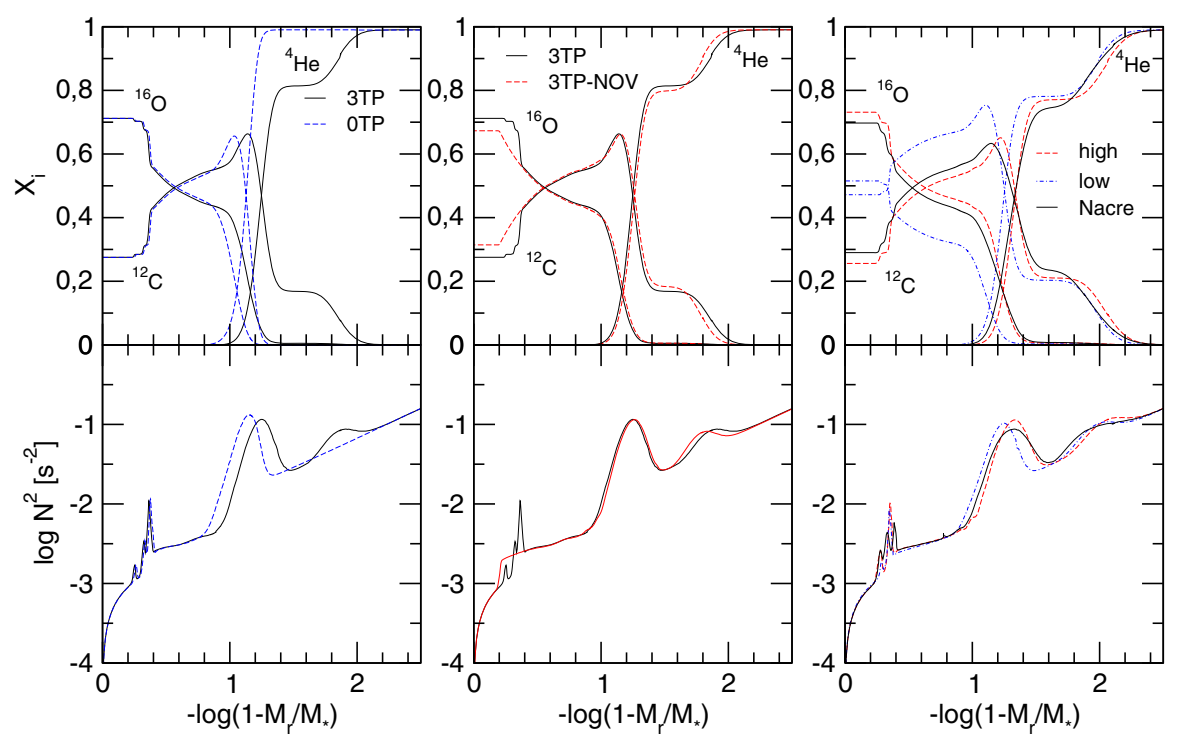

Fig. 6 Left panels: inner ${ }^{16} \mathrm{O},{ }^{12} \mathrm{C}$ and ${ }^{4} \mathrm{He}$ abundance distribution (upper panel) and the logarithm of the squared Brunt-Väisälä frequency (lower panel) in terms of the outer mass fraction for two DA WD models at $T_{\text {eff }} \sim 12000 \mathrm{~K}$. 0TP and 3TP refer to the expected chemical profiles when the progenitor departed from the AGB before the occurrence of the first TP and at the end of the third TP, blue dashed and black solid lines, respectively. Middle panels: same as left panels, but for models at the third TP calculated with OV (3TP; black solid line) and without OV (3TP-NOV; red dashed line) during core He burning. Right panels: same as left panels, but for models computed with three different ${ }^{12} \mathrm{C}(\alpha, \gamma){ }^{16} \mathrm{O}$ reaction rates: Angulo et al. (1999) (Nacre; black solid line), the higher (high; red dashed line), and the lower (low; blue dot-dashed line) reaction rates from Kunz et al. (2002)

$M_{\mathrm{H}}=(1.25 \pm 0.7) \times 10^{-6} M_{\star}$, about two orders of magnitude thinner than the value predicted by canonical evolutionary computations, of $M_{\mathrm{H}} \sim 10^{-4} M_{\star}$ at this stellar-mass value.

As stated before, reliable asteroseismological inferences of WD parameters require an assessment of the uncertainties of the predictions of WD progenitor evolution. In this sense, De Gerónimo et al. (2017, 2018) have explored for the first time the impact of the occurrence of TPs on WD progenitors, the uncertainty in the ${ }^{12} \mathrm{C}(\alpha, \gamma){ }^{16} \mathrm{O}$ cross section, and the occurrence of extra mixing (referred to as OV later on) during core He burning on the expected period spectrum of ZZ Ceti stars, and also on the asteroseismologically derived parameters of ZZ Ceti stars.

In particular, the number of TPs experienced by the WD progenitor star through the AGB phase is quite uncertain and depends on the rate at which mass is lost during the TP-AGB phase, on the initial metallicity of the progenitor star, and the occurrence of extra mixing in the pulse-driven convection zone. To quantify the impact of all these uncertainties on the pulsational properties of ZZ Ceti stars, De Gerónimo et al. (2017) explored the situation in which the progenitor is forced to abandon the AGB phase before the occurrence of the first TP (0TP case) and also the case in which it experiences three TPs (3TP case). The results are shown in the left panels of Fig. 6, in which the chemical profiles (upper panel) for ${ }^{16} \mathrm{O},{ }^{12} \mathrm{C}$, and ${ }^{4} \mathrm{He}$, and the logarithm 
of the squared Brunt-Väisälä frequency (lower panel) in terms of the outer mass, for a DA WD model with $M_{\star}=0.548 M_{\odot}, T_{\text {eff }} \sim 12000 \mathrm{~K}$, and $M_{\mathrm{H}} \sim 4 \times 10^{-6} M_{\star}$, are displayed. In the $3 \mathrm{TP}$ case, there is an inter-shell region rich in ${ }^{4} \mathrm{He}$ and ${ }^{12} \mathrm{C}$ at the bottom of the ${ }^{4} \mathrm{He}$ buffer (see Fig. 2). At variance with this, in the 0TP situation no inter-shell region is expected. The presence or absence of an inter-shell region has a non-negligible impact on the Brunt-Väisälä frequency, and consequently on the period spectrum. Specifically, the resulting changes in the periods (at fixed radial order, $k$ ) are on average between 5 and $10 \mathrm{~s}$. In the case of more massive models, the average differences reduce to $\sim 2-3 \mathrm{~s}$. On the other hand, the amount of OV (central panel of Fig. 6) and different assumptions about the ${ }^{12} \mathrm{C}(\alpha, \gamma){ }^{16} \mathrm{O}$ reaction rate during core He burning (right panels of Fig. 6) impact to a lesser extent the pulsation periods, with average period differences of about $5 \mathrm{~s}$ when account is made of the current uncertainties in OV prescription and ${ }^{12} \mathrm{C}(\alpha, \gamma){ }^{16} \mathrm{O}$ reaction rate (Kunz et al. 2002).

The impact of the chemical structure built up during the TP-AGB evolution and the ${ }^{12} \mathrm{C}(\alpha, \gamma){ }^{16} \mathrm{O}$ reaction rate on the stellar parameters inferred from asteroseismological period-to-period fits of ZZ Ceti stars was studied by De Gerónimo et al. (2018). These authors found that the occurrence or not of TPs during AGB evolution implies an average deviation in the asteroseismological effective temperature $\left(\sigma_{T_{\text {eff }}}\right)$ of ZZ Ceti stars of at most $8 \%$, and of the order of $5 \%$ in the stellar mass $\left(\sigma_{M_{\star}}\right)$. For the mass of the $\mathrm{H}$ envelope, they find deviations up to 2 orders of magnitude — although generally much lower. These trends remain even when a sample of real ZZ Ceti stars is considered in the analysis. Noteworthy, the mean deviations in $T_{\mathrm{eff}}, M_{\star}$ and $M_{\mathrm{H}}$ inflicted by the uncertainties in the ${ }^{12} \mathrm{C}(\alpha, \gamma){ }^{16} \mathrm{O}$ reaction rate and $\mathrm{OV}$ are smaller than those produced by the uncertainties in the number of TPs.

In summary, the uncertainties in the parameters of the asteroseismological models due to uncertainties in the chemical profiles of WDs during the evolution of the progenitor stars are admittedly not negligible. However, the impact of the uncertainties on the chemical structure over the asteroseismological determinations is quantifiable and bounded, with average values close to the observational errors of the effective temperature and surface gravity. These results add confidence to the use of fully evolutionary models with consistent chemical profiles in WD asteroseismology, and render this approach as a robust way to peer into the internal structure of WD stars.

As a corollary of this Section, the two asteroseismological approaches commonly used in pulsating WDs described above are very different in nature, but complementary to each other. On the one hand, the parametric approach needs as a starting point certain constraints about the shape of the chemical profiles, the physically plausible chemical abundances in each part of the star $\left(X_{i}\right)$, and the specific chemical species that make up a WD $\left({ }^{1} \mathrm{H},{ }^{4} \mathrm{He},{ }^{12} \mathrm{C},{ }^{16} \mathrm{O}\right.$, etc $)$. This information is provided by the approach that uses detailed evolutionary models of WDs because in this treatment, the chemical profiles are consistent with the previous evolutionary history. At the same time, the parametric approach leads to seismological models with characteristics that differ somewhat from the canonical models of WDs, this way providing certain hints that can help to improve the evolutionary calculations. In other words, the parametric approach is useless if it does not take into account some robust constraints predicted by stellar evolution for the structure of a WD, and on the other hand, the approach using evolutionary models cannot ignore the apparently discordant results that the parametric approach predicts 
when it is applied to real stars. We conclude that it would be very important that efforts were combined in both methods in order to make progress in the detailed knowledge of the evolutionary origin and internal structure of WDs.

\subsection{Massive and ultra-massive WDs}

Another relevant progress in the field of WD asteroseismology in the last decade concerns the massive and ultra-massive WDs, which are key to understand physical processes in the AGB phase, the theory of crystallization, high-field magnetic WDs, and type Ia supernova explosions. These stars, which cluster around well-defined peaks in the WD mass distribution at $M_{\star} \sim 0.8 M_{\odot}$ and $M_{\star} \sim 1.10 M_{\odot}$, have been reported in several studies (see, e.g., Kepler et al. 2016b). Massive WDs $\left(0.8 \lesssim M_{\star} / M_{\odot} \lesssim\right.$ 1.0) are expected to have $\mathrm{C} / \mathrm{O}$ cores, with progenitor stars of $\sim 3-7 M_{\odot}$, while ultra-massive WDs $\left(M_{\star} / M_{\odot} \gtrsim 1.0\right)$ would result either from the evolution of single progenitors with masses in the range $\sim 7-8.5 M_{\odot}$, and characterized by $\mathrm{O} / \mathrm{Ne}$ cores, or from the merger of two $\sim 0.5-0.6 M_{\odot}$ WDs, and hence harboring $\mathrm{C} / \mathrm{O}$ cores (García-Berro et al. 2012). The internal structure and chemical stratification of massive and ultra-massive WDs can be, in principle, probed through asteroseismology. Fortunately, in recent years, a significant number of massive and ultra-massive ZZ Ceti stars have been uncovered (Kanaan et al. 2005; Castanheira et al. 2010, 2013; Hermes et al. 2013b; Curd et al. 2017). The ultra-massive ZZ Ceti star BPM 37093 (Kanaan et al. 1992, 2005) was the first object of this kind to be studied in detail, opening the opportunity to test crystallization theory (e.g., van Horn 1968) through asteroseismology (Metcalfe et al. 2004; Brassard and Fontaine 2005).

Asteroseismological studies based on fully evolutionary models (see Sect. 3.1) have been done for samples of massive and ultra-massive ZZ Ceti stars. For massive WDs, Romero et al. (2013) have been able to place constraints on the process of crystallization and phase separation of $\mathrm{C} / \mathrm{O}$ mixtures by using the phase diagrams of Segretain and Chabrier (1993) and Horowitz et al. (2010). In line with this, Althaus et al. (2012) found that, for a given value of $M_{\star}$, the amount of matter redistributed by phase separation is smaller when the Horowitz et al. (2010) phase diagram is considered instead of the Segretain and Chabrier (1993) one, leading to a smaller energy release from the process of $\mathrm{C} / \mathrm{O}$ separation. Regarding ultra-massive WDs, Camisassa et al. (2019) have presented detailed evolutionary sequences of ONe-core DA and DB WD models with stellar masses in the range $1.10-1.29 M_{\odot}$ extracted from the full evolution of 9-10.5 $M_{\odot}$ single progenitors from the ZAMS through core $\mathrm{H}$ and $\mathrm{He}$ stable burning and semi-degenerate $\mathrm{C}$ burning during the thermally pulsing Super-AGB (SAGB) phase (Siess 2010). In this way, realistic and consistent chemical profiles for the resulting WDs can be obtained, including the $\mathrm{O} / \mathrm{Ne}$ inner profiles and the outer chemical stratification, in particular the mass of the He inter-shell built up during the SAGB, a key issue as far as the assessment of cooling times at low luminosities is concerned. But the most novel aspect of these ultra-massive WD models, relevant for both evolutionary and pulsational inferences, is that the release of energy and the ensuing core chemical redistribution resulting from the phase separation of ${ }^{16} \mathrm{O}$ and ${ }^{20} \mathrm{Ne}$ upon crystallization (Medin and Cumming 2010) is included for the first time, 


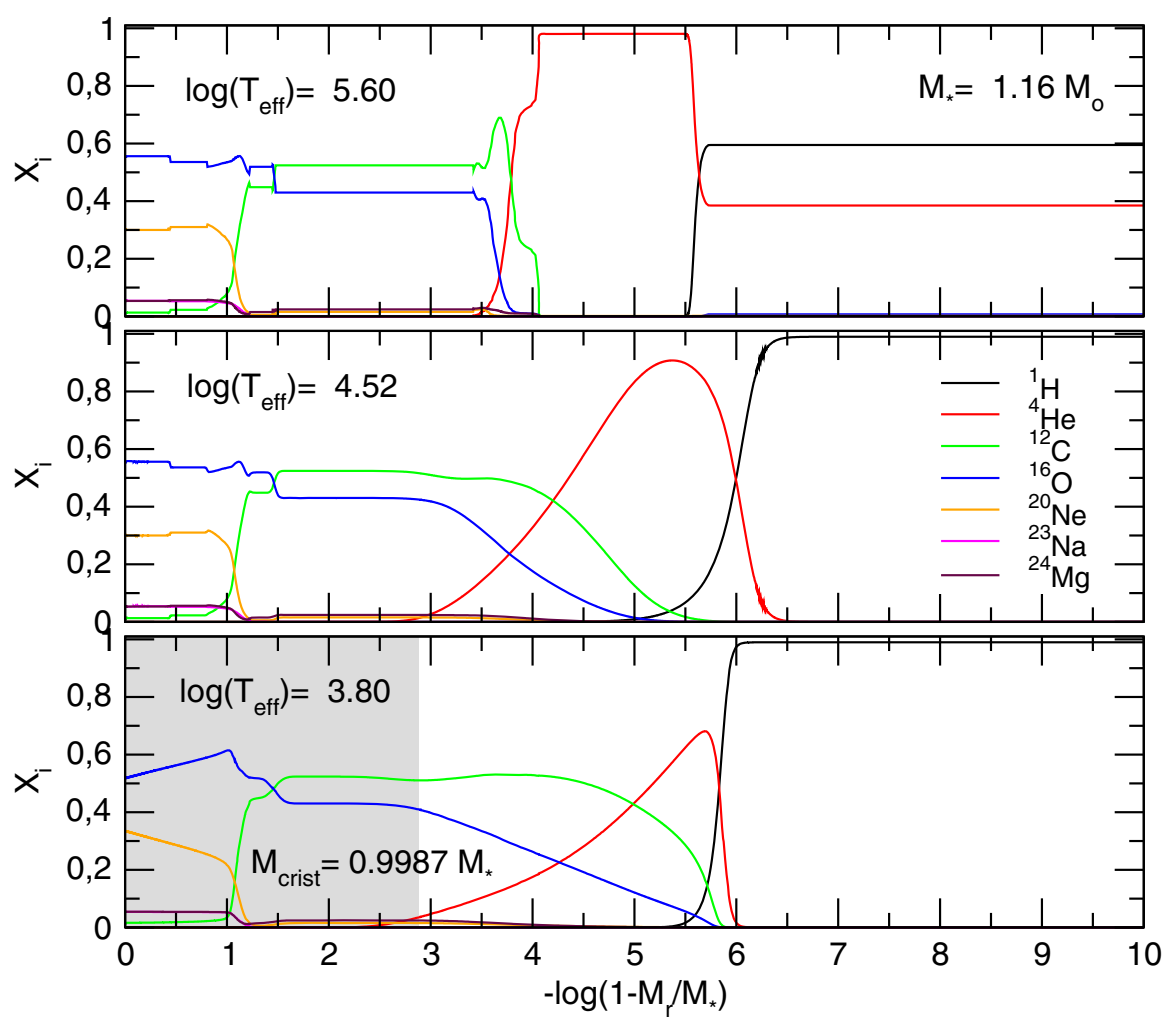

Fig. 7 Internal chemical profiles for $1.16 M_{\odot}$ DA WD models at three specific values of $\log \left(T_{\text {eff }}\right)$, as indicated at the left of each panel. The gray area in the lower panel emphasizes the crystallized region of the model, that comprises $99.87 \%$ of the total mass

thus substantially improving previous attempts at modelling these stars (Córsico et al. 2004; Althaus et al. 2007).

As shown by Camisassa et al. (2019), element diffusion strongly modifies the inner chemical distribution of ultra-massive WDs from the very early stages of WD evolution, as illustrated in Fig. 7 for selected $1.16 M_{\odot}$ models. Note that the heavy species are depleted from the outer layers as a result of gravitational settling and that the initial ${ }^{4} \mathrm{He}$ and ${ }^{12} \mathrm{C}$ distribution in the deep envelope results drastically modified. This is in contrast to the situation of average-mass WDs. These changes in the ${ }^{4} \mathrm{He}$ and ${ }^{12} \mathrm{C}$ profiles affect the radiative opacity in the envelope and thus the cooling times at late stages. The core chemical distribution is also strongly modified during the WD cooling due to phase separation during crystallization. It can be appreciated from the bottom panel of the figure, that corresponds to a WD model with $T_{\text {eff }} \sim 6300 \mathrm{~K}$. Note that most of the star should be crystallized (99.87\%, gray zone).

The phase diagram for ${ }^{16} \mathrm{O}-{ }^{20} \mathrm{Ne}$ plasmas predicts that all ultra-massive $\mathrm{ONe}$-core DAVs reported in the literature with masses higher than $1.10 M_{\odot}$ should have more than $80 \%$ of their mass crystallized (Camisassa et al. 2019). This is borne out in Fig. 8, in which the evolutionary tracks for ultra-massive WDs with $M_{\star}=1.10,1.16,1.22$, 


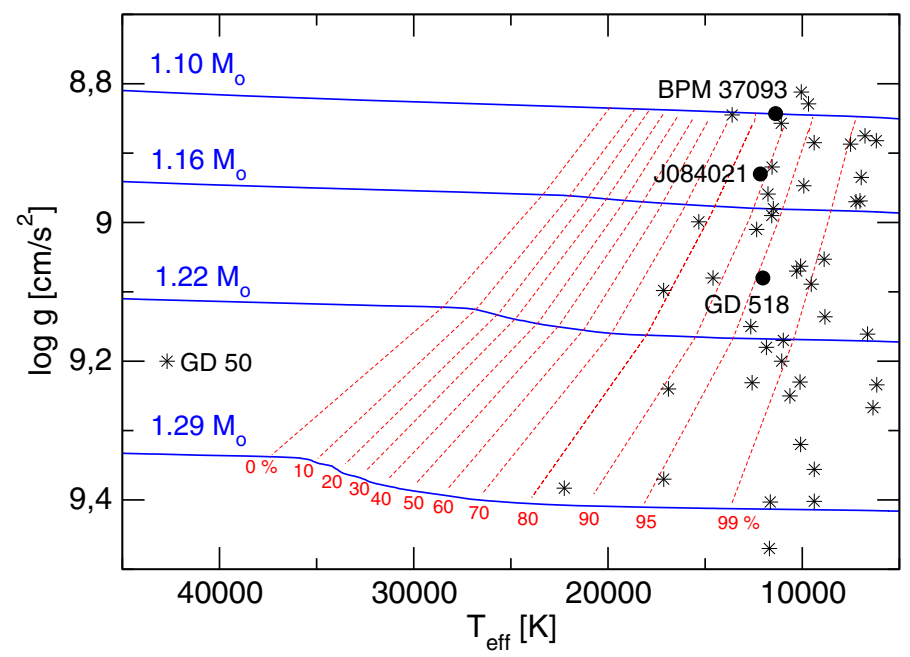

Fig. 8 Evolutionary tracks of the ultra-massive DA WD models computed by Camisassa et al. (2019) in the plane $T_{\text {eff }}-\log g$. Red dashed lines indicate $0,10,20,30,40,50,60,70,80,90,95$ and $99 \%$ of crystallized mass. The location of ultra-massive DA WD stars (Kleinman et al. 2013; Kepler et al. 2016b; Curd et al. $2017)$ is indicated with black-star symbols. The black circles indicate the location of the known ultramassive ZZ Ceti stars: BPM 37093 (Nitta et al. 2016), J084021 (Curd et al. 2017), and GD 518 (Hermes et al. 2013a)

and $1.29 M_{\odot}$ in the $T_{\text {eff }}-\log g$ diagram is displayed. The increase in the surface gravity for all of the models at the onset of crystallization is due to a decrease in the stellar radius caused by the changes in the abundance distribution of ${ }^{16} \mathrm{O}$ and ${ }^{20} \mathrm{Ne}$ at the core, induced by phase separation during crystallization. Also plotted in Fig. 8 is the location of spectroscopically identified ultra-massive WDs (asterisk symbols). It is apparent that a large fraction of stars in this sample should have more than $99 \%$ of their mass crystallized. We also include three known ultra-massive ZZ Ceti stars: BPM 37093, J084021, and GD 518. Note that, in particular, BPM 37093 should have $\sim 88 \%$ of its mass in a crystalline state, whereas the more massive stars, J084021 and GD 518, should be $\sim 90 \%$ and $\sim 96 \%$ crystallized.

Asteroseismology constitutes a very promising way to infer and test the occurrence of crystallization in WD interiors as well as physical processes related with dense plasmas. A first step in this direction has been given by De Gerónimo et al. (2019a), who explored the pulsational properties - mainly the period spectrum and the distribution of the period spacings - of the ultra-massive DA WDs models of Camisassa et al. (2019), taking into account the impact of crystallization on the $g$-mode period spectrum through the hard-sphere boundary condition (Montgomery and Winget 1999). In Fig. 9 , we plot the chemical profiles and the logarithm of the squared Brunt-Väisälä and Lamb frequencies as a function of the fractional mass for an ONe-core WD model with $M_{\star}=1.16 M_{\odot}, T_{\text {eff }} \sim 11500 \mathrm{~K}$, and a percentage of crystallized mass of $\sim 93 \%$. For this model, which is located at the middle of the ZZ Ceti instability strip, phase separation due to crystallization - which shapes the ${ }^{16} \mathrm{O}$ and ${ }^{20} \mathrm{Ne}$ chemical profileshas already finished. De Gerónimo et al. (2019a) discuss the possibility of discerning 


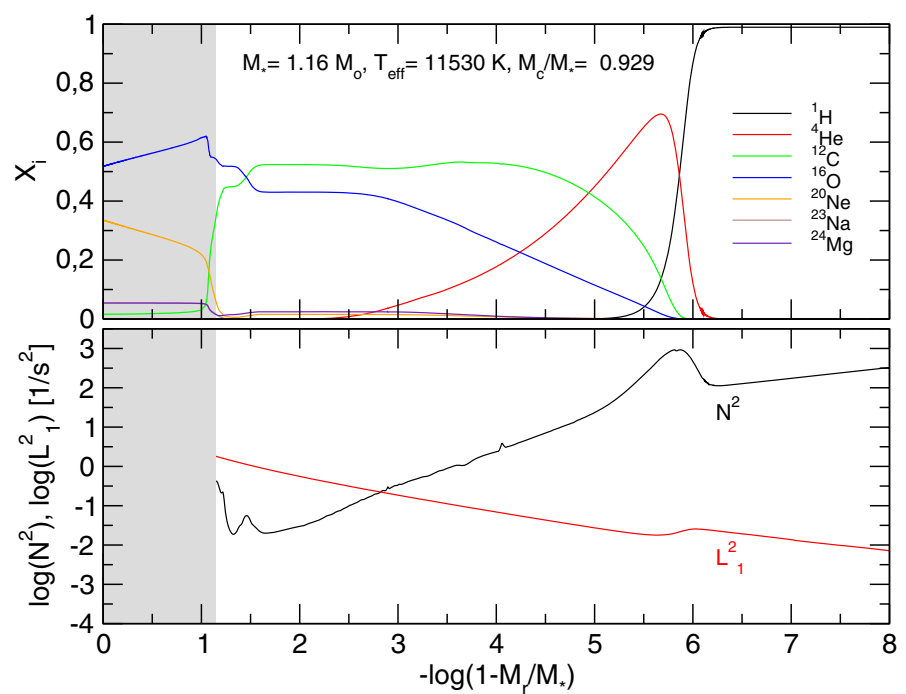

Fig. 9 Abundances by mass of ${ }^{1} \mathrm{H},{ }^{4} \mathrm{He},{ }^{12} \mathrm{C},{ }^{16} \mathrm{O},{ }^{20} \mathrm{Ne},{ }^{23} \mathrm{Na}$, and ${ }^{24} \mathrm{Mg}$ (upper panel) and the logarithm of the squared Brunt-Vïsälä and Lamb (with $\ell=1$ ) frequencies (lower panel) as a function of the fractional mass, corresponding to an ONe-core WD model with $M_{\star}=1.16 M_{\odot}$ and $T_{\text {eff }} \sim 11500 \mathrm{~K}$. The model has been computed taking into account latent heat release and chemical redistribution due to phase separation during crystallization. The gray area marks the domain of crystallization. $M_{\mathrm{c}} / M_{\star}$ is the fraction of the crystallized mass of the model

whether an ultra-massive $\mathrm{ZZ}$ Ceti star has a core made of ${ }^{12} \mathrm{C}$ and ${ }^{16} \mathrm{O}$ or a nucleus of ${ }^{16} \mathrm{O}$ and ${ }^{20} \mathrm{Ne}$ on the basis of period-spacing diagrams. They find that period spacing departures due to mode trapping effects are weaker for ONe-core WD models than for CO-core WD models with the same effective temperature and stellar mass (Fig. 10). These authors conclude that the features found in the period-spacing diagrams could be used as a seismological tool to discern the core composition of ultra-massive ZZ Ceti stars, something that should be complemented with detailed asteroseismic analysis using the individual observed periods.

Independent research on the evolution of ultra-massive WDs has been performed by Lauffer et al. (2018), who used MESA (Paxton et al. 2011, 2013, 2015, 2018, 2019) to compute evolutionary sequences of H- and He-rich atmosphere WD models with masses in the range $1.012-1.307 M_{\odot}$. These authors present chemical profiles for the whole mass range considered, covering different core chemical compositions (i.e. $\mathrm{CO}, \mathrm{ONe}$ and $\mathrm{NeOMg}$ ) and its dependence on the stellar mass. In addition, they present the initial-to-final mass relation, the mass-radius relation and cooling times considering the effects of the atmosphere and the core composition. At variance with Camisassa et al. (2019), however, the models of Lauffer et al. (2018) do not take into account the phase separation and the consequent core chemical rehomogeneization during crystallization. Also, the predicted compositions of the WD cores differ from those of Ritossa et al. (1996) and Siess (2010). Differences can be traced back to the extreme choice of macrophysics parameters done in Lauffer et al. (2018), particularly the choice of a large convective boundary mixing $f$ value below the C-burning flame 

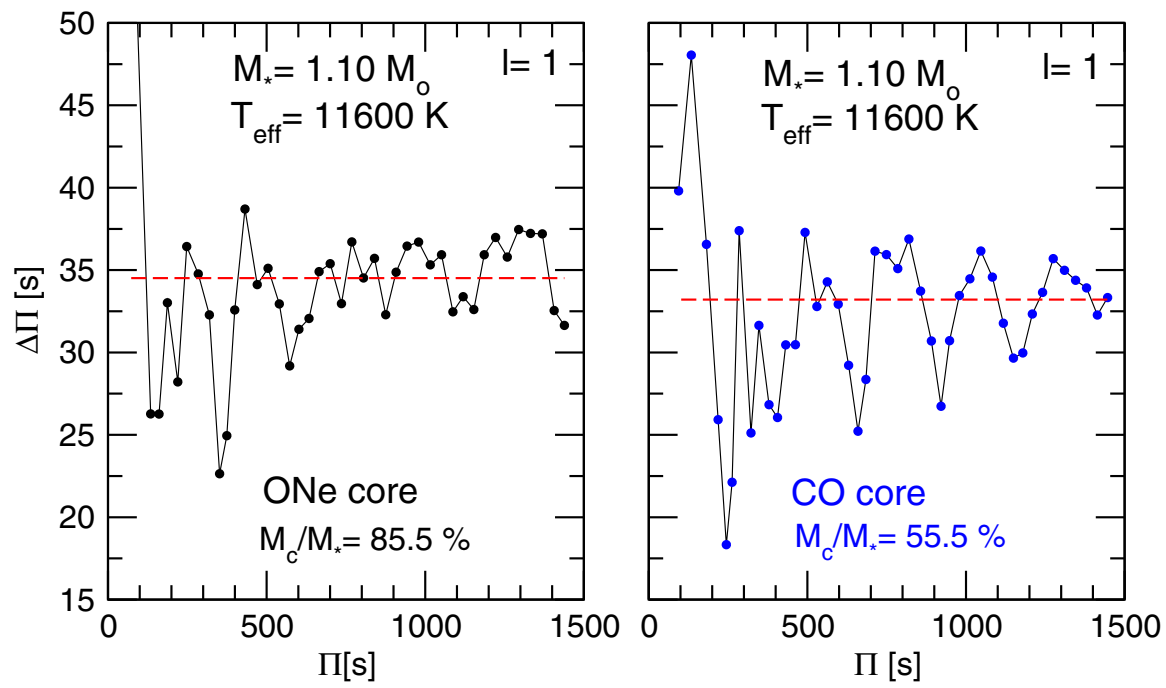

Fig. 10 Forward period spacing $(\Delta \Pi)$ in terms of the periods of $\ell=1$ pulsation $g$ modes for $1.10 M_{\odot}$ WD models at $T_{\text {eff }} \sim 11600 \mathrm{~K}$ with an ONe core (left panel) and a CO core (right panel). In both models, latent-heat release and chemical redistribution caused by phase separation have been taken into account during crystallization. The percentages of the crystallized mass are indicated. The horizontal red-dashed line is the asymptotic period spacing

$(f=0.1)$, which is at variance with expectations from hydrodynamical simulations (Lecoanet et al. 2016). Also, another source of discrepancy that may have an important impact on the composition and properties of the WD models is the extreme mass-loss rates on the SAGB phase assumed in Lauffer et al. (2018) (rates of Bloecker 1995, with $\eta=10$ ). Regarding this last issue, due to the extreme SAGB winds adopted in their modeling, stellar models avoided the thermally pulsing SAGB phase that shapes the outer regions of the ultra-massive WD cores. These are particularly important points with regard to the pulsational properties of ultra-massive DA WDs.

\subsection{Excitation mechanisms}

The early non-adiabatic works on the excitation of ZZ Ceti pulsations (Dolez and Vauclair 1981; Dziembowski and Koester 1981; Winget et al. 1982b) assumed that $g$ mode periods are much shorter than the turn-over time of convection of a ZZ Ceti star $\left(\tau_{\text {conv }} \gg \Pi_{g}\right)$ and neglected the perturbation of the convective flux $\left(\delta \mathbf{F}_{c}\right)$ in the pulsation equations - the so-called "frozen-convection" approximation (FC; Unno et al. 1989). Under this assumption, these authors found that the physical agent responsible for the driving of $g$-mode pulsations at the hot boundary (blue edge) of the ZZ Ceti instability strip should be the $\kappa-\gamma$ mechanism acting at the $\mathrm{H}$ partial ionization zone ${ }^{12}$. However, Brickhill (1991) realized that the turn-over timescale in the convection zone of a ZZ Ceti star, at least at the blue edge, is shorter than the pulsation periods of inter-

$\overline{12}$ Actually, Dziembowski and Koester (1981) found $g$-mode instability due to the partial ionization of He. 
Table 11 Observed and theoretical effective temperature (in K) of the edges of the ZZ Ceti and V777 Her instability strip

\begin{tabular}{lllll}
\hline & $\begin{array}{l}\text { Blue edge } \\
\text { ZZ Ceti }\end{array}$ & $\begin{array}{l}\text { Red edge } \\
\text { ZZ Ceti }\end{array}$ & $\begin{array}{l}\text { Blue edge } \\
\text { V777 Her }\end{array}$ & $\begin{array}{l}\text { Red edge } \\
\text { V777 Her }\end{array}$ \\
\hline Observed & $\sim 12500$ & $\sim 10800$ & $\sim 31100$ & $\sim 23000$ \\
Theoretical, TDC & 11970 & 5600 & 30000 & 13000 \\
Theoretical, FC & 11750 & 5520 & 28000 & 13000 \\
\hline
\end{tabular}

The theoretical values are obtained for $0.6 M_{\odot}$ CO-core WD models

est ( $\tau_{\text {conv }} \ll \Pi_{g}$ ), that is, the exact opposite of the FC approximation. Brickhill (1991) (see also Goldreich and Wu 1999) proposed the "convective-driving" mechanism as the responsible for pulsations in ZZ Ceti stars. Under this hypothesis, the convective flux should react instantaneously to the macroscopic motions of pulsations (Brassard and Fontaine 1997, 1999).

In an effort to include consistently the impact of possible interactions between convection and pulsations on the precise location of the blue and red edges of the ZZ Ceti instability strip, Van Grootel et al. (2012) applied a time-dependent convection (TDC) treatment in the framework of the mixing-length theory (Grigahcène et al. 2005) for the first time in WDs, thus avoiding in their stability computations the extreme assumptions adopted in previous approaches. These authors also performed nonadiabatic computations assuming the FC approximation for comparison. The results of this work-summarized in Table 11-are a bit surprising. Indeed, the predicted boundaries of the ZZ Ceti instability strip according to the TDC treatment are not much different from those obtained with the FC approach. Specifically, the effective temperature of the blue edge derived from the TDC treatment is only $\sim 240 \mathrm{~K}$ higher than that obtained with the FC approximation, and in good agreement with the instantaneous-convection adaptation results of Brassard and Fontaine (1997, 1999). In the case of the red edge, the TDC approach predicts an effective temperature barely higher $(\sim 80 \mathrm{~K})$ than the FC approach does, but both fail to match the observed red edge of the ZZ Ceti instability strip. Similar results to those described for the ZZ Ceti stars using the TDC and FC treatments were obtained for V777 Her stars ( Dupret et al. 2008; Quirion et al. 2008; Van Grootel et al. 2017, see Table 11). All these results indicate that the interaction between pulsations and convection does not seem to be relevant in ZZ Ceti and V777 Her pulsating stars in terms of the location of the edges of their instability strips.

Recently, Luan and Goldreich (2018) showed that $g$ modes of pulsating DA WDs with angular frequency $\omega<L_{\ell, b}\left(L_{\ell, b}\right.$ being the Lamb frequency at the base of the surface convection zone) suffer enhanced radiative damping that exceeds convective driving rendering them damped, thus giving origin to the red edge of the ZZ Ceti instability strip. These authors also give an explanation for the sporadic outbursts exhibited by some ZZ Ceti stars observed by the Kepler mission that are located near the red edge of the instability strip (See Sect. 3.4).

A potentially efficient mechanism of mode excitation in DA WD stars is the $\epsilon$ mechanism, in which pulsational instability is induced by thermonuclear reactions 
(Unno et al. 1989). In this mechanism, the driving is due to the strong dependence of nuclear burning on temperature. Usually, WDs are considered to lack nuclear burning. In the case of DA WDs, this is true in general, but in some circumstances these stars may sustain substantial nuclear burning even at low luminosities, and in particular, during the ZZ Ceti stage. This has been explored by Althaus et al. (2015) in the case of DA WDs coming from low-metallicity progenitors. These authors found that for progenitor metallicities $Z$ in the range $0.00003-0.001$, and in the absence of third dredge-up during the TP-AGB phase (and the ensuing carbon enrichment of the envelope), the resulting $\mathrm{H}$ envelope of the average-mass DA WDs is thick enough to make stable $\mathrm{H}$ burning the most important energy source even at low luminosities. This finding has been exploited by Camisassa et al. (2016) who have investigated the possible excitation of $g$ modes in ZZ Ceti stars due to the $\epsilon$ mechanism by H burning. They demonstrated that, for WDs with masses smaller than $\sim 0.70 M_{\odot}$ and effective temperatures lower than $11600 \mathrm{~K}$ that evolved from low-metallicity progenitors, the dipole and quadrupole $g$ modes with radial order $k=1\left(g_{1}\right)$ are excited mostly as a result of the H-burning shell through the $\epsilon$ mechanism. However, the $\epsilon$ mechanism is insufficient to drive these modes in WDs descended from solar-metallicity progenitors. These results encourage the possibility of placing constraints on $\mathrm{H}$ shell burning in cool WDs and the efficiency of third dredge-up episodes during the preceding AGB phase if the $g_{1}$ mode were detected in DA WDs associated with low-metallicity environments, such as globular clusters and/or the Galactic halo.

Finally, it is worth mentioning the possible existence of very hot pulsating DA pre-WDs with $g$ modes excited by the $\epsilon$ mechanism ${ }^{13}$. Early work on this issue by Kawaler (1988) revealed pulsation instability in models of H shell-burning planetary nebula nuclei. Later, Charpinet et al. (1997) predicted $g$-mode instability promoted by $\mathrm{H}$ burning through the $\epsilon$ mechanism in post-EHB stars. These pioneering researches were extended by Maeda and Shibahashi (2014), who found that nuclear reactions in the $\mathrm{H}$ burning shell can drive low-degree $g$ modes with periods in the range 40-200 s for DA pre-WDs with $40000 \leq T_{\text {eff }} \leq 300000 \mathrm{~K}$ coming from solarmetallicity progenitors, the instability domain in the HR diagram being sensitive to the $\mathrm{H}$ content. Similar results, although on the basis of DA pre-WD models coming from low-metallicity progenitors (Althaus et al. 2015), have been found by Calcaferro et al. (2017c). In Fig. 11, we show the instability domain of hot DA pre-WDs with low-order $g$ modes excited by the $\epsilon$ mechanism on the $\log T_{\text {eff }}-\log g$ plane. The boundaries of the instability domain for different sub-solar metallicities computed by Calcaferro et al. (2017c) are depicted with thick lines of different colors and styles. We also include the results of Maeda and Shibahashi (2014) for solar metallicity. Star symbols show the location of DAO and hot DA stars that could be candidate targets to be scrutinized to search for pulsations. The finding of any of these stars to be pulsating with short-period $g$ modes could allow to place constraints on the H thickness of DA WDs (Maeda and Shibahashi 2014).

An alternative possibility for driving pulsations in WDs could be tidal excitation of $g$ modes in compact WD binary systems due to resonances between the orbital

\footnotetext{
13 Pulsational excitation of $g$ modes due to the $\epsilon$ mechanism in H-deficient pre-WD models was investigated by Kawaler et al. (1986) and Córsico et al. (2009b) (see, e.g., Althaus et al. 2010b; Catelan and Smith 2015, for a review of this topic).
} 


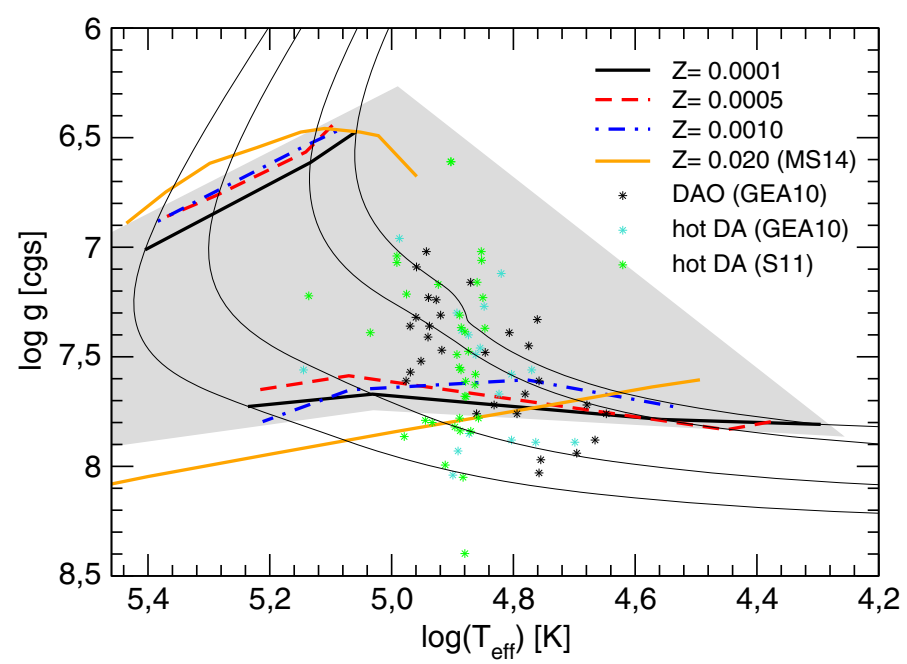

Fig. 11 Instability domain (shaded area) of hot DA pre-WDs with low-order $g$ modes excited by the $\epsilon$ mechanism on the $\log T_{\text {eff }}-\log g$ plane. Thick lines show the edges of the instability region for different progenitor metallicities. Also included are the results of Maeda and Shibahashi (2014) (MS14) for solar metallicity. Star symbols of different colors show the DAO and hot DA stars from Gianninas et al. (2010) (GEA10) and hot DA stars from Sion (2011) (S11). Black lines depict evolutionary tracks corresponding to $M_{\star}=0.535,0.568,0.666,0.738 M_{\odot}$ and $Z=0.0001$

frequency and the discrete spectrum of eigenfrequencies of the WDs. These tidally forced $g$-mode oscillations have been thoroughly investigated by Fuller and Lai (2011, 2012). They showed that the excited $g$ modes can reach very large amplitudes at the regions close to the stellar surface, where they are likely dissipated through a combination of non-linear processes and radiative damping, thus probably preventing the excitation of discrete $g$ modes. In any case, no tidally excited $g$ modes have been detected in any WD so far (see Kilic et al. 2018).

We close this section by describing the possible existence of pulsating DA WDs somewhat hotter than ZZ Ceti stars, with effective temperatures comparable to those of the DBV WDs. Winget et al. (1982b) (see, also, Winget 1982) were the first to find $g$-mode pulsational instability due to the partial ionisation of He in models of DA WDs harboring very thin $\mathrm{H}$ envelopes $\left(M_{\mathrm{H}} / M_{\star} \lesssim 10^{-10}\right)$, for effective temperatures of $19000 \mathrm{~K}$. On the other hand, Kurtz et al. (2013) announced the existence of a new class of DA WD pulsators, the so-called pulsating hot DAV WDs, characterized by $T_{\text {eff }} \sim$ $30000 \mathrm{~K}$, that show $g$ modes probably excited by a driving mechanism very different than those described above. The driving mechanism for hot DAVs was formulated by Shibahashi $(2005,2007)$ (see, also, Shibahashi 2013) who theoretically found that in DA WDs with thin $\mathrm{H}$ envelopes at the cool edge of the 45000-30000 K effective temperature range (the "DB gap"), a super-adiabatic, chemically inhomogeneous $(\mu$ gradient) zone drives pulsation $g$ modes with high $\ell$ values, but even with some $\ell<3$ modes excited, which could be observable. Three objects of this predicted class are known, the characteristics of which are summarized in Table 12, although the observations should be confirmed with additional monitoring. Further investigation of 
Table 12 Properties of the known Hot DAV stars (adapted from Bognár and Sódor 2016)

\begin{tabular}{llllll}
\hline Star & $T_{\text {eff }}[\mathrm{K}]$ & $\log g[\mathrm{cgs}]$ & $V[\mathrm{mag}]$ & $\Pi[\mathrm{s}]$ & References \\
\hline WD 0101+145 & 29980 & 7.38 & $18.8(\mathrm{~g})$ & 159 & Kurtz et al. (2008) \\
WD 0232-097A & 30110 & 7.30 & $17.8(\mathrm{~g})$ & 705 & Kurtz et al. (2008) \\
WD 1017-138 & 32600 & 7.80 & 14.6 & 624 & Kurtz et al. (2013) \\
\hline
\end{tabular}

these stars could open the chance of a direct test of the explanation/existence of the DB gap through asteroseismology.

\subsection{Outbursting pulsating DAV stars}

Kepler spacecraft observations of ZZ Ceti stars revealed a new type of phenomena never before observed from the ground: outburst-like events in DAV stars with effective temperatures near the red edge of the instability strip (Bell et al. 2017b) ${ }^{14}$. The first ZZ Ceti star exhibiting outbursts was WD J1916+3938 (KIC4552982). This star was the first ZZ Ceti identified in the original Kepler mission field (Hermes et al. 2011). After 1.5 years of observations which provided the longest pseudo-continuous light curve ever recorded for a ZZ Ceti, Bell et al. (2015) found that the star shows a rich period spectrum typical of DAVs, with at least 20 modes detected, along with a total of 178 enhancements of brightness typical of outburst phenomena, with peaks of up to $17 \%$ above the quiescent level and involving very energetic events $\left(\sim 10^{33} \mathrm{erg}\right)$ with a mean recurrence period of about 2.7 days. Remarkably, KIC4552982 is located at the cool boundary of the ZZ Ceti instability strip $\left(T_{\text {eff }}=10,860 \mathrm{~K}, \log g=8.16\right)$. The second ZZ Ceti star showing outbursts was PG1149+057 (Hermes et al. 2015), discovered with the K2 mission. This star exhibits flux enhancements of up to $45 \%$. Being relatively bright, it was possible to determine for this ZZ Ceti that the outbursts actually affect the normal pulsations, thus demonstrating that outbursts are an intrinsic phenomenon of the star. At present, a total of eight outbursting ZZ Ceti stars have been discovered ( Bell et al. 2016, 2017b; Bell 2017). In Fig. 12 we depict the ZZ Ceti instability strip in the $T_{\text {eff }}-\log g$ diagram with the location of the known DAVs, emphasizing the outbursting objects with red square symbols. All of the outbursting ZZ Ceti stars share some common properties: (i) the outbursts can enhance the mean flux of the star over timescales of hours, with an irregular recurrence on timescales of days; (ii) the normal ZZ Ceti-like $g$-mode pulsation spectrum changes in amplitude and frequency during outbursts, relative to those in quiescence; and (iii) the effective temperatures of outbursting ZZ Cetis locate them close to the cool edge of the instability strip, suggesting that outbursts could be related to the cessation of $g$-mode pulsations (Bell et al. 2017b).

A physical explanation for the occurrence of outbursts in cool ZZ Ceti stars was suggested by Hermes et al. (2015) and formally drawn up by Luan and Goldreich (2018),

\footnotetext{
14 A phenomenon reminiscent of these outburst-like events was the sforzando event detected in 1996 for the DBV GD358, in which the star dramatically altered its pulsation characteristics on a timescale of hours (Provencal et al. 2009).
} 


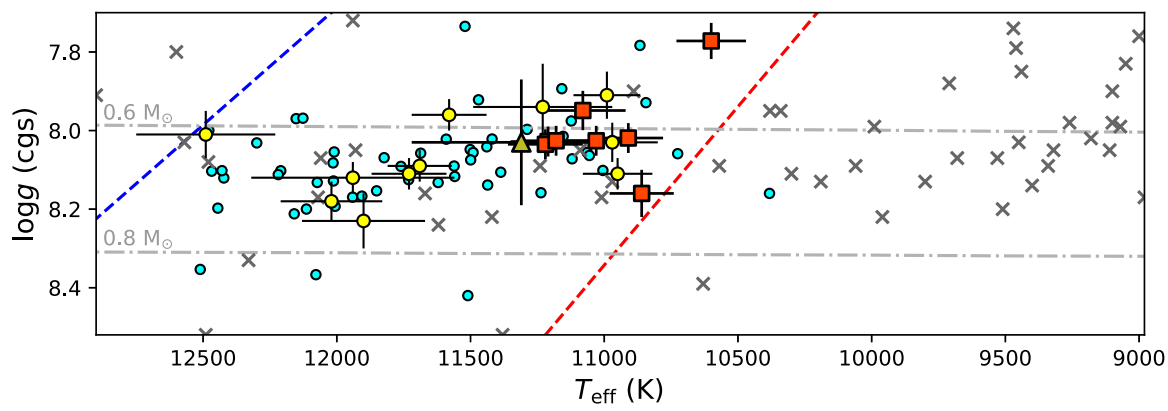

Fig. $12 T_{\text {eff }}-\log g$ diagram showing the location of presently known outbursting DAVs (8 objects) marked with red squares. The dark-yellow triangle corresponds to the object EPIC 211891315 from Bell et al. (2016), that exhibited a single outburst-type event. The small yellow circles and gray crosses correspond to the DAVs and long-cadence WD targets that do not show outbursts in K2 data through Campaign 6 (Bell et al. 2016). The dashed lines are the observational boundaries of the ZZ Ceti instability strip, according to Tremblay et al. (2015). Dash-dotted lines are WD cooling tracks for $M_{\star}=0.6 M_{\odot}$ and $M_{\star}=0.8 M_{\odot}$ WDs from Fontaine et al. (2001). The cyan circles correspond to the location of DAVs known from the ground (Gianninas et al. 2011), including SDSS J2350-0054 (Mukadam et al. 2004; Kepler et al. 2016b) that is cooler than the red edge of the instability strip (Figure adapted from Bell 2017)

in terms of parametric instability via mode coupling of WD pulsations (Dziembowski 1982; Wu and Goldreich 2001). In this scenario, it is possible that the oscillation kinetic energy of a parent mode (or multiple parent modes) grows linearly until it reaches a critical value. When this value is exceeded, the mode enters a nonlinear regime and rapidly transfers its energy to resonant daughter modes, or a cascade of resonant daughter modes, which may be quickly damped by turbulence in the convection zone. The sum of the frequencies of the daughter modes must be close to the parent frequency. The energy of these modes is deposited at the basis of the outer convection zone, producing the sudden enhancement of the stellar brightness characterizing the outbursts. Luan and Goldreich (2018) attribute ZZ Ceti outbursts to limit cycles arising from sufficiently resonant 3-mode couplings between overstable parent modes and pairs of radiatively damped daughter modes. Limit cycles account for the durations and energies of outbursts $\left(\sim 10^{33}-10^{34} \mathrm{erg}\right)$ and their prevalence near the cool edge of the ZZ Ceti instability strip.

\subsection{ELMV and pre-ELMV stars}

One of the most important findings of recent years in the field of variable WDs is the discovery of pulsations in DA low-mass (LM) and extremely low-mass (ELM) WDs and pre-WDs. DA WDs have a mass distribution that peaks at $0.59 M_{\odot}$, but it also shows a peak at low masses: $M_{\star} / M_{\odot} \lesssim 0.45$ (Kleinman et al. 2013; Kepler et al. 2015). LM WDs can be the result of enhanced mass loss before the occurrence of the He flash during the red giant branch phase of low-mass stars. At variance with average WDs with $\mathrm{C} / \mathrm{O}$ cores, these stars are expected to harbor cores made of $\mathrm{He}$, since $\mathrm{He}$ ignition is avoided. In particular, strong mass loss resulting from interactive binary evolution is needed to explain the origin of the so-called ELM WDs, which have 
masses below $\sim 0.18-0.20 M_{\odot}{ }^{15}$ (see Sect. 2.2 and Althaus et al. 2013; Istrate et al. 2016b, for details). In Fig. 13 we show the evolutionary tracks of Althaus et al. (2013) for He-core LM and ELM WDs in the $T_{\text {eff }}-\log g$ diagram. Sequences with masses in the range $0.18-0.20 \lesssim M_{\star} / M_{\odot} \lesssim 0.4$ experience multiple CNO-cycle flashes during the early-cooling phase, which leads to the intricate loops in the diagrams. In the last decade, numerous LM WDs, including ELM WDs, have been detected with the ELM Survey and the SPY and WASP surveys (see Koester et al. 2009; Brown et al. 2010, 2012, 2013, 2016, 2017b; Maxted et al. 2011; Kilic et al. 2011, 2012, 2015; Gianninas et al. 2014, 2015). We include in Fig. 13 the location of a sample of LM and ELM WDs and pre-WDs with the aim of illustrating the region in the $\log T_{\text {eff }}-\log g$ plane that is populated by these stars.

The possible existence of pulsations in ELM WDs was predicted by Steinfadt et al. (2010) for the first time, by scaling of the thermal timescale at the basis of the outer convective zone of their ELM WD models with surface gravity. They found that, contrary to what happens in the case of ZZ Ceti stars $\left(M_{\star} \gtrsim 0.50 M_{\odot}\right)$, in pulsating ELM WDs, the $g$-mode pulsations should probe the core regions of the WDs, by virtue that the eigenfunctions are not excluded from those regions due to the low degeneracy of ELM WDs. The long time (several Gyr) that ELM WDs should spend at low effective temperatures $\left(T_{\text {eff }} \lesssim 14000 \mathrm{~K}\right.$ ) due to vigorous stable $\mathrm{H}$ burning via the pp chain, motivated Steinfadt et al. (2012) to carry out a careful search for pulsating objects with masses $M_{\star} \lesssim 0.20 M_{\odot}$, but with null results. Shortly after, Hermes et al. (2012) reported the exciting discovery of the first pulsating ELM WD, SDSS $\mathrm{J} 184037.78+642312.3$, the coolest and the lowest-mass pulsating WD at that time, with $T_{\text {eff }}=9100 \pm 170 \mathrm{~K}, \log g=6.22 \pm 0.06$, and $M_{\star} \sim 0.17 M_{\odot}$. This pulsating ELM WD-which is in a $4.6 \mathrm{hr}$ binary system with another WD star-exhibits high-amplitude, multi-periodic variability with periods in the range $2000--4900$ $\mathrm{s}$, compatible with intermediate- and high-order $g$ modes. The increasing interest in pulsating LM and ELM WDs led to the discovery of more objects of this type. At present, eleven pulsating WDs of this kind are known (Hermes et al. 2012, 2013d, b; Kilic et al. 2015, 2018; Bell et al. 2017a, 2018; Pelisoli et al. 2018b). This number includes all the known and suspected ELMV stars, that is, objects that show radial velocity $(\mathrm{RV})$ variations confirming the binary nature expected for He-core WDs, and objects that do not ${ }^{16}$. This new class of variable stars has been designed as ELMV. Note that both the pulsating LM WDs $\left(0.18-0.20 \lesssim M_{\star} / M_{\odot} \lesssim 0.45\right)$ and the pulsating ELM WDs $\left(M_{\star} / M_{\odot} \lesssim 0.18-0.20\right)$ are designated by the common name of ELMVs. They are listed in Table 7, along with updated effective temperatures and

15 The definition of an ELM WD is still under debate. In the context of the ELM Survey (Brown et al. 2010), an ELM WD is defined as an object with surface gravity of $5 \lesssim \log g \lesssim 7$ and effective temperature in the range of $8000 \lesssim T_{\text {eff }} \lesssim 22000 \mathrm{~K}$ (see, e.g., Brown et al. 2016). Here (see also Córsico and Althaus 2014a), we propose to define an ELM WD as a WD that does not undergo $\mathrm{H}$ shell flashes, because in this way, the pulsational properties are quite different as compared with the systems that experience flashes, although this mass limit depends on the metallicity of the progenitor stars (Serenelli et al. 2002; Istrate et al. 2016b).

16 According to Kilic et al. (2018), there are only four confirmed pulsating ELM WDs in short-period binaries (which are the four that show RV variations), that occupy a similar parameter space and there is no question about their nature as WDs. These are: SDSS J1112+1117, SDSS J1518+0658, SDSS J1840+6423, and PSR J1738+0333. We have to add SDSSJ1618+3854 to that list, based on Bell et al. (2018). 
A. H. Córsico et al.

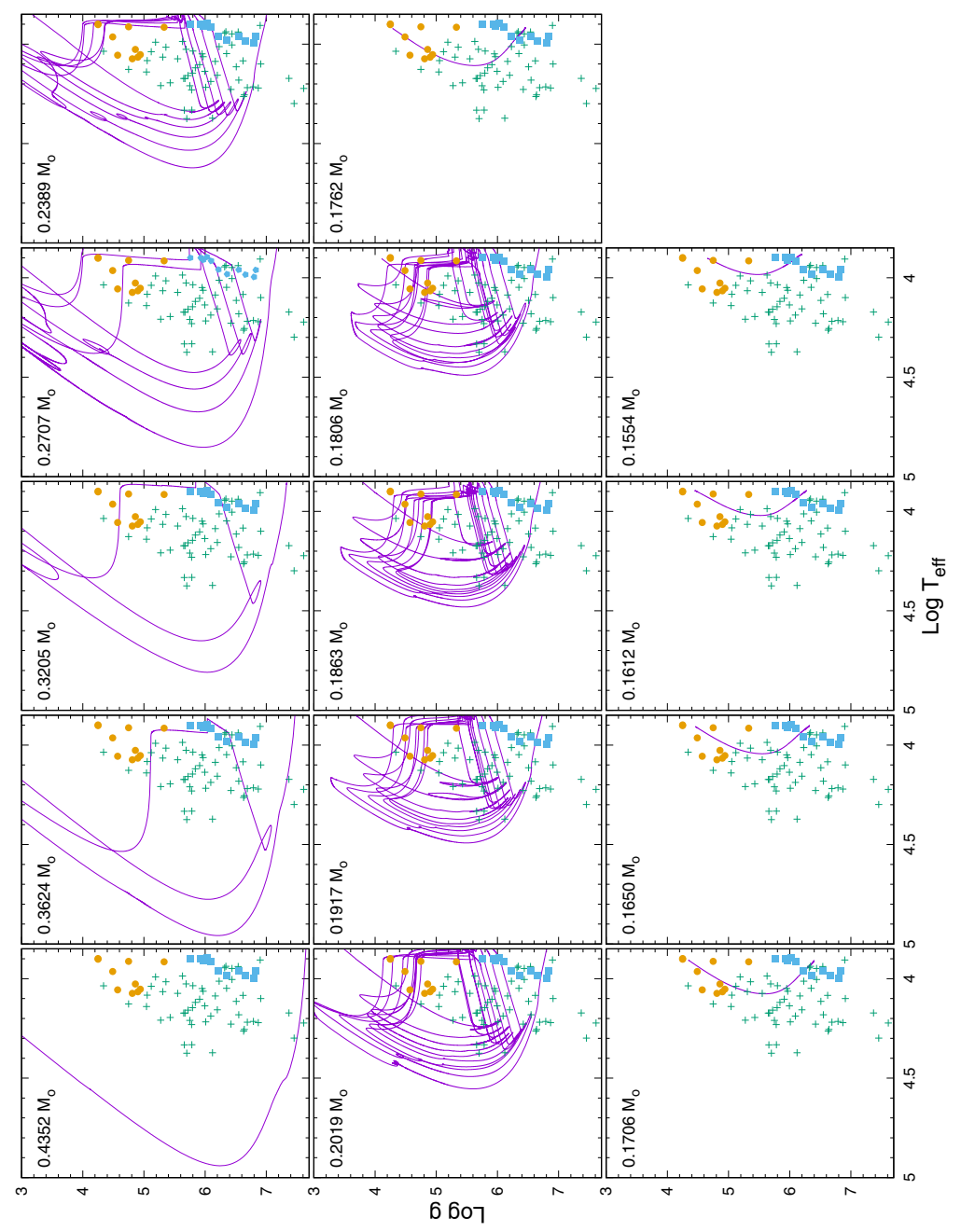

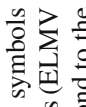

色兘

远非

的芯

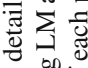

t.

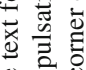

\%

is

路

임

产的

힝ㅎㅎ

¿ํㅡㅇ

त्

跣

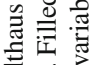

的文

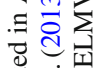

เ

ठํ

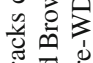

范

총

흥ㅎㅁㅍㅚ

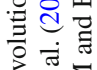

ठั

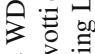

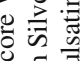

话

I

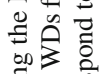

垫

卷完

氙

要焉品

$\infty \geq$ 的

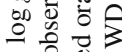

1 을

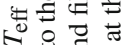

응 흥

$m$ क्षे

운 웡 营 


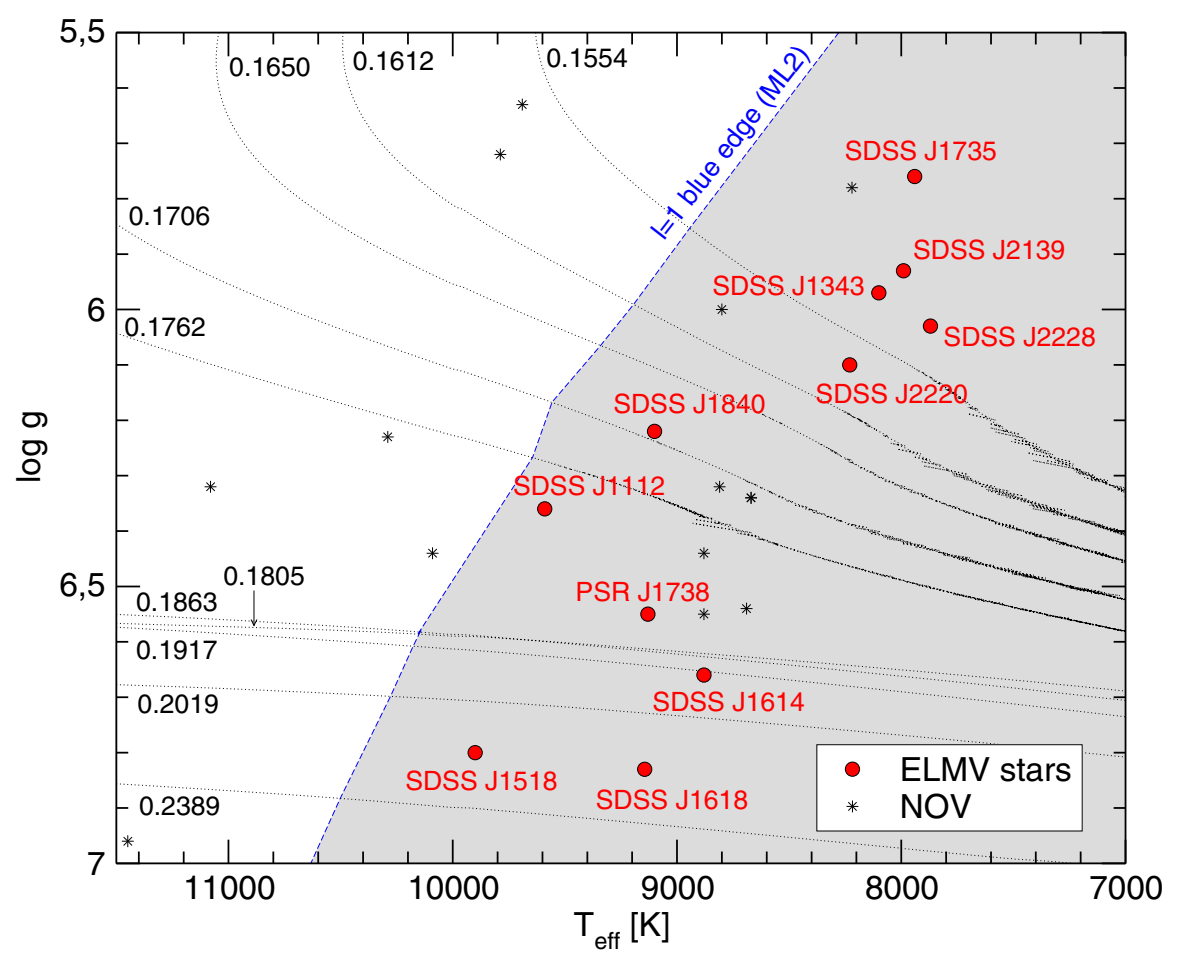

Fig. $14 T_{\text {eff }}-\log g$ diagram showing the location of the eleven known ELMVs, marked with red circles ( $T_{\text {eff }}$ and $\log g$ computed with 1D model atmospheres after 3D corrections). Black-star symbols are objects not observed to vary (NOV). Dotted lines correspond to the low-mass He-core WD evolutionary tracks (final cooling branches) of Althaus et al. (2013). The "spikes" exhibited by the tracks with $M_{\star} \leq 0.1762 M_{\odot}$ are the result of intense $\mathrm{H}$ residual burning ( $p p$ chain) at low $T_{\text {eff values. The gray region limited by the dashed }}$ blue line corresponds to the instability domain of $\ell=1 \mathrm{~g}$ modes due to the $\kappa-\gamma$ mechanism acting at the $\mathrm{H}--\mathrm{H}^{+}$partial ionization region, according to Córsico and Althaus (2016)

gravities (3D corrected; Tremblay et al. 2015), magnitudes, and period ranges. We emphasize that Table 7 includes all-the known and suspected-ELMV stars.

The existence of ELMV stars (red circles in Fig. 14) constitutes an unprecedented opportunity for probing their subsurface layers and ultimately to place constraints on the currently accepted formation scenarios by means of asteroseismology. Indeed, the importance of these stars has motivated intensive theoretical work to explore the basic nature of their pulsations (Steinfadt et al. 2010; Córsico et al. 2012d; Van Grootel et al. 2013; Córsico and Althaus 2014a, b, 2016; Calcaferro et al. 2017a, b, 2018b). In line with the pioneering work by Steinfadt et al. (2010), Córsico and Althaus (2014a) concluded that for ELM WDs, $g$ modes mainly probe the core regions, while $p$ and radial modes (if excited) sound the stellar envelope. Hence, pulsations in ELMVs offer the rare opportunity - in the context of pulsating WDs - to put constraints to both the core and envelope chemical structure of these stars by means of asteroseismology. In Fig. 15, we display the chemical profiles (upper panel) and the propagation diagram (lower panel), for a representative ELM WD model characterized by $M_{\star}=0.1554 M_{\odot}$ 


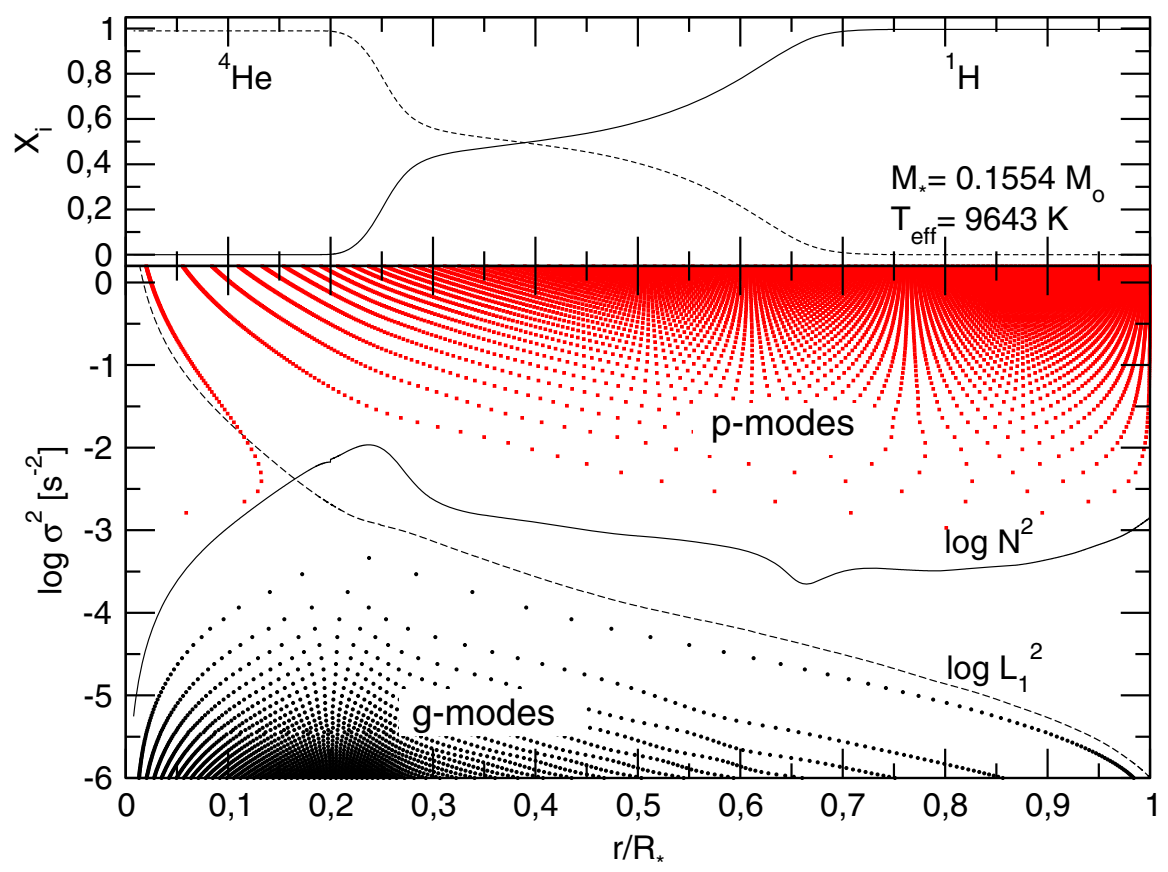

Fig. 15 Fractional abundances of $\mathrm{He}$ and $\mathrm{H}$ as a function of the normalized radius (upper panel), and a propagation diagram (lower panel) corresponding to a representative ELM WD model with $M_{\star}=0.1554 M_{\odot}$ and $T_{\text {eff }} \sim 9600 \mathrm{~K}$. Small squares (in red) correspond to the spatial location of the nodes of the radial eigenfunction of $\ell=1 p$ modes, whereas small circles (in black) represent the location of the nodes of dipole $g$ modes. Solid (dashed) black curve corresponds to the logarithm of the squared Brunt-Väisälä (Lamb) frequency

and $T_{\text {eff }} \sim 9600 \mathrm{~K}$. Figure 16 shows the density of kinetic energy of oscillation $\left(d E_{\text {kin }} / d r\right.$, normalized to 1$)$ for dipole $(\ell=1) g$ and $p$ modes with different values of the radial order $k$, for the same ELM WD model. The fact that the Brunt-Väisälä frequency adopts large values in the core (Fig. 15) allows eigenfunctions of $g$ modes to penetrate to those deep regions of the star, where most of the kinetic energy of oscillation is concentrated (Fig. 16). On the other hand, in LM WDs, $g$ modes are very sensitive to the $\mathrm{He} / \mathrm{H}$ compositional gradient (see Fig. 8 of Córsico and Althaus 2014a) and then, they could be employed as a diagnostic tool for constraining the $\mathrm{H}$ envelope thickness, similar to ZZ Ceti stars.

Another important finding in this area is that time-dependent element diffusion markedly modifies the shape of the $\mathrm{He} / \mathrm{H}$ chemical transition region, and in turn, has a strong impact on the $g$-mode pulsation spectrum of ELM WDs ( Córsico et al. 2012d; Córsico and Althaus 2014a). The effects are weaker-but still non-negligible-for LM WDs. Therefore, it is apparent that time-dependent element diffusion must be taken into account in any pulsational study of ELMV stars.

A detailed linear pulsation-stability analysis of ELMV stars has been carried out by Van Grootel et al. (2013). These authors employed a sophisticated treatment of TDC in their nonadiabatic pulsation computations, similar to that applied by the same authors 


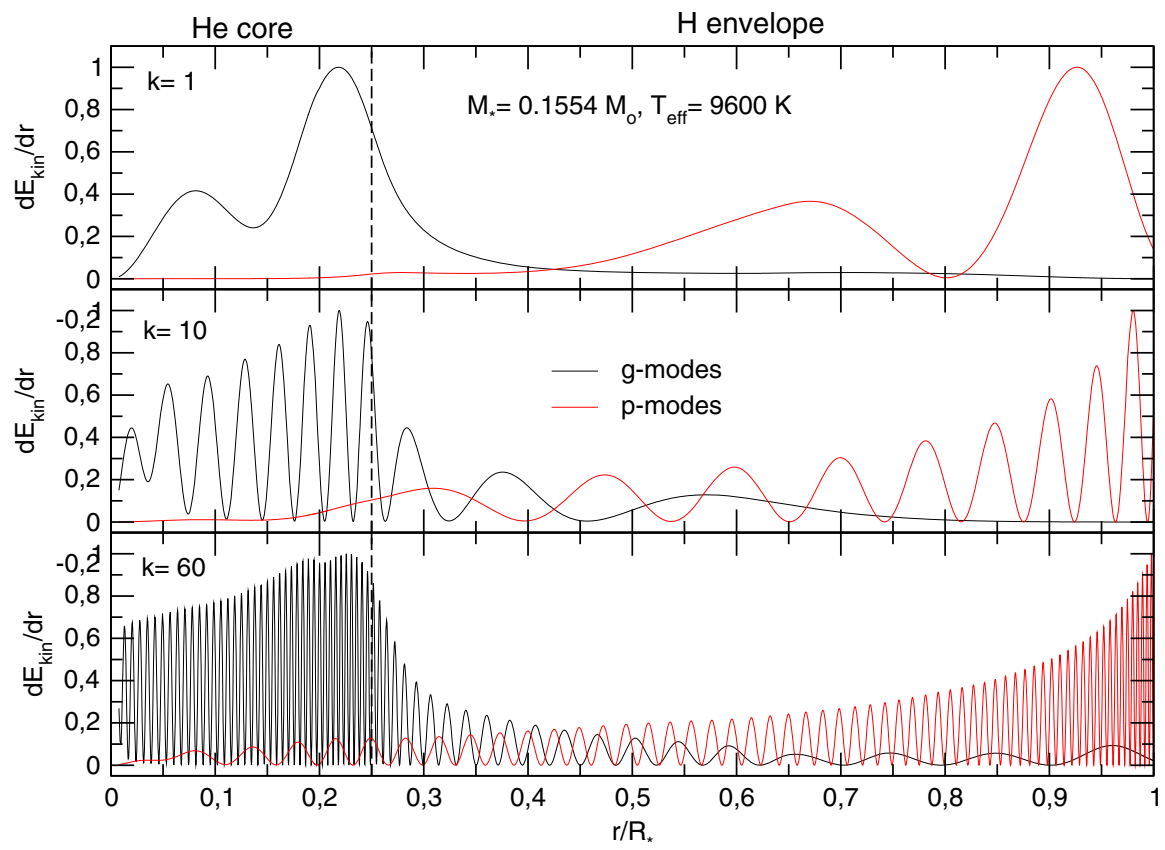

Fig. 16 Run of the density of oscillation kinetic energy for dipole $p$ modes (red) and $g$ modes (black) with $k=1$ (upper panel), $k=10$ (middle panel), and $k=60$ (lower panel), in terms of the normalized radius, corresponding to the same ELM WD model shown in Fig. 15. The vertical dashed line marks the approximate location of the $\mathrm{He} / \mathrm{H}$ chemical transition region

to ZZ Ceti and V777 Her stars (see Van Grootel et al. 2012, 2017, and Sect. 3.4). The excitation mechanism is the convective-driving mechanism. They obtained numerous excited $g$ modes with periods in a range that depends on the stellar mass. For instance, for a $0.2 M_{\odot}$ model, the range of periods of the excited modes is $\sim 500$ to $8000 \mathrm{~s}$. Their computations, based on envelope models, predict a blue edge of the ELMV instability domain in good agreement with the observations. However, as for ZZ Ceti and V777 Her stars, their calculations are unable to account for the cool edge, which according to their predictions should be located at $T_{\text {eff }} \sim 5500 \mathrm{~K}$, i.e., much cooler than the observed one ( $\left.T_{\text {eff }} \sim 7900 \mathrm{~K}\right)$. Instead, Van Grootel et al. (2013) estimate the location of the red edge by requiring that the thermal timescale in the driving region at the base of the $\mathrm{H}$ convection zone be equal to the critical period beyond which $\ell=1$ $g$ modes cease to exist. This estimation is based on the atmosphere energy-leakage argument elaborated by Hansen et al. (1985). This estimate indicates a theoretical red edge close to the observed one; however, it is not completely satisfactory, because several ELMVs are cooler than the red edge predicted in that way. Another relevant result, that is in line with the previous findings of these authors for the ZZ Ceti and V777 Her stars, is that the blue edge of the instability domain of ELMVs does not change substantially if the FC approach is used instead of the more physically sound TDC treatment. Again, it seems that the interaction between pulsation and convection 
does not dramatically affect the position in the $\log T_{\text {eff }}-\log g$ diagram at which the LM and ELM WDs begin to pulsate.

Córsico and Althaus (2014b, 2016) have independently explored in detail the instability strip of ELMV stars, based on the fully evolutionary low-mass He-core WD models with H-pure envelopes of Althaus et al. (2013), and assuming the FC approximation for their nonadiabatic computations. In particular, Córsico and Althaus (2016) took into account three different prescriptions for the MLT theory of convection and covered a wide range of effective temperatures and stellar masses. For each model, the pulsation stability of radial $(\ell=0)$ and nonradial $(\ell=1,2) p$ and $g$ modes was assessed. Their main predictions are illustrated in Fig. 14, which shows the instability domain of $\ell=1 \mathrm{~g}$ modes in the spectroscopic HR diagram due to the $\kappa-\gamma$ mechanism acting at the $\mathrm{H}-\mathrm{H}^{+}$partial ionization region, together with some selected low-mass He-core WD evolutionary tracks (final cooling branches), and the location of ELMVs (red circles). The instability domain is emphasized by a gray region bounded by a dashed blue line corresponding to the blue edge of the instability domain. Notably, the FC results of Córsico and Althaus (2016) are in good agreement with the TDC results of Van Grootel et al. (2013) in terms of the location of the blue edge of the ELMV instability strip. Similar to Van Grootel et al. (2013), Córsico and Althaus (2016) did not find a red edge of the instability strip consistent with the observed one. It is found that the blue edge of the instability domain in the $T_{\text {eff }}-\log g$ plane is hotter for higher stellar mass and larger convective efficiency and that the blue edge of $p$ modes does not depend on the harmonic degree. However, in the case of $g$ modes, a weak sensitivity of the blue edge with $\ell$ is found. In addition, the blue edges corresponding to radial and nonradial $p$ modes are almost coincident with each other, and somewhat hotter $(\sim 200 \mathrm{~K})$ than the blue edges of $g$ modes. Finally, we emphasize that some short-period $g$ modes can be destabilized mainly by the $\varepsilon$ mechanism due to stable nuclear burning at the basis of the H envelope, particularly for model sequences with $M_{\star} \lesssim 0.18 M_{\odot}$ (see Córsico and Althaus 2014b, 2016; Fontaine et al. 2017, for details).

It is worth mentioning that the ranges of unstable-mode periods predicted by current stability analyses are in excellent agreement with the ranges of periods observed in the ELMV stars (see, for instance, Figs. 10 to 17 of Córsico and Althaus 2016). However, for all the analyzed ELMVs, the number of periods detected is alarmingly low in comparison with the rich spectrum of periods of radial and nonradial $p$ and $g$ unstable modes expected from theoretical stability analyses. Similar to other kinds of pulsating WDs, it is suspected that an unknown mechanism should be at work in real stars that favors only a few modes (out of the available dense spectrum of eigenmodes) to reach observable amplitudes. Finding that missing piece of physics in the pulsation models is a challenge for future research.

Apart from ELMVs, multi-periodic pulsations in five objects that are supposed to be precursors of LM and ELM WDs have been detected in the last few years (Maxted et al. 2013, 2014b; Gianninas et al. 2016). These stars, that typically have $8000 \lesssim T_{\text {eff }} \lesssim 13000 \mathrm{~K}$ and $4 \lesssim \log g \lesssim 5.5$, are represented in Fig. 17 as blue circles. They show a surface composition made of a mixture of $\mathrm{H}$ and $\mathrm{He}$, suggesting that some mechanism must be delaying (or even inhibiting) the effects of chemical diffusion. They are called pre-ELMV stars and constitute a new class of pulsating stars. 


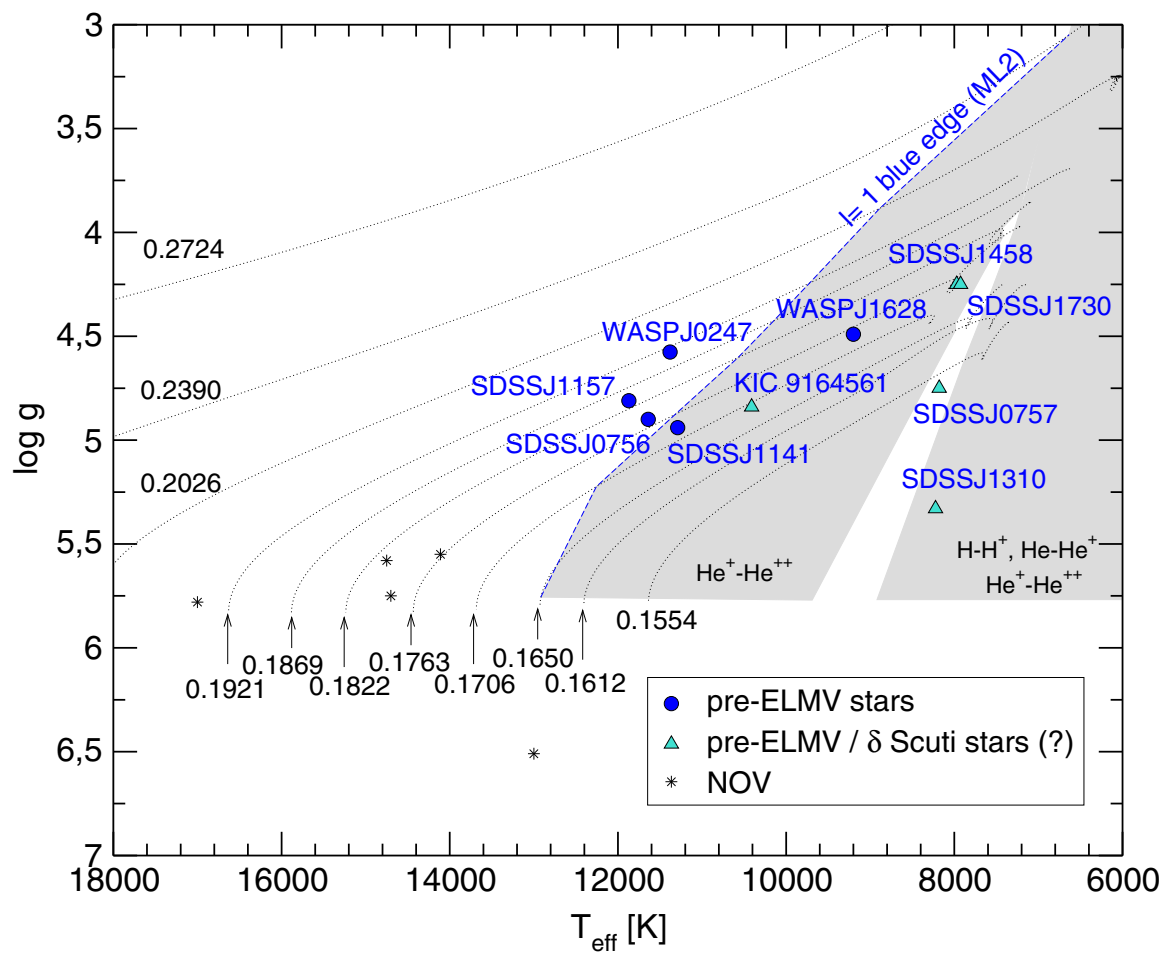

Fig. $17 T_{\text {eff }}-\log g$ diagram showing low-mass He-core pre-WD evolutionary tracks (dotted curves) computed neglecting element diffusion. Numbers correspond to the stellar masses of each sequence. Blue circles correspond to the known pre-ELMV stars with a secure identification, turquoise triangles stand for objects that could be pre-ELMVs or $\delta$ Scuti/SX Phoenicis stars as well, and black-star symbols depict the location of stars not observed to vary (NOV). The dashed blue line indicates the nonradial dipole $(\ell=1)$ blue edge of the pre-ELMV instability domain (emphasized as a gray area) due to the $\kappa-\gamma$ mechanism acting at the $\mathrm{He}^{+}-\mathrm{He}^{++}$partial ionization region, as obtained by Córsico et al. (2016a)

Also, the discovery of long-period $(\Pi \sim 800-4700 \mathrm{~s})$ pulsations in five additional objects located at the same region of the $T_{\text {eff }}-\log g$ diagram has been reported. These stars are represented with turquoise triangles in Fig. 17. The exact nature of these pulsating stars is uncertain since they could be identified as pre-ELMVs or as SX Phe and/or $\delta$ Scuti pulsating stars as well. In Table 8 we include an updated compilation of the effective temperature, gravity, and range of observed periods for all the known pre-ELMV stars, including those with an indefinite identification. The data were extracted from Maxted et al. (2013, 2014b) and Gianninas et al. (2016) for the confirmed pre-ELMVs, and from Zhang et al. (2016), Corti et al. (2016), Bell et al. (2018) and Pelisoli et al. (2018b) for stars with ambiguous identification (pre-ELMVs or $\delta$ Scuti/SX Phoenicis stars).

The first theoretical study exploring the pulsation stability properties of radial modes of static low-mass He-core pre-WD models was that of Jeffery and Saio (2013). They were successful in identifying the instability boundaries associated with radial modes characterized by low-to-high radial orders, and showed that they are very sensitive 
to the chemical composition at the driving region. In particular, these authors found that the modes are excited by the $\kappa-\gamma$ mechanism operating mainly in the second He ionization zone $\left(\mathrm{He}^{+}-\mathrm{He}^{++}\right)$, provided that the driving region is depleted in H. Córsico et al. (2016a) extended the work of Jeffery and Saio (2013) by analyzing the pulsational stability of radial and nonradial $p$ and $g$ modes with periods in the range $10 \mathrm{~s} \lesssim \Pi \lesssim 20000 \mathrm{~s}$, on the basis of He-core, low-mass pre-WD evolutionary models. In Fig. 17, we show with a dashed blue line the nonradial $\ell=1$ blue edge of the pre-ELMV instability domain (gray area) due to the $\kappa-\gamma$ mechanism acting at the $\mathrm{He}^{+}-\mathrm{He}^{++}$partial ionization region ( $\log T \sim 4.7$ ). Non-diffusion low-mass He-core pre-WD evolutionary tracks are displayed with dotted curves. The results of Córsico et al. (2016a) for radial modes (not shown) are in good agreement with the predictions of Jeffery and Saio (2013). At $T_{\text {eff }} \lesssim 7800 \mathrm{~K}$ there is also some mode driving due to the $\mathrm{He}-\mathrm{He}^{+}$and $\mathrm{H}-\mathrm{H}^{+}$partial ionization zones $(\log T \sim 4.42$ and $\log T \sim 4.15$, respectively). The blue edge for $\ell=2$ modes (not shown) is slightly ( $\sim 10-30 \mathrm{~K}$ ) hotter than the $\ell=1$ blue edge. The location of the blue edges does not depend on the prescription for the MLT theory of convection adopted in the equilibrium models, at variance with what happens in the case of ELMVs. The blue edge of radial modes is substantially cooler $(\sim 1000 \mathrm{~K})$ than for nonradial modes. The nature of the pulsation modes excited in pre-ELMV stars has been established as being highfrequency $p$ modes and intermediate-frequency "mixed" $p$ - $g$ modes (Scuflaire 1974). These modes behave like $g$ modes in the inner parts of the star and like $p$ modes in the outer parts. This is due to the very peculiar shape of the Brunt-Väisälä frequency profile in the inner regions of the star (see Figs. 2 and 3 of Córsico et al. 2016a).

The theoretical computations of Córsico et al. (2016a) account for the existence of some of the known pre-ELMVs, including the observed ranges of excited periods. As an example, in Fig. 18 we show the case of WASP J1628+10B. In this figure, we depict the theoretical periods of unstable dipole modes in terms of $T_{\text {eff }}$ with the values of the logarithm of the $e$-folding time, corresponding to sequences with $M_{\star}=0.1706 M_{\odot}$ and $M_{\star}=0.1763 M_{\odot}$. The most unstable modes (smaller $e$-folding times) have the shorter periods. The pulsation periods of WASP J1628+10B are marked with horizontal segments. Note that the two periods of this star are well accounted for by the theoretical predictions at the right effective temperature. The computations of Córsico et al. (2016a), however, fail to predict pulsations in three pulsating objects: WASP0247, SDSSJ1157, and SDSS0756 (the hottest ones, see Fig. 17). This is intimately linked to the abundance of $\mathrm{He}$ at the driving zone; i.e., larger $\mathrm{He}$ abundances at the envelope of the models are required in order for the blue edge to be hotter, so as to include these three stars within the instability domain. This could be achieved by adopting different masses for the initial donor star in the original binary system. That could lead to low-mass pre-WD models with different He abundances at their envelopes.

The results described above are derived from computations in which element diffusion is not allowed to operate. When element diffusion is considered in the pre-ELM WD models, the driving region is quickly depleted of He by virtue of gravitational settling, and hence the instability region in the $T_{\text {eff }}-\log g$ plane shrinks. In this case, none of the known pre-ELMVs is within the instability region. This very important result strongly suggests that element diffusion should not be operative in the low-mass 


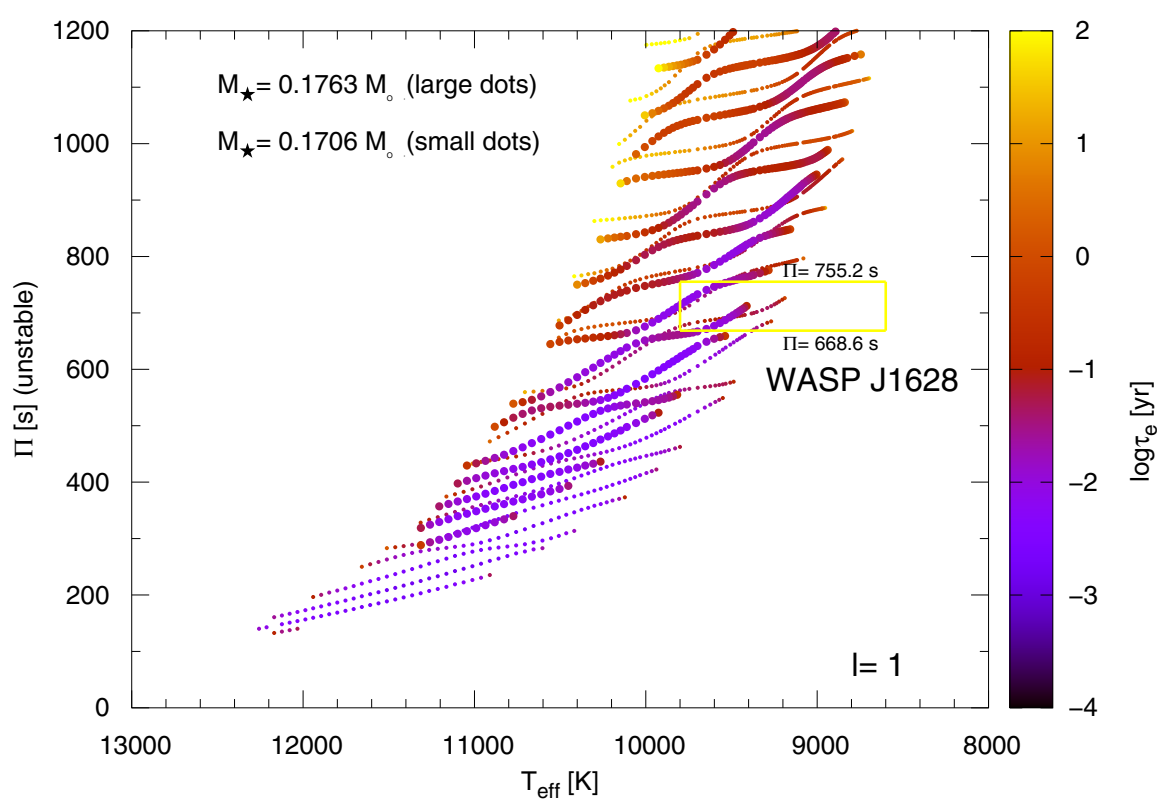

Fig. 18 Periods of unstable $\ell=1$ modes in terms of the effective temperature, with the palette of colors (right scale) indicating the value of the logarithm of the $e$-folding time (in years), corresponding to the sequences with $M_{\star}=0.1706 M_{\odot}$ (small dots) and $M_{\star}=0.1763 M_{\odot}$ (large dots) neglecting element diffusion. Also shown are the pulsation periods of the pre-ELM WD star WASP J1628+10B

pre-WD stage. This is a necessary condition also for pre-ELMVs to have envelopes with mixed $\mathrm{H}$ and $\mathrm{He}$, as observed (Gianninas et al. 2016). To prevent (or diminish) the effects of element diffusion, two main factors could be playing a role, namely stellar winds and/or stellar rotation. This interesting issue has been recently addressed by Istrate et al. (2016a), who demonstrated that rotational mixing, which counteracts gravitational settling, can maintain a sufficient amount of He within the driving region of the pre-ELM WDs. In this way, He can drive pulsational instabilities with periods that are in line with the observed periods. These authors point out that, by the time such a star enters its cooling track, gravitational settling overcomes rotational mixing, allowing the formation of a pure $\mathrm{H}$ envelope. At this point, the star is able to develop pulsation $g$ modes due to the partial ionization of $\mathrm{H}$, becoming an ELMV variable star. According to this scenario, it is expected that the ELMVs are rotating, and that rotation should be evidenced in splittings of the pulsation frequencies. However, no rotational-splitting signals have yet been detected in the pulsation spectrum of any ELMV star.

Very interesting research about the evolutionary and pulsational properties of WASP 0247-25B, a pre-ELMV star component of the double-lined eclipsing system WASP 0247-25, was presented by Istrate et al. (2007). Maxted et al. (2013) provided fundamental parameters of this star at a unique level of precision. By employing stateof-the-art evolutionary models that take into account rotational mixing and diffusion processes along with a comprehensive stability pulsation analysis, Istrate et al. (2007) 
were able to find a stellar model that closely reproduces observed properties of the star such as stellar mass, orbital period, surface gravity, effective temperature, surface chemical composition, and also the pulsation periods detected in the star. This reinforces the validity of the pre-ELM WD models of Istrate et al. (2016a) that incorporate rotational mixing.

An aspect of crucial importance in the investigation of low-mass WDs is the precise identification of the individual stars, either as precursor objects of WDs (pre-WDs) or as stars that have already entered their WD cooling tracks. According to theoretical models (Althaus et al. 2013; Istrate et al. 2016b), low-mass WDs cool more slowly than low-mass pre-WDs. On these grounds, it is expected that ELMVs should exhibit smaller rates of period change, $\dot{\Pi}(\equiv d \Pi / d t)$, than pre-ELMVs. Hence, the eventual measurement of the rate of period change for a given pulsating star in the region of the $\log T_{\text {eff }}-\log g$ diagram populated by ELMVs and pre-ELMVs could be potentially useful to know its evolutionary stage. Also, a measurement of $\dot{\Pi}$ could help to distinguish ELM WDs $\left(M_{\star} \lesssim 0.18-0.20 M_{\odot}\right)$, that have thick $\mathrm{H}$ envelopes and long cooling timescales, from LM WDs $\left(M_{\star} \gtrsim 0.18-0.20 M_{\odot}\right)$, characterized by thinner $\mathrm{H}$ envelopes and shorter cooling timescales. On the other hand, some of these stars may be headed towards a CNO-cycle flash, and thus have much larger — and more easily detectable- $\dot{\Pi}$ values. Also, in view of the systematic difficulties in the spectroscopic classification of stars of the ELM Survey (Bell et al. 2017a), an eventual measurement of $\dot{\Pi}$ could help to confirm that a given pulsating star is an authentic low-mass WD and not a star at a different evolutionary stage.

Calcaferro et al. (2017a) have carried out a comprehensive analysis of the secular rates of period change theoretically expected in ELMV and pre-ELMV stars, as well as WD precursors that are evolving at stages prior to the development of CNO-cycle flashes during the early cooling phase. For the case of ELMVs, we show in Fig. 19 the maximum absolute value expected for the rate of period change, $\left|\dot{\Pi}_{\max }\right|$, as a function of $M_{\star}$, for $\ell=1 g$ and $p$ modes, and also for radial modes $(\ell=0)$, for selected values of the radial order $k$, covering the range of periods typically observed in ELMV stars. In the case of $g$ modes, which are the modes that have been detected so far in these stars, ${ }^{17}$ there is a clear distinction in the magnitude of $\left|\dot{\Pi}_{\text {max }}\right|$ depending on whether $M_{\star} \lesssim 0.18 M_{\odot}$ or $M_{\star} \gtrsim 0.18 M_{\odot}$. Specifically, the rates of period change for ELM WDs are about ten times smaller than for more massive models. In contrast, for radial modes and $p$ modes, there is no clear differentiation in $\left|\dot{\Pi}_{\max }\right|$ between models, regardless if the mass satisfies $M_{\star} \lesssim 0.18 M_{\odot}$ or $M_{\star} \gtrsim 0.18 M_{\odot}$. Also, the $\left|\dot{\Pi}_{\max }\right|$ values are systematically larger for $g$ modes as compared with radial and $p$ modes.

In Table 13, adapted from Calcaferro et al. (2017a), we show the maximum expected rates of period change (absolute value) for dipole $g, p$, and $p-g$ mixed modes (in the case of pre-ELMVs) for low-mass WD and pre-WD models and also objects that are expected to be briefly residing in stages prior to flashes. We envisage that any future measurement of $\dot{\Pi}$ for a given pulsating low-mass pre-WD or WD star could help to establish its evolutionary status. For instance, it could be possible to distinguish a star that is in its pre-WD phase, if it is evolving in stages just prior to a $\mathrm{H} \mathrm{flash}$, or if it is

\footnotetext{
17 Hermes et al. (2013d) reported the discovery of short-period pulsations compatible with $p$ modes or radial modes in an ELMV WD (SDSS J111215.82+111745.0), but this needs to be confirmed with further observations.
} 

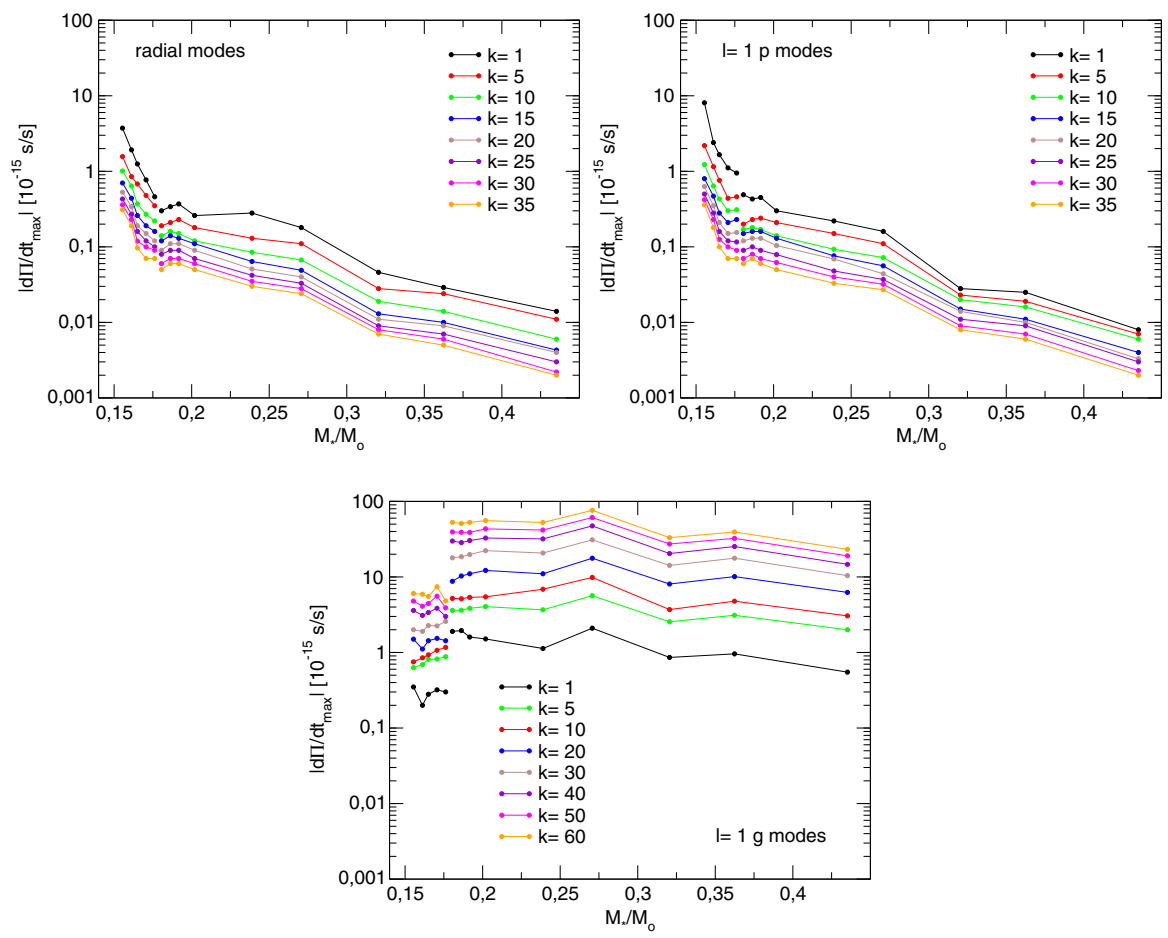

Fig. 19 Upper-left panel: absolute value of the maximum $\dot{\Pi}$ value in terms of the stellar mass corresponding to radial modes $(\ell=0)$ with selected radial orders $k$, for low-mass WD models with effective temperatures in the range $8000 \lesssim T_{\text {eff }} \lesssim 10000 \mathrm{~K}$. Upper-right panel: same as in the upper left panel, but for $\ell=1 p$ modes. Lower panel: same as in the upper left panel, but for $\ell=1 \mathrm{~g}$ modes

Table 13 Absolute value of the maximum expected rates of period change, $\left|\dot{\Pi}_{\max }\right|$ (s/s), and the sign-that indicates that the periods are decreasing or increasing with time- for nonradial $\ell=1 \mathrm{~g}$ and $p$ modes (and $p-g$ mixed modes for pre-ELMVs) corresponding to low-mass WD and pre-WD models and also objects evolving just before the CNO-cycle flashes

\begin{tabular}{lllllll}
\hline Evolutionary phase & $\left|\dot{\Pi}_{\max }\right| g$ modes & $\left|\dot{\Pi}_{\max }\right| p-g$ modes & $\left|\dot{\Pi}_{\max }\right| p$ modes \\
\hline pre-WD (pre-ELMVs) & $\sim 3 \times 10^{-13}$ & $(<0)$ & $\sim 5 \times 10^{-12}$ & $(<0)$ & $\sim 3 \times 10^{-12}$ & $(<0)$ \\
pre-CNO-cycle flashes & $\sim 3 \times 10^{-11}$ & $(<0)$ & $\ldots$ & & $\sim 2 \times 10^{-13}$ & $(>0)$ \\
WD (ELMVs) & $\sim 8 \times 10^{-14}$ & $(>0)$ & $\ldots$ & $\sim 8 \times 10^{-15}$ & $(<0)$ \\
\hline
\end{tabular}

already settled on its final cooling stage as a WD. Although less likely, it would also be possible to discriminate whether or not an ELMV star is an ELM or an LM WD. Finally, a measured value of $\dot{\Pi}$ larger than $\sim 10^{-10} \mathrm{~s} / \mathrm{s}$ would mean that the object is still evolving quickly between CNO-cycle flashes.

We close this section by briefly describing the first attempts of asteroseismology of ELMV stars. A detailed asteroseismological study of all the known and suspected ELMV stars based on the low-mass He-core WD models of Althaus et al. (2013) was presented by Calcaferro et al. (2017b). Despite ELMVs exhibiting very few periods 
and the period-to-period fits showing multiple solutions, they found that it is still possible to find asteroseismological models with $M_{\star}$ and $T_{\text {eff }}$ compatible with the values derived by spectroscopy for most cases. Due to the scarcity of periods generally exhibited by these stars, it is not feasible to assess the mean period spacing to constrain the stellar masses. A step forward in asteroseismology of ELMVs was given by Calcaferro et al. (2018b) who redid the asteroseismological analysis of these stars but this time expanding the parameter space, i.e., adopting the thickness of the $\mathrm{H}$ envelope $\left(M_{\mathrm{H}} / M_{\star}\right)$ as a free parameter, in addition to $M_{\star}$ and $T_{\text {eff }}$. They found again multiple asteroseismological solutions in all the cases, something that could be due to the few periods exhibited by these stars. Only with the inclusion of external constraints, that is, the effective temperature and surface gravity derived from spectroscopy, was it possible to adopt an asteroseismological model or a family of solutions for each star. Interestingly enough, some of the stars analyzed are better represented by asteroseismological models that harbor thin $\mathrm{H}$ envelopes. In connection with this finding, it is predicted that stable mass transfer during the binary evolution of the ELM WD progenitors leads to the formation of ELM WDs with thick H envelopes. However, the formation of ELM WDs with thinner $\mathrm{H}$ envelopes from unstable mass loss cannot be discarded. The results of Calcaferro et al. (2018b) seem to reinforce the idea that this scenario of formation could work in practice, and that the existence of ELM WDs $\left(M_{\star} \lesssim 0.18-0.20 M_{\odot}\right)$ with thin H envelopes is possible. Such WDs would not have residual $\mathrm{H}$ burning, and thus should cool very quickly. Hence, they should have had time enough to cool down to very low effective temperatures (up to $T_{\text {eff }} \sim 2500-3000 \mathrm{~K}$; see Calcaferro et al. 2018a).

Going back to the issue of asteroseismology of ELMV stars, we conclude that with the current sets of observed periods of ELMVs, it is not possible to find a unique asteroseismological solution for each star without invoking the spectroscopic determination of $T_{\text {eff }}$ and $\log g$. The situation gets worse if one or more periods are affected by large uncertainties. Since a complete set of state-of-the-art evolutionary models representative of He-core ELM WDs with different H-envelope thicknesses is employed, it is likely that a limit regarding the possibility of the asteroseismological approach to find a representative model in order to infer the internal structure of these stars has been reached. This implies the urgent necessity of detecting more pulsation modes in these stars to make more robust asteroseismological inferences. On the other hand, the discovery of additional ELMV stars is also a pressing need to better determine their internal structure and the nature of their progenitors.

\subsection{Blue large-amplitude pulsators (BLAPs)}

Recently, a new class of pulsating stars, the Blue Large-Amplitude Pulsators (BLAPs; Pietrukowicz et al. 2017), was discovered in the Optical Gravitational Lensing Experiment (OGLE; Udalski et al. 2015). The object named OGLE-BLAP-001 was the first BLAP star discovered, becoming the prototypical member of the class. It was originally misclassified as a $\delta$ Scuti variable star (OGLE-GD-DSCT-0058). At the time of writing this review, 14 BLAPs are known. BLAPs have been discovered only in the Galactic disk and bulge (high-metallicity environments), but not in the low-metallicity 


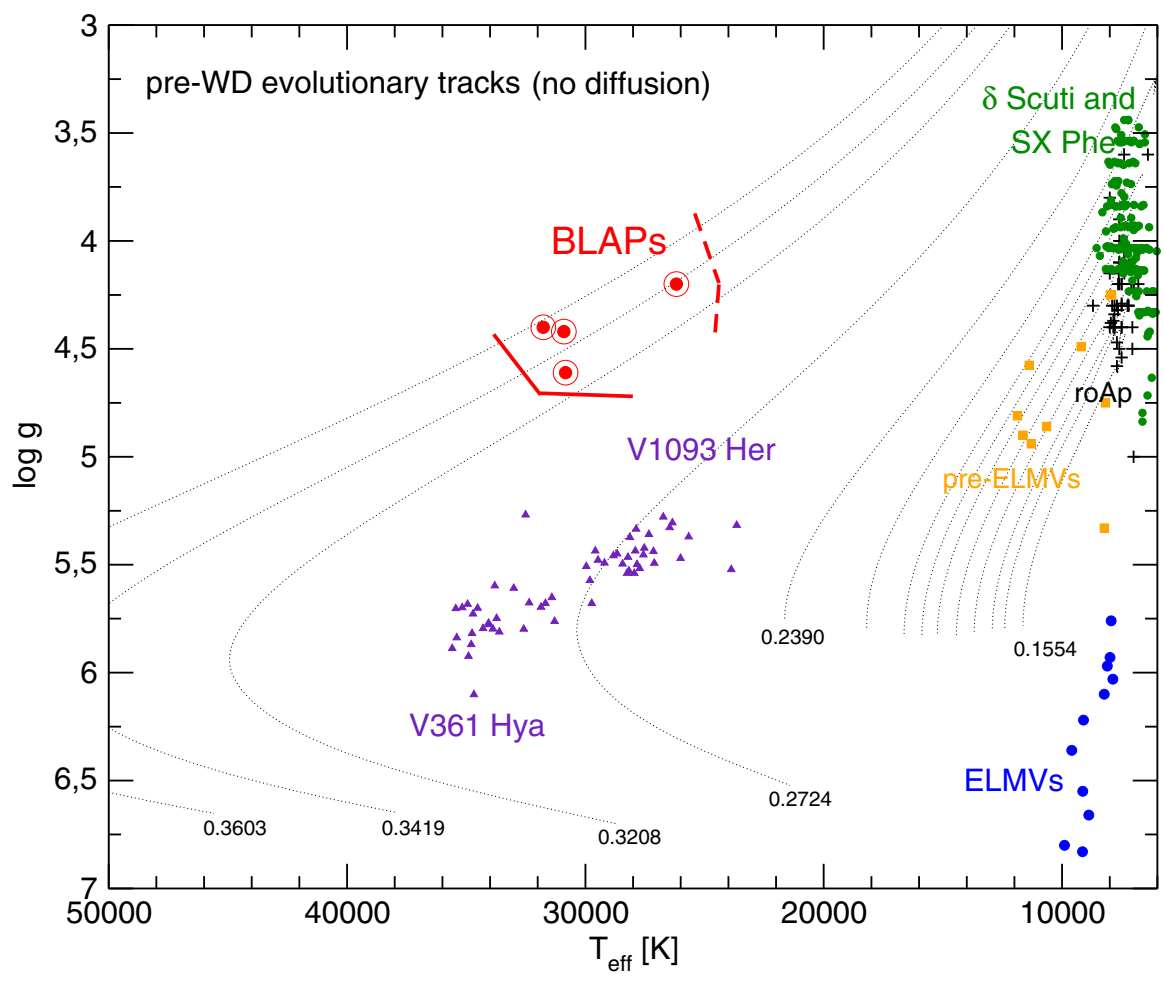

Fig. $20 T_{\text {eff }}-\log g$ diagram showing the location of four BLAP stars with measured atmospheric parameters (red dots surrounded by circles), along with other families of already known pulsating stars: ELMVs (blue dots), pre-ELMVs (orange squares), pulsating sdBs (V361 Hya and V1093 Her; violet triangles), $\delta$ Sct/SX Phe stars (green dots), and roAp stars (black plus symbols). Dotted lines correspond to low-mass He-core pre-WD evolutionary tracks for $Z=0.01$ computed neglecting element diffusion. Numbers correspond to the stellar mass of some sequences. Also included are the boundaries of the theoretical instability domain of BLAPs (thick solid and dashed red lines) of $\ell=1,2 \mathrm{~g}$ modes and radial fundamental modes $(\ell=0, k=0)$, according to Romero et al. (2018) (see the text)

environment of the Magellanic Clouds (Pietrukowicz 2018). These variables have not been observed in globular clusters nor in the Galactic halo. It seems that high metallicity is a crucial ingredient in these pulsating stars, and this is confirmed by the theoretical models. BLAPs are very hot stars, with an average effective temperature of $T_{\text {eff }} \sim 30000 \mathrm{~K}$. Their effective temperature and color change over a complete pulsation cycle, confirming that their variability is due to pulsations. Their light curves are similar to those of classical pulsators like Cepheid- and RR Lyrae-type stars that exhibit just the radial fundamental mode $(\ell=0, k=0)$, show large amplitudes $(0.2-$ $0.4 \mathrm{mag}$ ) and short periods ( $\sim 1200-2400 \mathrm{~s})$. The pulsation periods of BLAPs show a secular drift with typical values of $\dot{\Pi} / \Pi=d(\log \Pi) / d t=10^{-7}$ year $^{-1}$, both positive (increasing periods) and negative (decreasing periods). The magnitudes of the rate of period change suggest that BLAPs are stars that are evolving on nuclear timescales. Finally, BLAPs exhibit envelopes made of a mixture of $\mathrm{H}$ and He. In Fig. 20 we show the location of some BLAPs with available atmospheric parameters in the $T_{\text {eff }}-\log g$ 
diagram. For comparison, we also plot the location of other classes of known pulsating stars. Notably, BLAPs populate a region of the diagram not occupied by any category of pulsating stars previously studied. Indeed, they have effective temperatures that may be approached by the hottest $\beta$ Cep stars (not included in Fig. 20), but are also much fainter; BLAPs are much hotter than pre-ELMVs, $\delta$ Scuti/SX Phe stars, and roAp stars, although they share similar surface gravities; BLAPs have similar $T_{\text {eff }}$ as pulsating sdB stars, but are much less compact; finally, BLAPs are much hotter and less compact than ELMVs. Gaia Data Release 2 (DR2; Gaia Collaboration et al. 2018 b) has measured the parallax of 10 BLAPs, 6 of which have absolute magnitudes and intrinsic colors consistent with the temperature derived with optical spectra and theoretical predictions, whereas 4 stars have properties which appear different and may correspond to other types of pulsating variables (Ramsay 2018).

The peculiar characteristics of these pulsating stars - they show very high amplitudes that are unusual in very hot pulsating stars, they exhibit short periods and small rates of period change-pose a challenge to the theory of stellar evolution and pulsations (Pietrukowicz et al. 2017). No evolutionary/pulsational model has been proposed up to now that explains entirely the existence and the pulsational properties of BLAPs. At the outset, the evolution of single isolated low-mass stars has to be discarded to explain BLAPs, because the evolutionary timescales involved in such a scenario should be much longer than the Hubble time. Instead, Pietrukowicz et al. (2017) have proposed that binary-star evolution through stable mass transfer and/or common envelope ejection could be a plausible evolutionary channel for these intriguing pulsating stars. Pietrukowicz et al. (2017) examined two possibilities: (i) BLAPs are He-core, $\mathrm{H}$ shell burning low-mass stars $\left(\sim 0.30 M_{\odot}\right)$, or $(i i)$ BLAPs are core He-burning stars $\left(\sim 1.0 M_{\odot}\right)$.

The first detailed evolutionary and pulsational study focused on the evolutionary origin and the nature of BLAPs was that of Romero et al. (2018). They examined in detail the possibility that BLAPs are hot He-core, $\mathrm{H}$ shell burning low-mass preWD stars with masses $\sim 0.30 M_{\odot}$ coming from binary-star evolution. In Fig. 20 we display the evolutionary tracks of Althaus et al. (2013) (element diffusion not considered) corresponding to $Z=0.01$ for $M_{\star}=0.3208,0.3419$ and $0.3603 M_{\odot}$ evolving models. The location of the four BLAPs with known atmospheric parameters $\left(T_{\text {eff }}\right.$ and $g$ ) is nicely accounted for by the evolutionary tracks, suggesting that the scenario studied by Romero et al. (2018) is a plausible one. The next step taken by these authors was to try to identify the type of pulsation modes responsible for the pulsations observed in BLAPs. Specifically, they examined the possibility that periods of radial $(\ell=0)$ modes and/or nonradial $(\ell>0) p$ and $g$ modes match the periods of oscillation of BLAPs. They found that the periods of the fundamental radial mode or the first overtone $(k=0,1)$, and the periods of high radial order (25 $\lesssim k \lesssim 50$ for $\ell=1) g$ modes of solar-metallicity low-mass He-core pre-WD models $\left(M_{\star} \sim 0.32 M_{\odot}, T_{\text {eff }} \sim 31000 \mathrm{~K}, \log g \sim 4.8\right)$, are compatible with the periodicities detected in BLAPs. When tested for pulsational stability, these stellar models proved inadequate since all the pulsation modes with periods compatible with those of the BLAPs are globally stable, although substantial driving due to the $\kappa$ mechanism acting at the location of the $Z$ bump of the Rosseland opacity was found. This driving guided the authors to investigate template models 
with higher metallicity, finding that for representative models with $Z=0.05$, the radial fundamental mode and nonradial $g$ modes with the right periods are unstable, confirming the need for high metallicity (see below) as a necessary condition to explain the pulsations of BLAPs. The limits of the theoretical instability domain of BLAPs associated to $\ell=1,2 \mathrm{~g}$ modes and the radial fundamental mode corresponding to stellar models with $Z=0.05$ are displayed with thick red lines in Fig. 20.

In Fig. 21 we show the domains of instability of BLAPs in the $T_{\text {eff }}-\Pi$ diagram for radial and nonradial dipole modes corresponding to different values of the stellar mass. Similar results are found for quadrupole modes (not shown). In the case of radial modes (left panel), only the fundamental mode is unstable. The observed periodicities $(1200 \leq P \leq 2400) \mathrm{s}$ are well accounted for by the theoretical computations considering a range of stellar masses $\left(0.33 \leq M_{\star} / M_{\odot} \leq 0.36\right)$. The radial fundamental modes are the most unstable ones among the studied cases $(\ell=0,1)$. In fact, they are destabilized during very short times ( $e$-folding times) as compared with the evolutionary timescales at that stage of evolution.

The pulsation analysis of Romero et al. (2018) suggests that the pulsations of the BLAPs are better explained by the excitation of the fundamental radial mode in these stars. Indeed, the fundamental radial mode has the correct period, it is pulsationally unstable in the range of effective temperatures of interest, and it is more unstable than the nonradial $g$ modes. This conclusion is reinforced by the fact that BLAPs exhibit a single mode with large amplitude in the light curves, reminiscent of typical radial fundamental-mode pulsations. It cannot be discarded, however, that the pulsations of BLAPs correspond to nonradial $g$ modes, and that the fact that only one period is detected is due to just an insufficient observing time. On the other hand, evidence in favor of the interpretation of $g$ modes comes from the comparison of the rates of period change of BLAPs — measured by Pietrukowicz et al. (2017)—with the theoretical expectations for radial and nonradial modes. Indeed, Córsico et al. (2018) have shown that the $\dot{\Pi} / \Pi$ values of nonradial $g$ modes with high radial order $k$ are in much better agreement with the values measured in BLAPs than the fundamental radial mode. Clearly, the exact nature of the pulsation modes responsible for the variability of BLAPs remains a matter of debate.

In closing this section, we mention the issue of the puzzling super-solar metallicity required to drive pulsation modes through the $\kappa$ mechanism. In the models of Romero et al. (2018), the metallicity is globally augmented to have an enhanced $Z$ bump in the Rosseland opacity. However, it would be possible to find instability with a local enhancement of the opacity at the location of the $Z$ bump in the star. This issue has been addressed by Byrne and Jeffery (2018), who examined the pulsation instability of radial modes of single-star evolution models of post-RGB stars that have undergone a common envelope ejection in the form of a high mass-loss rate. These authors have included the effects of radiative levitation which leads to mode excitation by the $\kappa$ mechanism at $T_{\text {eff }}$ values comparable to those of BLAPs and the right period interval. By comparing with the observations, Byrne and Jeffery (2018) favor models with $\sim 0.31 M_{\odot}$ as the more likely candidates for BLAPs, in very good agreement with the scenario proposed by Romero et al. (2018). Furthermore, and more importantly, the proposal of Romero et al. (2018) that the $Z$ bump (iron and nickel) 


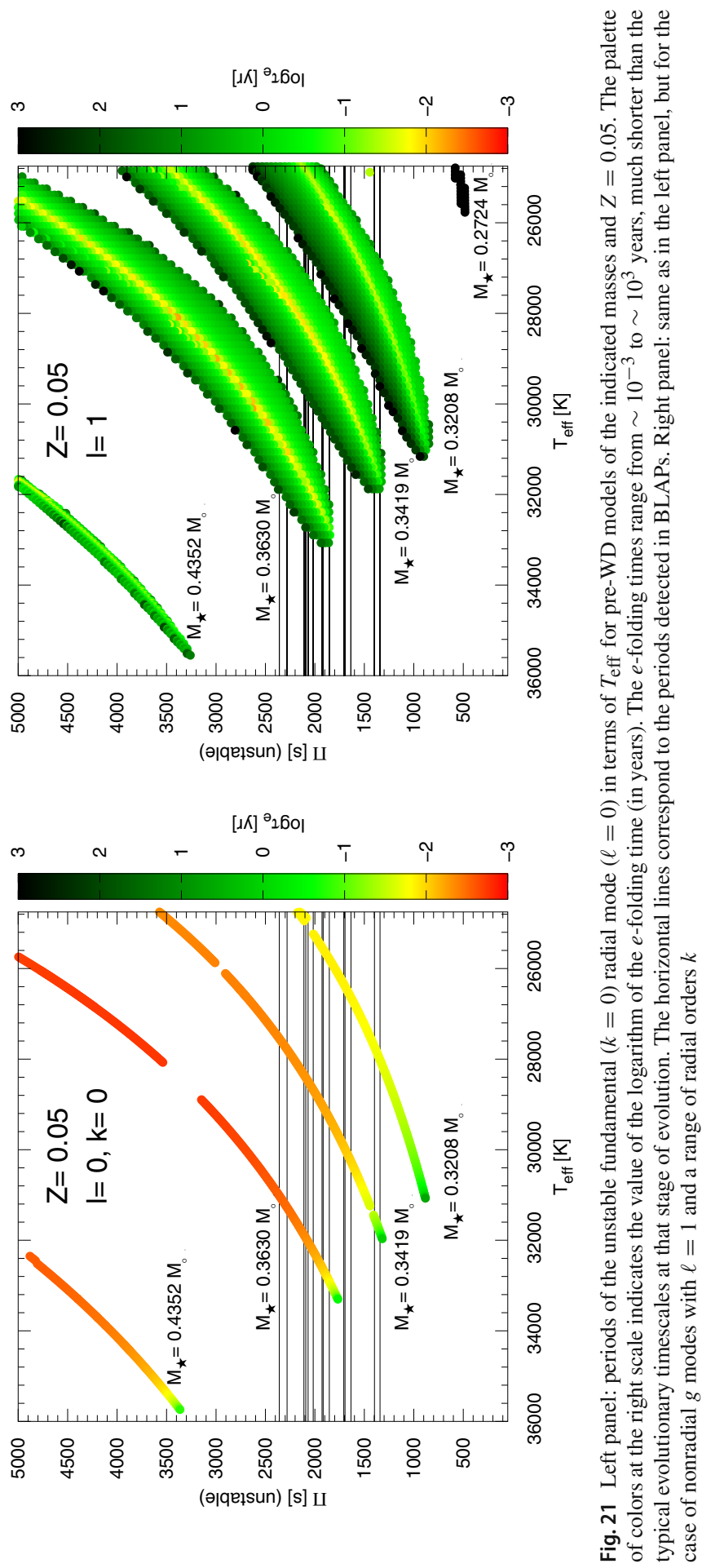


in the opacity is responsible for the excitation of pulsations in BLAPs is confirmed by the more detailed radiative-levitation calculations carried out by Byrne and Jeffery (2018).

\subsection{The sdA problem}

In the course of the analysis of the Sloan Digital Sky Survey (SDSS) Data Release 12 (DR12), thousands of objects showing H-rich spectra and sub-MS surface gravities $(4.75 \lesssim \log g \lesssim 6.5)$, but effective temperatures lower than the ZAHB ( $T_{\text {eff }} \sim$ $10000 \mathrm{~K}$ ), were identified and classified with the spectral class of subdwarf A stars ("sdAs"; Kepler et al. 2016b). The evolutionary origin of the sdAs has been elusive since their discovery. Brown et al. (2017a) suggested that sdAs could be mainly metalpoor A/F stars in the halo with an overestimated $\log g$ value, due to the use of pure $\mathrm{H}$ atmospheres to fit the spectra of these objects. However, Pelisoli et al. (2019a) showed that the addition of metals to the $\mathrm{H}$ atmosphere models does not necessarily lower the estimated $\log g$ value. At present, it is understood that sdAs consist of multiple populations, including byproducts of binary evolution (blue-stragglers, ELM WDs, and their precursors, pre-ELM WDs), metal-poor A/F dwarfs, or even stars accreted from dwarf galaxies. Preliminary constraints on sdA radii using parallaxes from Gaia DR2 suggest that at least dozens of sdAs are ELM WDs or their precursors (Pelisoli et al. 2019).

A promising avenue to study the origin and nature of sdAs is to look for pulsational variability in objects of this category. Indeed, the pulsation properties exhibited by any sdA star could help to understand their internal structure and evolutionary phase. Bell et al. (2018) carried out photometric campaigns targeting 24 sdA stars classified from SDSS spectra. They found 7 new pulsating stars, which show pulsation characteristics of ELMVs, pre-ELMVs, $\delta$ Scuti and even "RR Lyrae-like" pulsators ${ }^{18}$. Also, Pelisoli et al. (2018b) obtained time-series photometry for $21 \mathrm{sdAs}$, and found 7 new photometrically variable stars, one of which with pulsation characteristics of ELMV stars. The diversity in pulsation properties of the variable sdAs supports the idea that this population comes from a mixture of formation and evolution scenarios. Also, it seems that the fraction of variable stars among the sdAs is large, something that could be exploited by asteroseismology to shed some light on their internal structure and evolutionary status.

\subsection{WD pulsators as cosmic laboratories for fundamental physics}

WD asteroseismology constitutes a novel tool for applications of WDs to fields beyond stellar astrophysics. A vivid example of this is the application of pulsating WDs to constrain properties of elementary particles and to test the possible variation of fundamental constants. In this Section, we summarize the use of pulsating WDs to assess stringent constraints on the mass of the axions, the magnetic dipole moment of the neutrino, and the secular rate of variation of the gravitational constant, $G$. For a back-

$\overline{18}$ They have been called "binary evolution pulsators" by Smolec et al. (2013). 
ground about the application of stars to the study of weakly interacting particles and the possible drift of fundamental constants, we recommend the reader to consult the excellent review articles by Raffelt (1990) and García-Berro et al. (2007), respectively. The application of pulsating WDs to study theories with large extra dimensions, WIMPs (weakly interacting massive particles), axions and the possible time variation of $G$ has been discussed by Malec and Biesiada (2013) (see, also, Biesiada and Malec 2002, 2003).

\subsubsection{Upper bounds on the axions mass}

Axions are hypothetical pseudo Nambu-Goldstone bosons (that is, bosons with a tiny mass; Raffelt 1996) that were postulated by Peccei and Quinn (1977), Weinberg (1978), and Wilczek (1978) to solve the long-standing problem in particle physics known as the "strong CP problem" (Kim and Carosi 2010). The strong CP problem consists of non-violation of the charge-parity (CP) symmetry ${ }^{19}$ in strong interactions. The breaking of this symmetry is predicted by quantum chromodynamics (QCD) ${ }^{20}$. At present, axions are the focus of a plethora of theoretical and experimental investigations aimed at proving their existence (Irastorza and Redondo 2018). Despite much effort, this elusive particle has not yet been detected. Giannotti (2017), Gianninas et al. (2017), and Hoof et al. (2019) present updated accounts of observational hints from astrophysics pointing to the existence of stellar energy losses beyond the ones accounted for by neutrino emission, and future experiments aimed at detecting axions. A property of utmost importance of axions is the axion mass $\left(m_{\mathrm{a}}\right)$. The importance of knowing the mass of the axion lies in the fact that, depending on its value, they could contribute substantially or not to the cold dark matter of the Universe. Axions are electrically neutral, and they interact very weakly with normal matter and radiation. There exist several axion models, the most important one in the WD context being the DFSZ model (Dine-Fischler-Srednicki-Zhitnitsky; Zhitnitsky 1980; Dine et al. 1981), where axions couple to charged leptons like electrons with a strength defined by the dimensionless coupling constant, $g_{\mathrm{ae}}$, being $g_{\mathrm{ae}}=2.8 \times 10^{-14} \mathrm{~m}_{\mathrm{a}} \cos ^{2} \beta$, where $\cos \beta$ is undetermined.

Since the axion mass is not predicted by the theory that postulates its existence (Raffelt 2007), it must be derived from either terrestrial experiments (Redondo 2016), or indirectly using well-studied properties of stars (Vysotsky et al. 1978; Raffelt 1996). Here, we describe how pulsating WDs can be used to constrain $m_{\mathrm{a}}$. The degenerate cores of WDs contain plenty of free electrons (Althaus et al. 2010b); therefore, axions would be abundantly produced in their interiors (Raffelt 1986). In stars, the energy drain by weakly interacting particles (such as axions) is equivalent to a local energy sink. Indeed, the stellar energy generation rate can be written $\varepsilon_{\text {eff }}=\varepsilon_{\text {nuc }}-\varepsilon_{v}-\varepsilon_{\mathrm{a}}$, where $\varepsilon_{\text {nuc }}$ is the nuclear burning rate, $\varepsilon_{v}$ is the neutrino loss, and $\varepsilon_{\mathrm{a}}$ is the axion loss. Since WDs are strongly degenerate and generally do not have relevant nuclear

\footnotetext{
19 The CP symmetry establishes that the laws of physics should be the same if particles were replaced with their antiparticles (C symmetry) and their spatial coordinates were inverted (P symmetry) (Luders 1954; Pauli et al. 1995).

20 The name of axion comes from the Axion laundry detergent, and was introduced by Frank Wilczek "to clean QCD from the CP problem".
} 
energy sources $\left(\varepsilon_{\text {nuc }}=0\right)^{21}$, their evolution consists of a slow cooling process in which the gravothermal energy release is the main energy source regulating their evolution (Mestel 1952). Thus, in WDs the emission of axions means speeding up the cooling, with observable consequences. The axion emission rate in WDs is $\varepsilon_{\mathrm{a}} \propto g_{\mathrm{ae}}^{2}$, with Bremsstrahlung processes being the dominant mechanism. Therefore, the more massive the axions are, the larger the axion emission is. Since axions can freely escape from the interior of WDs, their emission would accelerate cooling, with more massive axions producing larger cooling rates. In the case of pulsating WDs, the cooling of the star is reflected by a secular change of the pulsation periods. The rate of period change $(\dot{\Pi} / \Pi)$ is connected to the rate of change of the core temperature $(\dot{T} / T)$ and the rate of variation of the stellar radius $\left(\dot{R}_{\star} / R_{\star}\right)$ through the order-of-magnitude relation: $\dot{\Pi} / \Pi \approx-(\dot{T} / T)+\left(\dot{R}_{\star} / R_{\star}\right)$ (Winget et al. 1983). For ZZ Ceti and V777 Her stars, $\dot{T}<0$ and $\dot{R}_{\star} \approx 0$, so that $\dot{\Pi}>0$ and the periods should lengthen with cooling, something that is confirmed by observations.

If a pulsating WD emits axions, then it should cool faster than otherwise expected, with the acceleration of cooling being proportional to the mass of the axion. The enhancement of cooling of the WD should be reflected in a larger rate of period change. This is the principle by which it is possible to infer constraints on the mass of the axion through pulsating WDs. This approach was first employed by Isern et al. (1992) considering the archetype ZZ Ceti star G117-B15A. They assessed the rate of period change of the largest amplitude mode $(\Pi \sim 215 \mathrm{~s})$ of this star by means of a semi-analytic treatment considering the evolution of DA WDs with and without axion emission, and compared the theoretical values of $\dot{\Pi}$ for increasing values of $m_{\mathrm{a}}$ to the observed rate of change of period with time of G117-B15A. They found that to match the observed $\dot{\Pi}$, the axion mass should be $m_{\mathrm{a}} \cos ^{2} \beta \leq 8.7 \mathrm{meV}$ ( $g_{\mathrm{ae}} \leq$ $\left.2.4 \times 10^{-13}\right)$. Later, the approach was refined by Córsico et al. (2001) and BischoffKim et al. (2008b) considering detailed asteroseismological analyses of G117-B15A, and obtaining $m_{\mathrm{a}} \cos ^{2} \beta \leq 4.4 \mathrm{meV}\left(g_{\mathrm{ae}} \leq 1.2 \times 10^{-13}\right)$ and $m_{\mathrm{a}} \cos ^{2} \beta \leq 26.5 \mathrm{meV}$ $\left(g_{\mathrm{ae}} \leq 7.4 \times 10^{-13}\right)$, respectively. The asteroseismological models for G117-B15A employed in those works, however, were not robust enough, and the observed values of the rate of period change had large uncertainties. The situation strongly improved with a new measurement of the observed value of $\dot{\Pi}$ for this star $\left((4.19 \pm 0.73) \times 10^{-15} \mathrm{~s} / \mathrm{s}\right.$; Kepler 2012), and a new asteroseismological model for G117-B15A (Romero et al. 2012). Armed with these new determinations, Córsico et al. (2012b) obtained a new upper bound of the axion mass. In Fig. 22 we show the rate of period change for the mode with period of $\sim 215 \mathrm{~s}(\ell=1, k=2)$ of the asteroseismological model for G117-B15A derived by Romero et al. (2012) in terms of the axion mass, where the errors in $\dot{\Pi}$ due to only internal uncertainties in the asteroseismological procedure are included. The true uncertainties of the theoretical $\dot{\Pi}$ could be larger if the uncertainties in the WD previous evolution were taken into account (see De Gerónimo et al. 2017, 2018). The observed value with its uncertainties is also plotted. The comparison of the theoretical rate of period change associated with the asteroseismological model to the observed one suggests the existence of an additional cooling mechanism in

21 Exceptions to this assertion are WDs coming from low-metallicity progenitors (Miller Bertolami et al. 2013; Althaus et al. 2015) and ELM WDs (Althaus et al. 2013). 


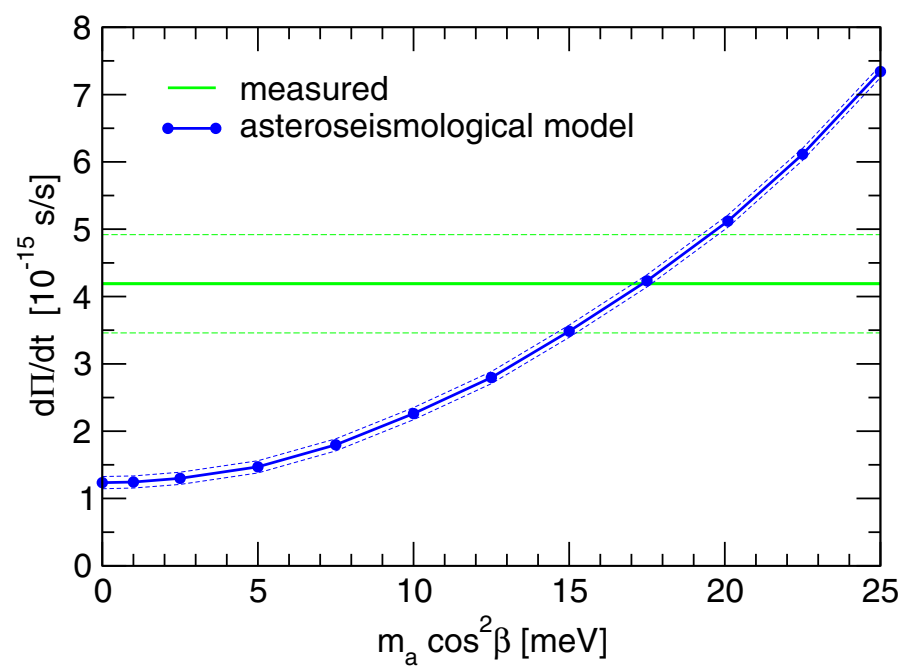

Fig. 22 Rate of period change for the mode with $\ell=1, k=2$ of the asteroseismological model of G117-B15A (solid blue curve with dots) in terms of the axion mass. Dashed curves represent the errors in $\dot{\Pi}$ due to internal uncertainties in the asteroseismological procedure. The horizontal green lines indicate the observed value with its corresponding uncertainties

this pulsating WD, consistent with axions of mass of $m_{\mathrm{a}} \cos ^{2} \beta \leq 17.4 \mathrm{meV}\left(g_{\mathrm{ae}} \leq\right.$ $\left.4.9 \times 10^{-13}\right)$. This is similar to the constraint obtained from RGB stars in globular clusters $\left(g_{\mathrm{ae}} \leq 4.3 \times 10^{-13}\right.$, Viaux et al. 2013a).

Similar analyses have been carried out considering other ZZ Ceti stars for which the rate of period change has been measured. Specifically, Córsico et al. (2012c) obtained an upper limit of $m_{\mathrm{a}} \cos ^{2} \beta \leq 17.1 \mathrm{meV}\left(g_{\mathrm{ae}} \leq 4.8 \times 10^{-13}\right)$ considering the prototype ZZ Ceti star R548 from the rate of period change for the mode with period $\sim 212 \mathrm{~s}$ measured by Mukadam et al. $(2013), \dot{\Pi}=(3.3 \pm 1.1) \times 10^{-15} \mathrm{~s} / \mathrm{s}$. A third ZZ Ceti star, L19-2, was employed to infer an upper bound for the axion mass. Indeed, Córsico et al. (2016b) derived $m_{\mathrm{a}} \cos ^{2} \beta \leq 25 \mathrm{meV}\left(g_{\mathrm{ae}} \leq 7 \times 10^{-13}\right)$ from the value of the rate of period change of the modes with periods $113 \mathrm{~s}$ and $192 \mathrm{~s}$ of $\dot{\Pi}=(3.0 \pm 0.6) \times 10^{-15} \mathrm{~s} / \mathrm{s}$ measured by Sullivan and Chote (2015). A fourth pulsating WD, the V777 Her star PG 1351+489, has been scrutinized for a rate of period change. Redaelli et al. (2011) have measured a preliminary value of the rate of period change of $\dot{\Pi}=(2.0 \pm 0.9) \times 10^{-13} \mathrm{~s} / \mathrm{s}$ for the period at $\sim 490 \mathrm{~s}$ of this star, and this value has has been employed by Battich et al. (2016) to constrain the axion mass: $m_{\mathrm{a}} \cos ^{2} \beta \leq 19.5 \mathrm{meV}\left(g_{\mathrm{ae}} \leq 5.5 \times 10^{-13}\right)$. An alternative way to present the results of the axion analyses from WD pulsations is in terms of $\alpha_{26}=\left(g_{\mathrm{ae}} / 10^{-13}\right)^{2} / 4 \pi$, rather than in terms of $g_{\mathrm{ae}}$ or $m_{\mathrm{a}}$. In fact, the emission rate is proportional to $g_{\mathrm{ae}}^{2}$. In Table 14 (M. Giannotti, private communication) we depict the $1 \sigma$ and $2 \sigma$ intervals of $\alpha_{26}$ for G117-B15A (Romero et al. 2012), R548 (Córsico et al. 2012c), L19-2 (Córsico et al. 2016b), and PG1351+489 (Battich et al. 2016).

Another method to determine the mass of the axion employs the WD luminosity function (WDLF), which is defined as the number of WDs per unit bolometric magni- 
Table $141 \sigma$ and $2 \sigma$ intervals in $\alpha_{26}$ as inferred from the WD

\begin{tabular}{lllll}
\hline Star & $\Pi(\mathrm{s})$ & $\alpha_{26}$ & $1 \sigma$ & $2 \sigma$ \\
\hline G117-B15A & 215 & 1.89 & 0.48 & 0.95 \\
R548 & 212 & 1.84 & 0.93 & 1.85 \\
L19-2 & 113 & 2.08 & 1.35 & 2.70 \\
L19-2 & 192 & 0.50 & 1.21 & 2.43 \\
PG1351+489 & 490 & 0.36 & 0.38 & 0.76 \\
\hline
\end{tabular}
pulsation analyses

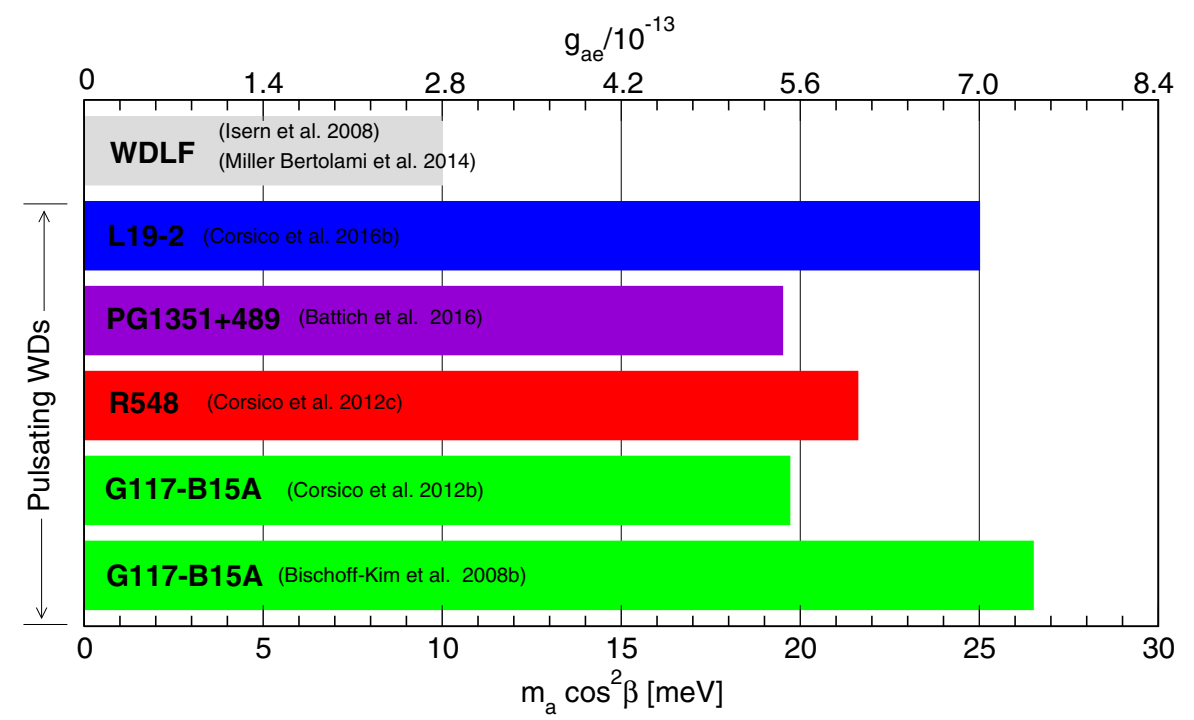

Fig. 23 Upper limits for the axion mass from pulsating WDs and from the WDLF

tude and unit volume. The shape of the WDLF is sensitive to the characteristics of the cooling of WDs. This was exploited by Isern et al. (2008) who found that when axion emission is included in the WD models, the agreement between the theoretical and the observed WDLFs significantly improves (Isern et al. 2008, 2009). More recent work employs new WDLFs of the Galactic disk and state-of-the-art theoretical WD cooling sequences (Miller Bertolami et al. 2014). A tight constraint for the axion mass has been obtained by Hansen et al. (2015) from the WDLF of the globular cluster 47 Tucanae, $m_{\mathrm{a}} \leq 4.1 \mathrm{meV}\left(g_{\mathrm{ae}} \sim 8.4 \times 10^{-14}\right)$ to a $95 \%$ confidence limit. On the other hand, Isern et al. (2018) have computed the theoretical WDLF of the thin and thick disk, and of the stellar halo including axion emission, and compared them with the existing observed WDLFs. All these studies do not discard the existence of extracooling in WDs, compatible with axions with an upper limit for the mass of $\sim 10 \mathrm{meV}$ $\left(g_{\mathrm{ae}} \sim 2.4 \times 10^{-13}\right)$. We summarize the most recent DFSZ axion-mass limits derived from pulsating WDs as well as employing the WDLF in Fig. 23. The WDLF approach seems to discard axion masses larger than $\sim 10 \mathrm{meV}$ (in agreement with the SN1987A determination); so, this method seems to be more stringent than that using pulsating WDs (axion masses $\lesssim 25 \mathrm{meV}$ ). However, there exist important uncertainties in the 
determination of the asteroseismological models, connected with the prior evolution of WDs and the microphysics (EoS, opacities), and also large uncertainties in the theoretical and observed WDLFs. Therefore, taking into account the uncertainties affecting both approaches, the results obtained from the WDLF and asteroseismological models of pulsating WDs are compatible. We conclude from both independent methods that the existence of extra-cooling in WDs, compatible with the emission of axions with masses lower than $\sim 10-25 \mathrm{meV}$ cannot be discarded. Both methods are expected to become more precise with more pulsating WDs having $\dot{\Pi}$ measured (for instance, through the TESS mission), and also substantial improvements in the determining of the observed WDLF from the Gaia collaboration.

\subsubsection{Constraints on the neutrino magnetic dipole moment}

As often happens in physics, the conservation of a given fundamental quantity requires the existence of a particle hitherto unknown. Neutrinos have not been the exception. The existence of neutrinos was first postulated by W. Pauli in 1930 to explain the conservation of energy, momentum, and angular momentum in $\beta$-decays (Raffelt 1996). In the frame of the Standard Model (SM) of particle physics, neutrinos are massless, electrically neutral, have zero decay rate, and, in particular, they have zero dipole moment. This simple characterization of neutrinos fails when the observed neutrino mixing and oscillation have to be explained. In this case, it is necessary to go beyond the SM, allowing a finite neutrino mass, neutrino decays, and in particular, a non-zero magnetic dipole moment, $\mu_{v}$ (Raffelt 1996). The neutrino magnetic dipole moment was computed for the first time by Fujikawa and Shrock (1980).

Neutrino emission represents an efficient energy-loss mechanism in a variety of stars, from low-mass red giants and horizontal-branch stars to WDs, neutron stars and core-collapse supernovae. In the case of WDs - and other objects at advanced stages of stellar evolution like the cores of red giant stars-neutrinos are produced through thermal effects, without nuclear reactions being involved ${ }^{22}$. In particular, in the case of pre-WDs and very hot WDs, neutrinos are produced mainly as a result of plasmon decay processes $[\gamma \rightarrow \bar{v} v]$, in which a neutrino $(\nu)$ and anti-neutrino $(\bar{v})$ pair is generated due to an indirect coupling between neutrinos and photons $(\gamma)$ through electrons in a plasma. If neutrinos have a non-zero magnetic dipole moment, then a direct coupling between neutrinos and the photons (electromagnetic field) is allowed. In this case, plasmon emission processes have to be much more efficient.

The fact that plasma neutrino emission is the dominant cooling mechanism in preWDs and hot WDs (Raffelt 1996; Winget et al. 2004) implies that the evolutionary timescale of these stars is sensitive to this mechanism. In other words, the larger the neutrino plasma emission, the faster the evolution of these stars. This fact offers the possibility of employing the observed properties of pre-WD and hot WD stars to constrain the possible amount of anomalous energy loss, if it exists, and then the magnitude of the neutrino magnetic dipole moment (Bernstein et al. 1963; Raffelt 2012).

\footnotetext{
22 This is at variance with solar neutrinos, which are the result of nuclear fusion. For the Sun, thermal neutrino emission is negligible (Raffelt 1996).
} 


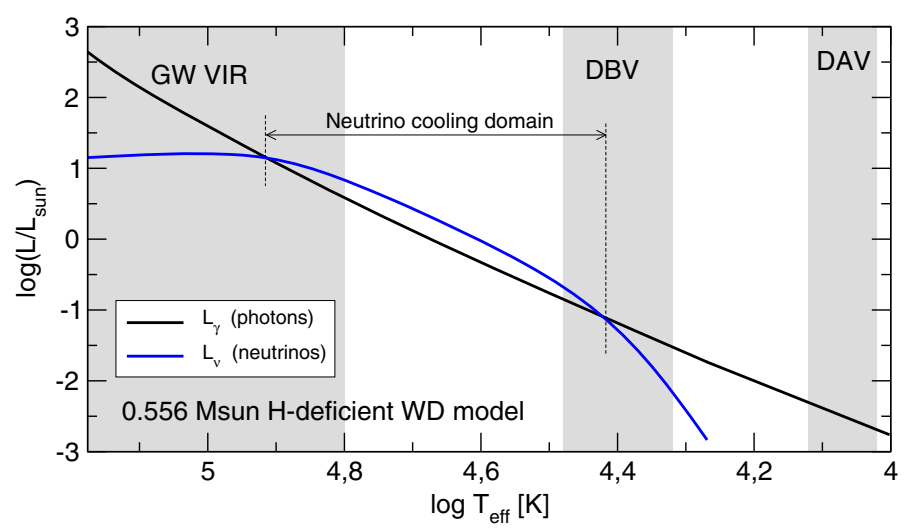

Fig. 24 Neutrino (blue line) and photon (black line) luminosities for a H-deficient WD model with $M_{\star}=$ $0.556 M_{\odot}$. The shaded areas emphasize the location of the instability domains of GW Vir, V777 Her (DBV) and ZZ Ceti (DAV) stars

A way to constrain the neutrino magnetic dipole moment from pre-WDs and hot WDs is provided by asteroseismology. In particular, the DBV (V777 Her) instability strip $\left(22400 \mathrm{~K} \lesssim T_{\text {eff }} \lesssim 32000 \mathrm{~K}\right)$ and the $\mathrm{GW}$ Vir instability domain $(80000 \mathrm{~K} \lesssim$ $T_{\text {eff }} \lesssim 180000 \mathrm{~K}$ ) partly overlap with the $T_{\text {eff }}$ interval in which plasmon neutrino emission controls the evolution of H-deficient WDs. This is borne out in Fig. 24, where we plot the neutrino and photon luminosities of a $0.556 M_{\odot} \mathrm{H}$-deficient WD model in terms of the effective temperature. From $\log T_{\text {eff }} \sim 4.9$ down to $\log T_{\text {eff }} \sim 4.4$, neutrino luminosity exceeds photon luminosity. The evolutionary timescale can be derived, in principle, by measuring the rates of period changes. Therefore, in the overlapping regions, the magnitude of the rates of period change of GW Vir and V777 Her stars is expected to be influenced by plasmon neutrino emission. This means that, if a non-vanishing neutrino magnetic dipole moment exists, the value of $\dot{\Pi}$ should be enhanced as compared with the case in which $\mu_{v}=0$, and this excess in the period drift provides a constraint to $\mu_{\nu}$. This approach is exactly the same as that adopted to derive upper bounds to the axion mass (see Sect. 3.9.1).

In the GW Vir regime, the prospect of using a measured $\dot{\Pi}$ to constrain plasmon neutrino emission was explored by O'Brien and Kawaler (2000), and in the V777 Her (DBV) domain by Winget et al. (2004). In the case of GW Vir stars, the pulsating star PG 0122+200 could, in principle, be an appropriate candidate to place constraints on the plasmon neutrino emission rate on the basis of an observed value of $\dot{\Pi}$. This is because, at the effective temperature of this pulsating $\operatorname{star}\left(T_{\text {eff }}=80000 \pm 4000 \mathrm{~K}\right)$, the neutrino luminosity should be comparable to the photon luminosity. However, Vauclair et al. (2011) found $\dot{\Pi}$ values for the periods of this star that are 100-1000 times larger than the value expected from the asteroseismological model derived by Córsico et al. (2007b). This period drift cannot be explained by neutrino cooling only, so that probably, another mechanism is at work. Vauclair et al. (2011) suggest that the resonant mode coupling induced within triplets by rotation could be such a mechanism. In short, PG 0122+200 is not useful for constraining neutrino emission. In the case of V777 Her stars, the pulsating star EC 20058-5234 ( $T_{\text {eff }}=25000-27000 \mathrm{~K}$; Chote et al. 2013) 


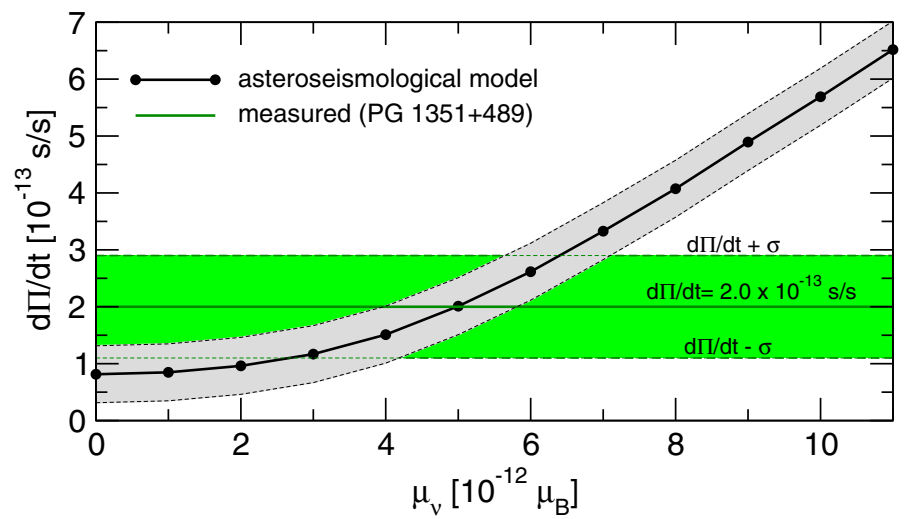

Fig. 25 Theoretical $\dot{\Pi}$ value corresponding to the period of $489 \mathrm{~s}$ of the DBV star PG $1351+489$ in terms of the neutrino magnetic dipole moment (black solid curve and dots), and the measured value (horizontal green solid line) with its corresponding $1 \sigma$ uncertainties $\left( \pm 0.9 \times 10^{-13} \mathrm{~s} / \mathrm{s}\right.$, dashed lines $)$

could be a good candidate for exploring the efficiency of plasmon neutrino emission. From theoretical grounds, neutrino luminosity should be a factor of $\sim 3$ larger than the photon luminosity in this star. Unfortunately, no measurement of $\dot{\Pi}$ is possible because the long-term period stability is not good enough (Sullivan 2017). Another V777 Her star that could place constraints on neutrino emission is PG 0112+104 (Hermes et al. 2017b), the hottest DBV of the class $\left(T_{\text {eff }}>30000 \mathrm{~K}\right)$, although a measurement of the rate of change of its periods is not available yet. Fortunately, it has been possible to determine a preliminary value of $\dot{\Pi} \sim(2.0 \pm 0.9) \times 10^{-13} \mathrm{~s} / \mathrm{s}$ for the largest amplitude mode $(\Pi \sim 489 \mathrm{~s})$ of another V777 Her star, the pulsating star PG 1351+489 (Redaelli et al. 2011). Regrettably, with a $T_{\text {eff }} \sim 22000-26000 \mathrm{~K}$, this star could be too cool to allow measurement of the normal plasmon emission rate, but it can be still employed for detecting anomalous neutrino emission.

Córsico et al. (2014) employed the estimate of the rate of period change for PG $1351+489$ to derive an upper limit to the neutrino magnetic dipole moment. Specifically, these authors computed the anomalous energy loss due to the existence of a magnetic dipole moment, $\varepsilon_{v}^{\mathrm{mdm}}$, from the plasmon neutrino emission, $\varepsilon_{\nu}^{\mathrm{p}}$, employing the scaling relation of Haft et al. (1994): $\varepsilon_{v}^{\mathrm{mdm}}=0.318 \mu_{12}^{2}\left(10 \mathrm{keV} / \hbar \omega_{\mathrm{p}}\right)^{2}\left(Q_{2} / Q_{3}\right) \varepsilon_{v}^{\mathrm{p}}$, where $\mu_{12}=\mu_{v} / 10^{12} \mu_{\mathrm{B}}$ ( $\mu_{\mathrm{B}}$ is the Bohr magneton). Córsico et al. (2014) compared the theoretical $\dot{\Pi}$ value for asteroseismological models considering increasing values of $\mu_{v}$ with the observed rate of period change of PG 1351+489, and assessed the possible existence of additional cooling by neutrinos with magnetic dipole moment. Fig. 25 displays the rate of period change of the period $\Pi=489.3 \mathrm{~s}$ (for $\mu_{v}=0$ ), in terms of increasing values of $\mu_{v}$, corresponding to the asteroseismological model for PG $1351+489$. The estimate of the rate of period change of the 489 s period of PG $1351+489$ and its uncertainties are also shown. These results suggest the existence of additional cooling in this V777 Her star, consistent with a non-zero magnetic dipole moment with an upper limit of $\mu_{v} \lesssim 7 \times 10^{-12} \mu_{\mathrm{B}}$. A similar analysis, but without considering an asteroseismological model for PG $1351+489$, but instead the spectroscopically derived effective temperature of this star, indicates an upper limit of $\mu_{v} \lesssim$ 
$9 \times 10^{-12} \mu_{\mathrm{B}}$. In summary, a conservative limit of $\mu_{v} \lesssim 10^{-11} \mu_{\mathrm{B}}$ can be adopted as the main result of the analysis. This constraint is a bit less restrictive than the upper bound derived from the Galactic WDLF, of $\mu_{v} \lesssim 5 \times 10^{-12} \mu_{\mathrm{B}}$ (Miller Bertolami 2014), the upper bound for $\mu_{v}$ derived from the analysis of red giants from the color-magnitude diagram of the Galactic globular cluster M5, $\mu_{v} \lesssim 4.5 \times 10^{-12} \mu_{\mathrm{B}}$ corresponding to the $2 \sigma$ limit (Viaux et al. 2013a, b), and the $95 \%$ confidence limit of $\mu_{v} \lesssim 3.4 \times 10^{-12} \mu_{\mathrm{B}}$ derived by Hansen et al. (2015) from the WDLF of the globular cluster 47 Tucanae.

\subsubsection{Limits on the secular rate of change of the gravitational constant}

According to the General Relativity theory, the gravitational constant, $G$, does not vary with time or location. However, some alternative theories of gravity predict that the gravitational constant is both time and space dependent (Uzan 2003; García-Berro et al. 2007). If these theories are correct, it is expected that the gravitational constant would vary slowly over long timescales. A large variety of methods aimed to place limits on a possible variation of $G$ exists. The most restrictive upper bounds are derived using Lunar Laser Ranging $\left(\dot{G} / G=(0.2 \pm 0.7) \times 10^{-12}\right.$ year $^{-1}$, Hofmann et al. 2010) and Big Bang nucleosynthesis $\left(-0.3 \times 10^{-12} \lesssim \dot{G} / G \lesssim 0.4 \times 10^{-12}\right.$ year $^{-1}$, Copi et al. 2004). Other constraints, somewhat less restrictive, come from the WDLF in clusters $\left(\dot{G} / G \lesssim 1.8 \times 10^{-12}\right.$ year $^{-1}$, García-Berro et al. 2011) and also from the Hubble diagram of Type Ia supernovae $\left(\dot{G} / G \lesssim 1 \times 10^{-11}\right.$ year $^{-1}$ at $z \sim 0.5$, Garcia-Berro et al. 2006), see also Mould and Uddin $(2014)\left(-3 \times 10^{-11} \lesssim \dot{G} / G \lessgtr 7.3 \times 10^{-11}\right.$ year $\left.^{-1}\right)$.

WDs are ideal targets to detect a hypothetical secular change of $G$ because they have very long evolutionary timescales, so that the cumulative effect of a slightly changing $G$ should lead sizable imprints in their evolutionary properties. This has been investigated by Althaus et al. (2011), who showed that the mechanical structure and the energy balance of WDs are strongly modified by a varying the gravitational constant, and the impact is more pronounced for more massive WDs. Córsico et al. (2013) have taken advantage of the sensitivity of the evolutionary timescale of WDs to $G$ to put limits on its rate of variation by comparing the theoretical $\dot{\Pi}$ obtained taking into account the effects of a running $G$ with the measured rates of period change of the ZZ Ceti stars G117-B15A and R548. The rates of period change measured for the $\sim 215 \mathrm{~s}$ mode $(\mathrm{G} 117-\mathrm{B} 15 \mathrm{~A})$ and the $\sim 212 \mathrm{~s}$ mode (R548) are larger than those predicted by the asteroseismological models for these stars, derived by assuming $\dot{G}=0$. Hypothesizing that this discrepancy can be attributed exclusively to a variable $G$, assuming for simplicity that $\dot{G} / G$ is constant, considering for simplicity that $\dot{G}<0$, and adopting different values for this quantity, these authors derived upper limits for the rate of variation of the gravitational constant of $\dot{G} / G=-1.8 \times$

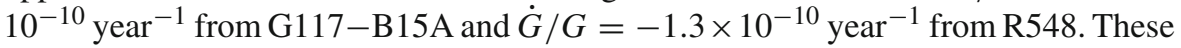
values are completely compatible with each other, and are currently less restrictive than those derived using other methods such as Lunar Laser Ranging and Big Bang nucleosynthesis. They are comparable to the upper limits derived from Hubble diagram of Type Ia supernovae and the WDLF. These bounds could be improved if the $\dot{\Pi}$ value were measured for a massive pulsating WD, since the effects of a varying $G$ are stronger for the more massive WDs (Althaus et al. 2011). 


\section{Summary}

The study of WD stars exceeds by far the scope of the theory of stellar evolution. Examples abound of WD applications to various areas of astrophysics, such as cosmochronology, and also as laboratories to study exotic physics. Fortunately, WDs go through certain stages during which the pulsations they experience allow to "see" their interiors, thus enabling to elucidate their inner structure and evolutionary stage through asteroseismology. The progress in the study of pulsating WDs has been remarkable in the last decade. This progress has been partly boosted with both the advent of observations from space such as the Kepler and K2 missions, and the generation of new detailed evolutionary models, along with the development of powerful asteroseismological techniques. Obviously, the refinement of the observations has led to new challenges for the theoreticians, being perhaps the best example the exciting detection of outbursts in cool ZZ Ceti stars.

With the availability of parallax measurements from the Gaia collaboration, and with the arrival of new data from the TESS mission, the field of WD asteroseismology will surely experience an even greater revolution in a few years. These circumstances force the members of the WD community to be ready to interpret new and unexpected data, which will ultimately lead to correcting the current theoretical models. Fields such as the dating of stellar populations through cosmochronology, the theory of accretion in WDs, the physics of matter under extreme conditions (such as crystallization), as well as the study of fundamental particles (both hypothetical and real), will ultimately be strongly benefited.

Acknowledgements We thank our referees (Pier-Emmanuel Tremblay and an anonymous referee), for their very relevant comments and suggestions that largely improved the content of the paper. We warmly thank Tiara Battich, Leila M. Calcaferro, and Francisco C. De Gerónimo, members of the La Plata Stellar Evolution and Pulsation Research Group, (http://evolgroup.fcaglp.unlp.edu.ar/) for their valuable suggestions concerning the presentation of the material of this review. We also thank Keaton J. Bell, Paul A. Bradley, Márcio Catelan, Stéphane Charpinet, Maurizio Giannotti, Steve D. Kawaler, and Don E. Winget for the invaluable effort of reading the manuscript critically and suggesting changes that profoundly enriched the content of this article. We particularly thank Keaton J. Bell for being so kind in preparing Figure 12 for this review. Special thanks also to Paul A. Bradley, Márcio Catelan, and Leila M. Calcaferro for editing the text and greatly improving the presentation. Finally, we warmly appreciate the analysis kindly provided by Maurizio Giannotti on axion constraints. This paper is dedicated to the memory of Enrique GarcíaBerro, our great friend and mentor. Part of this work was supported by AGENCIA through the Programa de Modernización Tecnológica BID 1728/OC-AR, and by the PIP 112-200801-00940 grant from CONICET. M3B is partially supported by ANPCyT through grant PICT 2016-0053, and by the MinCyT-DAAD bilateral cooperation program through grant DA/16/07. This research has made intensive use of NASA's Astrophysics Data System.

\section{References}

Aerts C, Christensen-Dalsgaard J, Kurtz DW (2010) Asteroseismology. Astronomy and Astrophysics Library, Springer, Dordrecht, https://doi.org/10.1007/978-1-4020-5803-5

Althaus LG, Miller Bertolami MM, Córsico AH, García-Berro E, Gil-Pons P (2005) The formation of DA white dwarfs with thin hydrogen envelopes. Astron Astrophys 440:L1-L4. https://doi.org/10.1051/ 0004-6361:200500159. arXiv:astro-ph/0507415 
Althaus LG, García-Berro E, Isern J, Córsico AH, Rohrmann RD (2007) The age and colors of massive white dwarf stars. Astron Astrophys 465:249-255. https://doi.org/10.1051/0004-6361:20066059. arXiv:astro-ph/0702024

Althaus LG, Córsico AH, Bischoff-Kim A, Romero AD, Renedo I, García-Berro E, Miller Bertolami MM (2010a) New chemical profiles for the asteroseismology of ZZ Ceti stars. Astrophys J 717:897-907. https://doi.org/10.1088/0004-637X/717/2/897. arXiv:1005.2612

Althaus LG, Córsico AH, Isern J, García-Berro E (2010b) Evolutionary and pulsational properties of white dwarf stars. Astron Astrophys Rev 18:471-566. https://doi.org/10.1007/s00159-010-0033-1. arXiv: 1007.2659

Althaus LG, Córsico AH, Torres S, Lorén-Aguilar P, Isern J, García-Berro E (2011) The evolution of white dwarfs with a varying gravitational constant. Astron Astrophys 527:A72. https://doi.org/10.1051/ 0004-6361/201015849. arXiv:1101.0986

Althaus LG, García-Berro E, Isern J, Córsico AH, Miller Bertolami MM (2012) New phase diagrams for dense carbon-oxygen mixtures and white dwarf evolution. Astron Astrophys 537:A33. https://doi. org/10.1051/0004-6361/201117902. arXiv:1110.5665

Althaus LG, Miller Bertolami MM, Córsico AH (2013) New evolutionary sequences for extremely lowmass white dwarfs. Homogeneous mass and age determinations and asteroseismic prospects. Astron Astrophys 557:A19. https://doi.org/10.1051/0004-6361/201321868. arXiv:1307.1882

Althaus LG, Camisassa ME, Miller Bertolami MM, Córsico AH, García-Berro E (2015) White dwarf evolutionary sequences for low-metallicity progenitors: the impact of third dredge-up. Astron Astrophys 576:A9. https://doi.org/10.1051/0004-6361/201424922. arXiv:1502.03882

Althaus LG, De Gerónimo F, Córsico A, Torres S, García-Berro E (2017) The evolution of white dwarfs resulting from helium-enhanced, low-metallicity progenitor stars. Astron Astrophys 597:A67. https:// doi.org/10.1051/0004-6361/201629909. arXiv:1611.06191

Angulo C, Arnould M, Rayet M, Descouvemont P, Baye D, Leclercq-Willain C, Coc A, Barhoumi S et al (1999) A compilation of charged-particle induced thermonuclear reaction rates. Nucl Phys A 656:3183. https://doi.org/10.1016/S0375-9474(99)00030-5

Arras P, Townsley DM, Bildsten L (2006) Pulsational instabilities in accreting white dwarfs. Astrophys J Lett 643:L119-L122. https://doi.org/10.1086/505178. arXiv:astro-ph/0604319

Baade D (1992) Observational Aspects of Stellar Seismology. In: Klare G (ed) Reviews in Modern Astronomy. Vol. 5: Variabilities in Stars and Galaxies, pp 125-142, https://doi.org/10.1007/978-3-64277543-7_9

Balona LA (2010) Challenges In Stellar Pulsation. Bentham Science, Sharjah, https://doi.org/10.2174/ 97816080518541100101

Barlow BN, Dunlap BH, Rosen R, Clemens JC (2008) Two new variable hot DQ stars. Astrophys J Lett 688:L95. https://doi.org/10.1086/595584. arXiv:0810.2140

Battich T, Córsico AH, Althaus LG, Miller Bertolami MM (2016) First axion bounds from a pulsating helium-rich white dwarf star. J Cosmol Astropart Phys 8:062. https://doi.org/10.1088/1475-7516/ 2016/08/062. arXiv:1605.07668

Bedin LR, Salaris M, Piotto G, Anderson J, King IR, Cassisi S (2009) The end of the white dwarf cooling sequence in M4: an efficient approach. Astrophys J 697:965-979. https://doi.org/10.1088/0004-637X/ 697/2/965. arXiv:0903.2839

Bedin LR, Salaris M, Anderson J, Cassisi S, Milone AP, Piotto G, King IR, Bergeron P (2015) Hubble Space Telescope observations of the Kepler-field cluster NGC 6819 - I. The bottom of the white dwarf cooling sequence. Mon Not R Astron Soc 448:1779-1788. https://doi.org/10.1093/mnras/stv069. arXiv: 1501.02953

Bell KJ, Hermes JJ, Montgomery MH, Winget DE, Gentile Fusillo NP, Raddi R, Gänsicke BT (2017b) The first six outbursting cool DA white dwarf pulsators. In: Tremblay PE, Gänsicke B, Marsh T (eds) 20th European white dwarf workshop, ASP Conference Series, vol 509. Astronomical Society of the Pacific, San Francisco, p 303, arXiv:1609.09097

Bell KJ (2017) Pulsational oddities at the extremes of the DA white dwarf instability strip. PhD thesis, University of Texas

Bell KJ, Hermes JJ, Bischoff-Kim A, Moorhead S, Montgomery MH, Østensen R, Castanheira BG, Winget DE (2015) KIC 4552982: outbursts and asteroseismology from the longest pseudocontinuous light curve of a ZZ Ceti. Astrophys J 809:14. https://doi.org/10.1088/0004-637X/809/ 1/14. arXiv: 1506.07878 
Bell KJ, Hermes JJ, Montgomery MH, Gentile Fusillo NP, Raddi R, Gänsicke BT, Winget DE, Dennihy E, Gianninas A, Tremblay PE, Chote P, Winget KI (2016) Outbursts in two new cool pulsating DA white dwarfs. Astrophys J 829:82. https://doi.org/10.3847/0004-637X/829/2/82. arXiv:1607.01392

Bell KJ, Gianninas A, Hermes JJ, Winget DE, Kilic M, Montgomery MH, Castanheira BG, Vanderbosch Z, Winget KI, Brown WR (2017a) Pruning the ELM survey: characterizing candidate low-mass white dwarfs through photometric variability. Astrophys J 835:180. https://doi.org/10.3847/1538-4357/835/ 2/180. arXiv:1612.06390

Bell KJ, Hermes JJ, Vanderbosch Z, Montgomery MH, Winget DE, Dennihy E, Fuchs JT, Tremblay PE (2017c) Destroying Aliases from the ground and space: Super-Nyquist ZZ Cetis in K2 long Cadence Data. Astrophys J 851:24. https://doi.org/10.3847/1538-4357/aa9702. arXiv:1710.10273

Bell KJ, Pelisoli I, Kepler SO, Brown WR, Winget DE, Winget KI, Vanderbosch Z, Castanheira BG, Hermes JJ, Montgomery MH, Koester D (2018) The McDonald Observatory search for pulsating sdA stars. Asteroseismic support for multiple populations. Astron Astrophys 617:A6. https://doi.org/10.1051/ 0004-6361/201833279. arXiv:1805.11129

Bellini A, Anderson J, Salaris M, Cassisi S, Bedin LR, Piotto G, Bergeron P (2013) A double white-dwarf cooling sequence in $\omega$ Centauri. Astrophys J Lett 769:L32. https://doi.org/10.1088/2041-8205/769/ 2/L32. arXiv:1305.0265

Bernstein J, Ruderman M, Feinberg G (1963) Electromagnetic properties of the neutrino. Phys Rev 132:1227-1233. https://doi.org/10.1103/PhysRev.132.1227

Biesiada M, Malec B (2002) White dwarf cooling and large extra dimensions. Phys Rev D 65(4):043008. https://doi.org/10.1103/PhysRevD.65.043008. arXiv:astro-ph/0109545

Biesiada M, Malec B (2003) White dwarf constraints on exotic physics. Astrophys Space Sci 283:601-606. https://doi.org/10.1023/A:1022582802326

Bischoff-Kim A, Montgomery MH, Winget DE (2008a) Fine grid asteroseismology of G117-B15A and R548. Astrophys J 675:1505-1511. https://doi.org/10.1086/527287. arXiv:0711.2039

Bischoff-Kim A, Montgomery MH, Winget DE (2008b) Strong limits on the DFSZ axion mass with G117B15A. Astrophys J 675:1512-1517. https://doi.org/10.1086/526398. arXiv:0711.2041

Bischoff-Kim A, Østensen RH, Hermes JJ, Provencal JL (2014) Seven-period asteroseismic fit of the Kepler DBV. Astrophys J 794:39. https://doi.org/10.1088/0004-637X/794/1/39

Bischoff-Kim A, Provencal JL, Bradley PA, Montgomery MH, Shipman HL, Harrold ST, Howard B, Strickland W et al (2019) GD358: three decades of observations for the in-depth asteroseismology of a DBV star. Astrophys J 871:13. https://doi.org/10.3847/1538-4357/aae2b1. arXiv:1810.11708

Blöcker T (1995) Stellar evolution of low- and intermediate-mass stars. II. Post-AGB evolution. Astron Astrophys 299:755

Blöcker T (2001) Evolution on the AGB and beyond: on the formation of H-deficient post-AGB stars. Astrophys Space Sci 275:1-14 arXiv:astro-ph/0102135

Blöcker T (1995) Stellar evolution of low and intermediate-mass stars. I. Mass loss on the AGB and its consequences for stellar evolution. Astron Astrophys 297:727

Bognár Z, Paparó M, Córsico AH, Kepler SO, Győrffy Á (2014) Revealing the pulsational properties of the V777 Herculis star KUV 05134+2605 by its long-term monitoring. Astron Astrophys 570:A116. https://doi.org/10.1051/0004-6361/201423757. arXiv:1408.3569

Bognár Z, Paparó M, Molnár L, Pápics PI, Plachy E, Verebélyi E, Sódor Á (2016) G 207-9 and LP 133-144: light-curve analysis and asteroseismology of two ZZ Ceti stars. Mon Not R Astron Soc 461:4059-4070. https://doi.org/10.1093/mnras/stw1597. arXiv:1606.09506

Bognár Z, Kalup C, Sódor Á, Charpinet S, Hermes JJ (2018a) Searching for new white dwarf pulsators for TESS observations at Konkoly observatory. Mon Not R Astron Soc 478:2676-2685. https://doi.org/ 10.1093/mnras/sty1393. arXiv:1805.10165

Bognár Z, Paparó M, Sódor Á, Jenei DI, Kalup C, Bertone E, Chavez-Dagostino M, Montgomery MH, Győrffy Á, Molnár L, Ollé H, Pápics PI, Plachy E, Verebélyi E (2018b) Wandering near the red edge: photometric observations of three cool ZZ Ceti stars. Mon Not R Astron Soc. https://doi.org/10.1093/ mnras/sty2884, arXiv:1810.09711

Bognár Z, Sódor Á (2016) White dwarf period tables I. Pulsators with hydrogen-dominated atmospheres. Inform Bull Var Stars 6184. https://doi.org/10.22444/IBVS.6184, arXiv: 1610.07470

Borucki WJ, Koch D, Basri G, Batalha N, Brown T, Caldwell D, Caldwell J, Christensen-Dalsgaard J et al (2010) Kepler planet-detection mission: introduction and first results. Science 327:977. https://doi. org/10.1126/science. 1185402 
Bradley PA (1998) Asteroseismological constraints on the structure of the ZZ Ceti stars G117-B15A and R548. Astrophys J Suppl 116:307-319. https://doi.org/10.1086/313102

Bradley PA (2001) Asteroseismological constraints on the structure of the ZZ Ceti stars L19-2 and GD 165. Astrophys J 552:326-339. https://doi.org/10.1086/320454

Brassard P, Fontaine G (2005) Asteroseismology of the crystallized ZZ Ceti star BPM 37093: a different view. Astrophys J 622:572-576. https://doi.org/10.1086/428116

Brassard P, Fontaine G (1997) Recent advances in the theoretical determination of the ZZ Ceti instability strip. In: Isern J, Hernanz M, Garcia-Berro E (eds) White dwarfs, Astrophysics and Space Science Library, 214. Kluwer, Dordrecht, p 451. https://doi.org/10.1007/978-94-011-5542-7-65

Brassard P, Fontaine G (1999) Convective efficiency and the blue edge in ZZ Ceti Stars. In: Gimenez A, Guinan EF, Montesinos B (eds) Stellar structure: theory and test of connective energy transport, ASP Conference Series, vol 173. Astronomical Society of the Pacific, San Francisco, p 329

Brickhill AJ (1991) The pulsations of ZZ Ceti stars. III-the driving mechanism. Mon Not R Astron Soc 251:673-680. https://doi.org/10.1093/mnras/251.4.673

Brocato E, Matteucci F, Mazzitelli I, Tornambe A (1990) Synthetic colors and the chemical evolution of elliptical galaxies. Astrophys J 349:458-470. https://doi.org/10.1086/168330

Brown WR, Kilic M, Allende Prieto C, Kenyon SJ (2010) The ELM survey. I. A complete sample of extremely low-mass white dwarfs. Astrophys J 723:1072-1081. https://doi.org/10.1088/0004-637X/ 723/2/1072. arXiv:1011.3050

Brown WR, Kilic M, Allende Prieto C, Kenyon SJ (2012) The ELM survey. III. A successful targeted survey for extremely low mass white dwarfs. Astrophys J 744:142. https://doi.org/10.1088/0004-637X/744/ 2/142. arXiv:1111.6588

Brown WR, Kilic M, Allende Prieto C, Gianninas A, Kenyon SJ (2013) The ELM survey. V. Merging massive white dwarf binaries. Astrophys J 769:66. https://doi.org/10.1088/0004-637X/769/1/ 66. arXiv: 1304.4248

Brown WR, Gianninas A, Kilic M, Kenyon SJ, Allende Prieto C (2016) The ELM survey. VII. Orbital properties of low-mass white dwarf binaries. Astrophys J 818:155. https://doi.org/10.3847/0004637X/818/2/155. arXiv:1604.04268

Brown WR, Kilic M, Gianninas A (2017a) The physical nature of subdwarf A stars: white dwarf impostors. Astrophys J 839:23. https://doi.org/10.3847/1538-4357/aa67e4. arXiv:1703.07799

Brown WR, Kilic M, Kosakowski A, Gianninas A (2017b) Discovery of a detached, eclipsing 40 minute period double white dwarf binary and a friend: implications for $\mathrm{He}+\mathrm{CO}$ white dwarf mergers. Astrophys J 847:10. https://doi.org/10.3847/1538-4357/aa8724. arXiv:1708.05287

Byrne CM, Jeffery CS (2018) Post-common envelope binary stars, radiative levitation, and blue largeamplitude pulsators. Mon Not R Astron Soc 481:3810-3820. https://doi.org/10.1093/mnras/sty2545. arXiv: 1809.04183

Calamida A, Corsi CE, Bono G, Stetson PB, Prada Moroni P, Degl'Innocenti S, Ferraro I, Iannicola G, Koester D, Pulone L, Monelli M, Amico P, Buonanno R, Caputo F, D’Odorico S, Freyhammer LM, Marchetti E, Nonino M, Romaniello M (2008) On the white dwarf cooling sequence of the globular cluster $\omega$ Centauri. Astrophys J Lett 673:L29. https://doi.org/10.1086/527436. arXiv:0712.0603

Calcaferro LM, Córsico AH, Althaus LG (2016) Asteroseismology of the GW Virginis stars SDSS J0349-0059 and VV 47. Astron Astrophys 589:A40. https://doi.org/10.1051/0004-6361/201527996. arXiv: 1602.06355

Calcaferro LM, Córsico AH, Althaus LG (2017a) Pulsating low-mass white dwarfs in the frame of new evolutionary sequences. IV. The secular rate of period change. Astron Astrophys 600:A73. https://doi. org/10.1051/0004-6361/201630376. arXiv:1701.08880

Calcaferro LM, Córsico AH, Althaus LG (2017b) Pulsating low-mass white dwarfs in the frame of new evolutionary sequences. V. Asteroseismology of ELMV white dwarf stars. Astron Astrophys 607:A33. https://doi.org/10.1051/0004-6361/201731230. arXiv:1708.00482

Calcaferro LM, Córsico AH, Camisassa ME, Althaus LG, Shibahashi H (2017c) Pulsational instability of high-luminosity H-rich pre-white dwarf star. Eur Phys J Web Conf 152:06012. https://doi.org/10. 1051/epjconf/201715206012

Calcaferro LM, Althaus LG, Córsico AH (2018a) The coolest extremely low-mass white dwarfs. Astron Astrophys 614:A49. https://doi.org/10.1051/0004-6361/201732551. arXiv:1802.06753

Calcaferro LM, Córsico AH, Althaus LG, Romero AD, Kepler SO (2018b) Pulsating low-mass white dwarfs in the frame of new evolutionary sequences. VI. Thin H-envelope sequences and asteroseismology of 
ELMV stars revisited. Astron Astrophys 620:A196. https://doi.org/10.1051/0004-6361/201833781. arXiv: 1810.11502

Caloi V (1989) Evolution of extreme horizontal branch stars. Astron Astrophys 221:27-35

Camisassa ME, Córsico AH, Althaus LG, Shibahashi H (2016) Pulsations powered by hydrogen shell burning in white dwarfs. Astron Astrophys 595:A45. https://doi.org/10.1051/0004-6361/201628857. arXiv: 1606.04367

Camisassa ME, Althaus LG, Córsico AH, De Gerónimo FC, Miller Bertolami MM, Novarino ML, Rohrmann RD, Wachlin FC, García-Berro E (2019) The evolution of ultra-massive white dwarfs. Astron Astrophys 625:A87. https://doi.org/10.1051/0004-6361/201833822. arXiv:1807.03894

Campos F, Kepler SO, Bonatto C, Ducati JR (2013) Multichromatic colour-magnitude diagrams of the globular cluster NGC 6366. Mon Not R Astron Soc 433:243-250. https://doi.org/10.1093/mnras/ stt719. arXiv:1307.4499

Campos F, Bergeron P, Romero AD, Kepler SO, Ourique G, Costa JES, Bonatto CJ, Winget DE, Montgomery MH, Pacheco TA, Bedin LR (2016) A comparative analysis of the observed white dwarf cooling sequence from globular clusters. Mon Not R Astron Soc 456:3729-3742. https://doi.org/10.1093/ mnras/stv2911. arXiv:1512.03114

Cantiello M, Mankovich C, Bildsten L, Christensen-Dalsgaard J, Paxton B (2014) Angular momentum transport within evolved low-mass stars. Astrophys J 788:93. https://doi.org/10.1088/0004-637X/ 788/1/93. arXiv:1405.1419

Carrasco JM, Catalán S, Jordi C, Tremblay PE, Napiwotzki R, Luri X, Robin AC, Kowalski PM (2014) Gaia photometry for white dwarfs. Astron Astrophys 565:A11. https://doi.org/10.1051/0004-6361/ 201220596. arXiv:1403.6045

Casewell SL, Dobbie PD, Napiwotzki R, Burleigh MR, Barstow MA, Jameson RF (2009) High-resolution optical spectroscopy of Praesepe white dwarfs. Mon Not R Astron Soc 395:1795-1804. https://doi. org/10.1111/j.1365-2966.2009.14593.x. arXiv:0901.4464

Castanheira BG, Kepler SO (2009) Seismological studies of ZZ Ceti stars - II. Application to the ZZ Ceti class. Mon Not R Astron Soc 396:1709-1731. https://doi.org/10.1111/j.1365-2966.2009.14855.x

Castanheira BG, Kepler SO, Mullally F, Winget DE, Koester D, Voss B, Kleinman SJ, Nitta A, Eisenstein DJ, Napiwotzki R, Reimers D (2006) Discovery of eleven new ZZ Ceti stars. Astron Astrophys 450:227-231. https://doi.org/10.1051/0004-6361:20053500. arXiv:astro-ph/0511804

Castanheira BG, Kepler SO, Kleinman SJ, Nitta A, Fraga L (2010) New developments of the ZZ Ceti instability strip: the discovery of 11 new variables. Mon Not R Astron Soc 405:2561-2569. https:// doi.org/10.1111/j.1365-2966.2010.16633.x. arXiv:1007.0524

Castanheira BG, Kepler SO, Kleinman SJ, Nitta A, Fraga L (2013) Discovery of five new massive pulsating white dwarf stars. Mon Not R Astron Soc 430:50-59. https://doi.org/10.1093/mnras/sts474

Catelan M (2018) The ages of (the oldest) stars. In: Chiappini C, Minchev I, Starkenburg E, Valentini M (eds) Rediscovering Our Galaxy, IAU Symposium, vol 334. Cambridge University Press, pp 11-20. https://doi.org/10.1017/S1743921318000868, arXiv:1709.08656

Catelan M, Smith HA (2015) Pulsating Stars. Wiley-VCH, Weinheim

Charpinet S, Fontaine G, Brassard P, Dorman B (1997) Gravity-mode instabilities in models of post-extreme horizontal branch stars: another class of pulsating stars? Astrophys J Lett 489:L149. https://doi.org/ 10.1086/316792. arXiv:astro-ph/9707319

Charpinet S, Fontaine G, Brassard P (2009) Seismic evidence for the loss of stellar angular momentum before the white-dwarf stage. Nature 461:501-503. https://doi.org/10.1038/nature08307

Charpinet S, Van Grootel V, Fontaine G, Green EM, Brassard P, Randall SK, Silvotti R, Østensen RH, Kjeldsen H, Christensen-Dalsgaard J, Kawaler SD, Clarke BD, Li J, Wohler B (2011) Deep asteroseismic sounding of the compact hot B subdwarf pulsator KIC02697388 from Kepler time series photometry. Astron Astrophys 530:A3. https://doi.org/10.1051/0004-6361/201016412

Choi J, Conroy C, Ting YS, Cargile PA, Dotter A, Johnson BD (2018) Star cluster ages in the Gaia Era. Astrophys J 863:65. https://doi.org/10.3847/1538-4357/aad18c. arXiv:1807.03789

Chote P, Sullivan DJ, Montgomery MH, Provencal JL (2013) Time series photometry of the helium atmosphere pulsating white dwarf EC 04207-4748. Mon Not R Astron Soc 431:520-527. https://doi.org/ 10.1093/mnras/stt180. arXiv: 1412.5683

Constantino T, Campbell SW, Christensen-Dalsgaard J, Lattanzio JC, Stello D (2015) The treatment of mixing in core helium burning models - I. Implications for asteroseismology. Mon Not R Astron Soc 452:123-145. https://doi.org/10.1093/mnras/stv1264. arXiv:1506.01209 
Copi CJ, Davis AN, Krauss LM (2004) New nucleosynthesis constraint on the variation of $G$. Phys Rev Lett 92(17):171301. https://doi.org/10.1103/PhysRevLett.92.171301. arXiv:astro-ph/0311334

Córsico AH (2018) Pulsating white dwarf stars and asteroseismology. In: Bassion Lea (ed) Terceras Jornadas de Astrofísica Estelar, AAA Workshop Series, vol 9. Asociación Argentina de Astronomía, La Plata, pp 13-22. arXiv:1703.00934

Córsico AH, Romero AD, Althaus LG, Pelisoli I, Kepler SO (2018) Blue Large-Amplitude Pulsators (BLAPs): possible origin, evolutionary status, and nature of their pulsations. ArXiv e-prints arXiv: 1809.07451

Córsico AH, Althaus LG (2014a) Pulsating low-mass white dwarfs in the frame of new evolutionary sequences. I. Adiabatic properties. Astron Astrophys 569:A106. https://doi.org/10.1051/0004-6361/ 201424352. arXiv:1408.6708

Córsico AH, Althaus LG (2014b) Short-period g-mode pulsations in low-mass white dwarfs triggered by H-shell burning. Astrophys J Lett 793:L17. https://doi.org/10.1088/2041-8205/793/1/L17. arXiv: 1408.6724

Córsico AH, Althaus LG (2016) Pulsating low-mass white dwarfs in the frame of new evolutionary sequences. II. Nonadiabatic analysis. Astron Astrophys 585:A1. https://doi.org/10.1051/0004-6361/ 201527162. arXiv: 1510.00645

Córsico AH, Benvenuto OG, Althaus LG, Isern J, García-Berro E (2001) The potential of the variable DA white dwarf G117-B15A as a tool for fundamental physics. New Astron 6:197-213. https://doi.org/ 10.1016/S1384-1076(01)00055-0. arXiv:astro-ph/0104103

Córsico AH, García-Berro E, Althaus LG, Isern J (2004) Pulsations of massive ZZ Ceti stars with carbon/oxygen and oxygen/neon cores. Astron Astrophys 427:923-932. https://doi.org/10.1051/00046361:20040416. arXiv:astro-ph/0408238

Córsico AH, Althaus LG, Miller Bertolami MM, Werner K (2007a) Asteroseismological constraints on the pulsating planetary nebula nucleus (PG 1159-type) RX J2117.1+3412. Astron Astrophys 461:10951102. https://doi.org/10.1051/0004-6361:20066452. arXiv:astro-ph/0610420

Córsico AH, Miller Bertolami MM, Althaus LG, Vauclair G, Werner K (2007b) Asteroseismological constraints on the coolest GW Virginis variable star (PG 1159-type) PG 0122+200. Astron Astrophys 475:619-627. https://doi.org/10.1051/0004-6361:20078145. arXiv:0709.0280

Córsico AH, Althaus LG, Kepler SO, Costa JES, Miller Bertolami MM (2008) Asteroseismological measurements on PG 1159-035, the prototype of the GW Virginis variable stars. Astron Astrophys 478:869-881. https://doi.org/10.1051/0004-6361:20078646. arXiv:0712.0795

Córsico AH, Althaus LG, Miller Bertolami MM, García-Berro E (2009a) Asteroseismology of hot pre-white dwarf stars: the case of the DOV stars PG 2131+066 and PG 1707+427, and the PNNV star NGC 1501. Astron Astrophys 499:257-266. https://doi.org/10.1051/0004-6361/200810727. arXiv:0903.3628

Córsico AH, Althaus LG, Miller Bertolami MM, González Pérez JM, Kepler SO (2009b) On the possible existence of short-period g-mode instabilities powered by nuclear-burning shells in post-asymptotic giant branch H-deficient (PG1159-Type) stars. Astrophys J 701:1008-1014. https://doi.org/10.1088/ 0004-637X/701/2/1008. arXiv:0906.2387

Córsico AH, Althaus LG, Kawaler SD, Miller Bertolami MM, García-Berro E, Kepler SO (2011) Probing the internal rotation of pre-white dwarf stars with asteroseismology: the case of PG 0122+200. Mon Not R Astron Soc 418:2519-2526. https://doi.org/10.1111/j.1365-2966.2011.19642.x. arXiv:1108.3359

Córsico AH, Romero AD, Althaus LG, Hermes JJ (2012d) The seismic properties of low-mass Hecore white dwarf stars. Astron Astrophys 547:A96. https://doi.org/10.1051/0004-6361/201220114. arXiv: 1209.5107

Córsico AH, Althaus LG, Miller Bertolami MM, Bischoff-Kim A (2012a) Asteroseismology of the Kepler V777 Herculis variable white dwarf with fully evolutionary models. Astron Astrophys 541:A42. https://doi.org/10.1051/0004-6361/201118736. arXiv:1112.5882

Córsico AH, Althaus LG, Miller Bertolami MM, Romero AD, García-Berro E, Isern J, Kepler SO (2012) The rate of cooling of the pulsating white dwarf star G117-B15A: a new asteroseismological inference of the axion mass. Mon Not R Astron Soc 424:2792-2799. https://doi.org/10.1111/j.1365-2966.2012. 21401.x

Córsico AH, Althaus LG, Romero AD, Mukadam AS, García-Berro E, Isern J, Kepler SO, Corti MA (2012) An independent limit on the axion mass from the variable white dwarf star R548. J Cosmol Astropart Phys 12:010. https://doi.org/10.1088/1475-7516/2012/12/010. arXiv:1211.3389 
Córsico AH, Althaus LG, García-Berro E, Romero AD (2013) An independent constraint on the secular rate of variation of the gravitational constant from pulsating white dwarfs. J Cosmol Astropart Phys 6:032. https://doi.org/10.1088/1475-7516/2013/06/032. arXiv:1306.1864

Córsico AH, Althaus LG, Miller Bertolami MM, Kepler SO, García-Berro E (2014) Constraining the neutrino magnetic dipole moment from white dwarf pulsations. J Cosmol Astropart Phys 8:054. https://doi.org/10.1088/1475-7516/2014/08/054. arXiv:1406.6034

Córsico AH, Althaus LG, Serenelli AM, Kepler SO, Jeffery CS, Corti MA (2016a) Pulsating low-mass white dwarfs in the frame of new evolutionary sequences. III. The pre-ELM white dwarf instability strip. Astron Astrophys 588:A74. https://doi.org/10.1051/0004-6361/201528032. arXiv:1602.03195

Córsico AH, Romero AD, Althaus LG, García-Berro E, Isern J, Kepler SO, Miller Bertolami MM, Sullivan DJ, Chote P (2016) An asteroseismic constraint on the mass of the axion from the period drift of the pulsating DA white dwarf star L19-2. J Cosmol Astropart Phys 7:036. https://doi.org/10.1088/14757516/2016/07/036. arXiv:1605.06458

Corti MA, Kanaan A, Córsico AH, Kepler SO, Althaus LG, Koester D, Sánchez Arias JP (2016) Two new pulsating low-mass pre-white dwarfs or SX Phoenicis stars? Astron Astrophys 587:L5. https://doi. org/10.1051/0004-6361/201527458. arXiv:1602.00142

Costa JES, Kepler SO (2008) The temporal changes of the pulsational periods of the pre-white dwarf PG 1159-035. Astron Astrophys 489:1225-1232. https://doi.org/10.1051/0004-6361:20079118. arXiv:0807.5137

Costa JES, Kepler SO, Winget DE, O'Brien MS, Kawaler SD, Costa AFM, Giovannini O, Kanaan A et al (2008) The pulsation modes of the pre-white dwarf PG 1159-035. Astron Astrophys 477:627-640. https://doi.org/10.1051/0004-6361:20053470. arXiv:0711.2244

Cox JP (1980) Theory of stellar pulsation. Princeton University Press, Princeton

Cox AN, Starrfield SG, Kidman RB, Dean Pesnell W (1987) Pulsations of white dwarf stars with thick hydrogen or helium surface layers. Astrophys J 317:303-324. https://doi.org/10.1086/165278

Cukanovaite E, Tremblay PE, Freytag B, Ludwig HG, Bergeron P (2018) Pure-helium 3D model atmospheres of white dwarfs. Mon Not R Astron Soc 481:1522-1537. https://doi.org/10.1093/mnras/ sty2383. arXiv: 1809.00590

Cummings JD, Kalirai JS, Tremblay PE, Ramirez-Ruiz E, Choi J (2018) The white dwarf initial-final mass relation for progenitor stars from 0.85 to $7.5 M_{\odot}$. Astrophys J 866:21. https://doi.org/10.3847/15384357/aadfd6. arXiv:1809.01673

Curd B, Gianninas A, Bell KJ, Kilic M, Romero AD, Allende Prieto C, Winget DE, Winget KI (2017) Four new massive pulsating white dwarfs including an ultramassive DAV. Mon Not R Astron Soc 468:239-249. https://doi.org/10.1093/mnras/stx320. arXiv:1702.03343

Dan M, Rosswog S, Brüggen M, Podsiadlowski P (2014) The structure and fate of white dwarf merger remnants. Mon Not R Astron Soc 438:14-34. https://doi.org/10.1093/mnras/stt1766. arXiv:1308.1667

D’Antona F, Mazzitelli I (1990) Cooling of white dwarfs. Annu Rev Astron Astrophys 28:139-181. https:// doi.org/10.1146/annurev.aa.28.090190.001035

De Gerónimo FC, Althaus LG, Córsico AH, Romero AD, Kepler SO (2017) Asteroseismology of ZZ Ceti stars with fully evolutionary white dwarf models. I. The impact of the uncertainties from prior evolution on the period spectrum. Astron Astrophys 599:A21. https://doi.org/10.1051/0004-6361/201629806. arXiv: 1611.10298

De Gerónimo FC, Althaus LG, Córsico AH, Romero AD, Kepler SO (2018) Asteroseismology of ZZ Ceti stars with full evolutionary white dwarf models. II. The impact of AGB thermal pulses on the asteroseismic inferences of ZZ Ceti stars. Astron Astrophys 613:A46. https://doi.org/10.1051/00046361/201731982. arXiv:1801.10589

De Gerónimo FC, Córsico AH, Althaus LG, Wachlin FC, Camisassa ME (2019a) Pulsation properties of ultra-massive DA white dwarf stars with ONe cores. Astron Astrophys 621:A100. https://doi.org/10. 1051/0004-6361/201833789. arXiv:1807.03810

De Gerónimo FC, Battich T, Miller Bertolami MM, Althaus LG, Córsico AH (2019b) On the recent parametric determination of an asteroseismological model for the DBV star KIC 08626021. Astron Astrophys. arXiv:1908.08449v1

De Marco O, Soker N (2002) A new look at the evolution of Wolf-Rayet central stars of planetary Nebulae. PASP 114:602-611. https://doi.org/10.1086/341691. arXiv:astro-ph/0204230

Deheuvels S, García RA, Chaplin WJ, Basu S, Antia HM, Appourchaux T, Benomar O, Davies GR, Elsworth Y, Gizon L, Goupil MJ, Reese DR, Regulo C, Schou J, Stahn T, Casagrande L, Christensen-Dalsgaard J, Fischer D, Hekker S, Kjeldsen H, Mathur S, Mosser B, Pinsonneault M, Valenti J, Christiansen 
JL, Kinemuchi K, Mullally F (2012) Seismic evidence for a rapidly rotating core in a lower-giantbranch star observed with Kepler. Astrophys J 756:19. https://doi.org/10.1088/0004-637X/756/1/19. arXiv: 1206.3312

den Hartogh JW, Eggenberger P, Hirschi R (2019) Constraining transport of angular momentum in stars. Combining asteroseismic observations of core helium burning stars and white dwarfs. Astron Astrophys 622:A187. https://doi.org/10.1051/0004-6361/201834330. arXiv:1902.04293

Dine M, Fischler W, Srednicki M (1981) A simple solution to the strong CP problem with a harmless axion. Phys Lett B 104:199-202. https://doi.org/10.1016/0370-2693(81)90590-6

Dolez N, Vauclair G (1981) Gravity modes instability in DA white dwarfs. Astron Astrophys 102:375-385

Dufour P, Béland S, Fontaine G, Chayer P, Bergeron P (2011) Taking advantage of the COS time-tag capability: observations of pulsating Hot DQ white dwarfs and discovery of a new one. Astrophys J Lett 733:L19. https://doi.org/10.1088/2041-8205/733/2/L19. arXiv:1104.2543

Dunlap BH, Barlow BN, Clemens JC (2010) A new small-amplitude variable Hot DQ white dwarf. Astrophys J Lett 720:L159-L163. https://doi.org/10.1088/2041-8205/720/2/L159. arXiv:1007.5293

Dupret MA, Quirion PO, Fontaine G, Brassard P, Grigahcène A (2008) Time-dependent convection study of the driving mechanism in the DBV white dwarfs. J Phys Conf Ser 118:012051. https://doi.org/10. $1088 / 1742-6596 / 118 / 1 / 012051$

Dupuis CM (2018) A Search for Variability in C-rich DQ White Dwarfs. Master's thesis, Texas A\&M University-Commerce

Dziembowski W (1982) Nonlinear mode coupling in oscillating stars. I. Second order theory of the coherent mode coupling. Acta Astron 32:147-171

Dziembowski W, Koester D (1981) Excitation of gravity modes in white dwarfs with chemically stratified envelopes. Astron Astrophys 97:16-26

Ekström S, Georgy C, Eggenberger P, Meynet G, Mowlavi N, Wyttenbach A, Granada A, Decressin T, Hirschi R, Frischknecht U, Charbonnel C, Maeder A (2012) Grids of stellar models with rotation. I. Models from 0.8 to $120 M_{\odot}$ at solar metallicity $(\mathrm{Z}=0.014)$. Astron Astrophys 537:A146. https://doi. org/10.1051/0004-6361/201117751. arXiv:1110.5049

Farihi J (2016) Circumstellar debris and pollution at white dwarf stars. New Astron Rev 71:9-34. https:// doi.org/10.1016/j.newar.2016.03.001. arXiv:1604.03092

Faulkner J (1972) On the nature of the horizontal branch. II. Extremely blue Halo stars: a theoretical viewpoint. Astrophys J 173:401. https://doi.org/10.1086/151430

Fields CE, Farmer R, Petermann I, Iliadis C, Timmes FX (2016) Properties of carbon-oxygen white dwarfs from Monte Carlo stellar models. Astrophys J 823:46. https://doi.org/10.3847/0004-637X/823/1/46. arXiv: 1603.06666

Fontaine G, Brassard P (2008) The pulsating white dwarf stars. PASP 120:1043-1096. https://doi.org/10. $1086 / 592788$

Fontaine G, Brassard P, Bergeron P (2001) The potential of white dwarf cosmochronology. PASP 113:409_ 435. https://doi.org/10.1086/319535

Fontaine G, Brassard P, Charpinet S (2013) The angular momentum of isolated white dwarfs. Eur Phys J Web Conf 43:05011. https://doi.org/10.1051/epjconf/20134305011

Fontaine G, Istrate A, Gianninas A, Brassard P, Van Grootel V (2017) Making Sense Out of Pulsating Pre-ELM and ELM White Dwarfs. In: Tremblay PE, Gaensicke B, Marsh T (eds) 20th European White Dwarf Workshop, ASP Conference Series, vol 509. Astronomical Society of the Pacific, San Francisco, p 347

Fu JN, Dolez N, Vauclair G, Fox-Machado L, Kim SL, Li C, Chen L, Alvarez M, Su J, Charpinet S, Chevreton M, Michel R, Yang XH, Li Y, Zhang YP, Molnar L, Plachy E (2013) Asteroseismology of the ZZ Ceti star HS 0507+0434B. Mon Not R Astron Soc 429:1585-1595. https://doi.org/10.1093/ mnras/sts438. arXiv: 1211.6226

Fu JN, Vauclair G, Su J, Fox Machado L, Colas F, Kim SL, Cang TQ, Li C, Niu HB, Xue HF, Li Y, Jiang XJ, Michel R, Alvarez M, Dolez N, Ma L, Esamdin A, Liu JZ (2019) Precise determination of stellar parameters of the ZZ Ceti and DAZ white dwarf GD 133 through asteroseismology. Mon Not R Astron Soc 486(3):3560-3568. https://doi.org/10.1093/mnras/stz1088. arXiv:1904.07586

Fujikawa K, Shrock RE (1980) Magnetic Moment of a Massive Neutrino and Neutrino-Spin Rotation. Phys Rev Lett 45:963-966. https://doi.org/10.1103/PhysRevLett.45.963

Fuller J, Lai D (2011) Tidal excitations of oscillation modes in compact white dwarf binaries - I. Linear theory. Mon Not R Astron Soc 412:1331-1340. https://doi.org/10.1111/j.1365-2966.2010.18017.x. arXiv:1009.3316 
Fuller J, Lai D (2012) Dynamical tides in compact white dwarf binaries: tidal synchronization and dissipation. Mon Not R Astron Soc 421:426-445. https://doi.org/10.1111/j.1365-2966.2011.20320.x. arXiv: 1108.4910

Gaia Collaboration, Babusiaux C, van Leeuwen F, Barstow MA, Jordi C, Vallenari A, Bossini D, Bressan A, Cantat-Gaudin T, van Leeuwen M, et al (2018a) Gaia Data Release 2. Observational Hertzsprung-Russell diagrams. Astron Astrophys 616:A10, https://doi.org/10.1051/0004-6361/ 201832843, arXiv:1804.09378

Gaia Collaboration, Brown AGA, Vallenari A, Prusti T, de Bruijne JHJ, Babusiaux C, Bailer-Jones CAL, Biermann M, Evans DW, Eyer L, et al (2018b) Gaia Data Release 2. Summary of the contents and survey properties. Astron Astrophys 616:A1, https://doi.org/10.1051/0004-6361/201833051, arXiv: 1804.09365

Gänsicke BT, Koester D, Girven J, Marsh TR, Steeghs D (2010) Two white dwarfs with oxygen-rich atmospheres. Science 327:188. https://doi.org/10.1126/science.1180228. arXiv:0911.2246

Gänsicke BT, Koester D, Farihi J, Girven J, Parsons SG, Breedt E (2012) The chemical diversity of exoterrestrial planetary debris around white dwarfs. Mon Not R Astron Soc 424:333-347. https://doi.org/ 10.1111/j.1365-2966.2012.21201.x. arXiv:1205.0167

García-Berro E, Oswalt TD (2016) The white dwarf luminosity function. New Astron Rev 72:1-22. https:// doi.org/10.1016/j.newar.2016.08.001. arXiv:1608.02631

Garcia-Berro E, Kubyshin Y, Loren-Aguilar P, Isern J (2006) The variation of the gravitational constant inferred from the hubble diagram of type Ia supernovae. Int J Mod Phys D 15:1163-1174. https://doi. org/10.1142/S0218271806008772. arXiv:gr-qc/0512164

García-Berro E, Isern J, Kubyshin YA (2007) Astronomical measurements and constraints on the variability of fundamental constants. Astron Astrophys Rev 14:113-170. https://doi.org/10.1007/s00159-0060004-8

García-Berro E, Torres S, Althaus LG, Renedo I, Lorén-Aguilar P, Córsico AH, Rohrmann RD, Salaris M, Isern J (2010) A white dwarf cooling age of 8 Gyr for NGC 6791 from physical separation processes. Nature 465:194-196. https://doi.org/10.1038/nature09045. arXiv:1005.2272

García-Berro E, Lorén-Aguilar P, Torres S, Althaus LG, Isern J (2011) An upper limit to the secular variation of the gravitational constant from white dwarf stars. J Cosmol Astropart Phys 5:021. https://doi.org/ 10.1088/1475-7516/2011/05/021. arXiv:1105.1992

García-Berro E, Lorén-Aguilar P, Aznar-Siguán G, Torres S, Camacho J, Althaus LG, Córsico AH, Külebi B, Isern J (2012) Double degenerate mergers as progenitors of high-field magnetic white dwarfs. Astrophys J 749:25. https://doi.org/10.1088/0004-637X/749/1/25. arXiv:1202.0461

Gauss CF (1809) Theoria motvs corporvm coelestivm in sectionibvs conicis solem ambientivm. Svmtibvs F. Perthes et I. H. Besser, Hambvrgi

Gautschy A, Althaus LG, Saio H (2005) On the excitation of PG 1159-type pulsations. Astron Astrophys 438:1013-1020. https://doi.org/10.1051/0004-6361:20042486. arXiv:astro-ph/0504495

Gentile Fusillo NP, Tremblay PE, Gänsicke BT, Manser CJ, Cunningham T, Cukanovaite E, Hollands M, Marsh T, Raddi R, Jordan S, Toonen S, Geier S, Barstow M, Cummings JD (2019) A Gaia data release 2 catalogue of white dwarfs and a comparison with SDSS. Mon Not R Astron Soc 482:4570-4591. https://doi.org/10.1093/mnras/sty3016. arXiv:1807.03315

Giammichele N, Charpinet S, Brassard P, Fontaine G (2017a) The potential of asteroseismology for probing the core chemical stratification in white dwarf stars. Astron Astrophys 598:A109. https://doi.org/10. 1051/0004-6361/201629935. arXiv:1611.05071

Giammichele N, Charpinet S, Fontaine G, Brassard P (2017b) Toward high-precision seismic studies of white dwarf stars: parametrization of the core and tests of accuracy. Astrophys J 834:136. https://doi. org/10.3847/1538-4357/834/2/136. arXiv:1610.06036

Giammichele N, Charpinet S, Fontaine G, Brassard P, Green EM, Van Grootel V, Bergeron P, Zong W, Dupret MA (2018) A large oxygen-dominated core from the seismic cartography of a pulsating white dwarf. Nature 554:73-76. https://doi.org/10.1038/nature25136

Gianninas A, Bergeron P, Dupuis J, Ruiz MT (2010) Spectroscopic analysis of hot, hydrogen-rich white dwarfs: the presence of metals and the Balmer-line problem. Astrophys J 720:581-602. https://doi. org/10.1088/0004-637X/720/1/581

Gianninas A, Bergeron P, Ruiz MT (2011) A spectroscopic survey and analysis of bright, hydrogen-rich white dwarfs. Astrophys J 743:138. https://doi.org/10.1088/0004-637X/743/2/138. arXiv:1109.3171 
Gianninas A, Dufour P, Kilic M, Brown WR, Bergeron P, Hermes JJ (2014) Precise atmospheric parameters for the shortest-period binary white dwarfs: gravitational waves, metals, and pulsations. Astrophys J 794:35. https://doi.org/10.1088/0004-637X/794/1/35. arXiv:1408.3118

Gianninas A, Kilic M, Brown WR, Canton P, Kenyon SJ (2015) The ELM Survey. VI. Eleven New Double Degenerates. Astrophys. J. 812:167. https://doi.org/10.1088/0004-637X/812/2/167. arXiv: 1509.07134

Gianninas A, Curd B, Fontaine G, Brown WR, Kilic M (2016) Discovery of three pulsating, mixedatmosphere, extremely low-mass white dwarf precursors. Astrophys J Lett 822:L27. https://doi.org/ 10.3847/2041-8205/822/2/L27. arXiv:1604.04621

Giannotti M (2017) Axion searches: exciting times. Nat Phys 13:530-531. https://doi.org/10.1038/ nphys4139

Giannotti M, Irastorza IG, Redondo J, Ringwald A, Saikawa K (2017) Stellar recipes for axion hunters. J Cosmol Astropart Phys 10:010. https://doi.org/10.1088/1475-7516/2017/10/010. arXiv:1708.02111

Gilliland RL, Jenkins JM, Borucki WJ, Bryson ST, Caldwell DA, Clarke BD, Dotson JL, Haas MR, Hall J, Klaus T, Koch D, McCauliff S, Quintana EV, Twicken JD, van Cleve JE (2010) Initial characteristics of Kepler short cadence data. Astrophys J Lett 713:L160-L163. https://doi.org/10.1088/2041-8205/ 713/2/L160. arXiv:1001.0142

Goldreich P, Wu Y (1999) Gravity modes in ZZ Ceti stars. I. Quasi-adiabatic analysis of overstability. Astrophys J 511:904-915. https://doi.org/10.1086/306705. arXiv:astro-ph/9804305

González Pérez JM, Solheim JE, Kamben R (2006) A search for photometric variability of hydrogendeficient planetary-nebula nuclei. Astron Astrophys 454:527-536. https://doi.org/10.1051/00046361:20053468

Greggio L, Renzini A (1990) Clues on the hot star content and the ultraviolet output of elliptical galaxies. Astrophys J 364:35-64. https://doi.org/10.1086/169384

Greiss S, Hermes JJ, Gänsicke BT, Steeghs DTH, Bell KJ, Raddi R, Tremblay PE, Breedt E, Ramsay G, Koester D, Carter PJ, Vanderbosch Z, Winget DE, Winget KI (2016) The search for ZZ Ceti stars in the original Kepler mission. Mon Not R Astron Soc 457:2855-2863. https://doi.org/10.1093/mnras/ stw053. arXiv:1601.01316

Grigahcène A, Dupret MA, Gabriel M, Garrido R, Scuflaire R (2005) Convection-pulsation coupling. I. A mixing-length perturbative theory. Astron Astrophys 434:1055-1062. https://doi.org/10.1051/00046361:20041816

Haft M, Raffelt G, Weiss A (1994) Standard and nonstandard plasma neutrino emission revisited. Astrophys J 425:222-230. https://doi.org/10.1086/173978. arXiv:astro-ph/9309014

Han Z, Tout CA, Eggleton PP (2000) Low- and intermediate-mass close binary evolution and the initialfinal mass relation. Mon Not R Astron Soc 319:215-222. https://doi.org/10.1046/j.1365-8711.2000. 03839.x. arXiv:astro-ph/0010269

Hansen B (2004) The astrophysics of cool white dwarfs. Phys Rep 399:1-70. https://doi.org/10.1016/j. physrep.2004.07.001

Hansen BMS, Liebert J (2003) Cool white dwarfs. Annu Rev Astron Astrophys 41:465-515. https://doi. org/10.1146/annurev.astro.41.081401.155117

Hansen CJ, Winget DE, Kawaler SD (1985) Upper and lower bounds of periods in variable white dwarfs. Astrophys J 297:544-547. https://doi.org/10.1086/163549

Hansen BMS, Richer H, Kalirai J, Goldsbury R, Frewen S, Heyl J (2015) Constraining neutrino cooling using the hot white dwarf luminosity function in the globular cluster 47 Tucanae. Astrophys J 809(2):141. https://doi.org/10.1088/0004-637X/809/2/141. arXiv:1507.05665

Hermes JJ, Mullally F, Østensen RH, Williams KA, Telting J, Southworth J, Bloemen S, Howell SB, Everett M, Winget DE (2011) Discovery of a ZZ Ceti in the Kepler mission field. Astrophys J Lett 741:L16. https://doi.org/10.1088/2041-8205/741/1/L16. arXiv:1109.6023

Hermes JJ, Montgomery MH, Winget DE, Brown WR, Kilic M, Kenyon SJ (2012) SDSS J184037.78+642312.3: the first pulsating extremely low mass white dwarf. Astrophys J Lett 750:L28. https://doi.org/10.1088/2041-8205/750/2/L28. arXiv:1204.1338

Hermes JJ, Kepler SO, Castanheira BG, Gianninas A, Winget DE, Montgomery MH, Brown WR, Harrold ST (2013a) Discovery of an ultramassive pulsating white dwarf. Astrophys J Lett 771:L2. https://doi. org/10.1088/2041-8205/771/1/L2. arXiv:1306.4024

Hermes JJ, Montgomery MH, Gianninas A, Winget DE, Brown WR, Harrold ST, Bell KJ, Kenyon SJ, Kilic M, Castanheira BG (2013b) A new class of pulsating white dwarf of extremely low mass: the fourth 
and fifth members. Mon Not R Astron Soc 436:3573-3580. https://doi.org/10.1093/mnras/stt1835. arXiv: 1310.0013

Hermes JJ, Montgomery MH, Mullally F, Winget DE, Bischoff-Kim A (2013c) A new timescale for period change in the pulsating DA white dwarf WD 0111+0018. Astrophys J 766:42. https://doi.org/10.1088/ 0004-637X/766/1/42. arXiv:1302.1875

Hermes JJ, Montgomery MH, Winget DE, Brown WR, Gianninas A, Kilic M, Kenyon SJ, Bell KJ, Harrold ST (2013d) Discovery of pulsations, including possible pressure modes, in two new extremely low mass, He-core white dwarfs. Astrophys J 765:102. https://doi.org/10.1088/0004-637X/765/2/102. arXiv: 1211.1022

Hermes JJ, Montgomery MH, Bell KJ, Chote P, Gänsicke BT, Kawaler SD, Clemens JC, Dunlap BH, Winget DE, Armstrong DJ (2015) A second case of outbursts in a pulsating white dwarf observed by Kepler. Astrophys J Lett 810:L5. https://doi.org/10.1088/2041-8205/810/1/L5. arXiv:1507.06319

Hermes JJ, Gänsicke BT, Kawaler SD, Greiss S, Tremblay PE, Gentile Fusillo NP, Raddi R, Fanale SM, Bell KJ, Dennihy E, Fuchs JT, Dunlap BH, Clemens JC, Montgomery MH, Winget DE, Chote P, Marsh TR, Redfield S (2017a) White dwarf rotation as a function of mass and a dichotomy of mode line widths: Kepler observations of 27 pulsating DA white dwarfs through K2 campaign 8. Astrophys J Suppl 232:23. https://doi.org/10.3847/1538-4365/aa8bb5. arXiv:1709.07004

Hermes JJ, Kawaler SD, Bischoff-Kim A, Provencal JL, Dunlap BH, Clemens JC (2017b) A deep test of radial differential rotation in a helium-atmosphere white dwarf. I. Discovery of pulsations in PG 0112+104. Astrophys J 835:277. https://doi.org/10.3847/1538-4357/835/2/277. arXiv:1612.07807

Herwig F (2005) Evolution of asymptotic giant branch stars. Annu Rev Astron Astrophys 43:435-479. https://doi.org/10.1146/annurev.astro.43.072103.150600

Herwig F, Blöcker T, Langer N, Driebe T (1999) On the formation of hydrogen-deficient post-AGB stars. Astron Astrophys 349:L5-L8 arXiv:astro-ph/9908108

Hofmann F, Müller J, Biskupek L (2010) Lunar laser ranging test of the Nordtvedt parameter and a possible variation in the gravitational constant. Astron Astrophys 522:L5. https://doi.org/10.1051/0004-6361/ 201015659

Höfner S, Olofsson H (2018) Mass loss of stars on the asymptotic giant branch. Mechanisms, models and measurements. Astron Astrophys Rev 26:1. https://doi.org/10.1007/s00159-017-0106-5

Hollands MA, Gänsicke BT, Koester D (2018) Cool DZ white dwarfs II: compositions and evolution of old remnant planetary systems. Mon Not R Astron Soc 477:93-111. https://doi.org/10.1093/mnras/ sty592. arXiv: 1801.07714

Hoof S, Kahlhoefer F, Scott P, Weniger C, White M (2019) Axion global fits with Peccei-Quinn symmetry breaking before inflation using GAMBIT. JHEP 3:191. https://doi.org/10.1007/JHEP03(2019)191. arXiv: 1810.07192

Horowitz CJ, Schneider AS, Berry DK (2010) Crystallization of carbon-oxygen mixtures in white dwarf stars. Phys Rev Lett 104(23):231101. https://doi.org/10.1103/PhysRevLett.104.231101. arXiv: 1005.2441

Howell SB, Sobeck C, Haas M, Still M, Barclay T, Mullally F, Troeltzsch J, Aigrain S, Bryson ST, Caldwell D, Chaplin WJ, Cochran WD, Huber D, Marcy GW, Miglio A, Najita JR, Smith M, Twicken JD, Fortney JJ (2014) The K2 mission: characterization and early results. PASP 126:398. https://doi.org/ 10.1086/676406. arXiv:1402.5163

Hu J, Webb JK, Ayres TR, Bainbridge MB, Barrow JD, Barstow MA, Berengut JC, Carswell RF, Dzuba VA, Flambaum VV, Holberg JB, Lee CC, Preval SP, Reindl N, Tchang-Brillet WÜL (2019) Constraining the magnetic field on white dwarf surfaces; Zeeman effects and fine structure constant variation. Mon Not R Astron Soc 485:5050-5058. https://doi.org/10.1093/mnras/stz739. arXiv:1812.11480

Iben I Jr, MacDonald J (1995) The Born Again AGB Phenomenon. In: Koester D, Werner K (eds) White Dwarfs, Springer, Berlin, Lecture Notes in Physics, vol 443, pp 48-57. https://doi.org/10.1007/3-54059157-5_173

Iben I Jr (1984) On the frequency of planetary nebula nuclei powered by helium burning and on the frequency of white dwarfs with hydrogen-deficient atmospheres. Astrophys J 277:333-354. https://doi.org/10. $1086 / 161700$

Iben I Jr, MacDonald J (1986) The effects of diffusion due to gravity and due to composition gradients on the rate of hydrogen burning in a cooling degenerate dwarf. II. Dependence on initial metallicity and on buffer mass. Astrophys J 301:164-176. https://doi.org/10.1086/163884

Irastorza IG, Redondo J (2018) New experimental approaches in the search for axion-like particles. Prog Part Nuclear Phys 102:89-159. https://doi.org/10.1016/j.ppnp.2018.05.003. arXiv:1801.08127 
Isern J, Hernanz M, Garcia-Berro E (1992) Axion cooling of white dwarfs. Astrophys J Lett 392:L23-L25. https://doi.org/10.1086/186416

Isern J, García-Berro E, Torres S, Catalán S (2008) Axions and the cooling of white dwarf stars. Astrophys J Lett 682:L109. https://doi.org/10.1086/591042. arXiv:0806.2807

Isern J, Catalán S, García-Berro E, Torres S (2009) Axions and the white dwarf luminosity function. J Phys Conf Ser 172:012005. https://doi.org/10.1088/1742-6596/172/1/012005. arXiv:0812.3043

Isern J, García-Berro E, Torres S, Cojocaru R, Catalán S (2018) Axions and the luminosity function of white dwarfs: the thin and thick discs, and the halo. Mon Not R Astron Soc 478:2569-2575. https:// doi.org/10.1093/mnras/sty1162. arXiv:1805.00135

Istrate AG, Fontaine G, Gianninas A, Grassitelli L, Marchant P, Tauris TM, Langer N (2016a) Asteroseismic test of rotational mixing in low-mass white dwarfs. Astron Astrophys 595:L12. https://doi.org/10. 1051/0004-6361/201629876. arXiv:1610.08513

Istrate AG, Marchant P, Tauris TM, Langer N, Stancliffe RJ, Grassitelli L (2016b) Models of low-mass helium white dwarfs including gravitational settling, thermal and chemical diffusion, and rotational mixing. Astron Astrophys 595:A35. https://doi.org/10.1051/0004-6361/201628874. arXiv: 1606.04947

Istrate AG, Fontaine G, Heuser C (2017) A model of the pulsating extremely low-mass white dwarf precursor WASP 0247-25B. Astrophys J 847:130. https://doi.org/10.3847/1538-4357/aa8958. arXiv: 1708.09388

Ivanova N, Justham S, Chen X, De Marco O, Fryer CL, Gaburov E, Ge H, Glebbeek E, Han Z, Li XD, Lu G, Marsh T, Podsiadlowski P, Potter A, Soker N, Taam R, Tauris TM, van den Heuvel EPJ, Webbink RF (2013) Common envelope evolution: where we stand and how we can move forward. Astron Astrophys Rev 21:59. https://doi.org/10.1007/s00159-013-0059-2. arXiv:1209.4302

Jeffery CS, Saio H (2013) Pulsation in extremely low mass helium stars. Mon Not R Astron Soc 435:885892. https://doi.org/10.1093/mnras/stt1360

Kanaan A, Kepler SO, Giovannini O, Diaz M (1992) The discovery of a new DAV star using IUE temperature determination. Astrophys J Lett 390:L89-L91. https://doi.org/10.1086/186379

Kanaan A, Nitta A, Winget DE, Kepler SO, Montgomery MH, Metcalfe TS, Oliveira H, Fraga L et al (2005) Whole Earth Telescope observations of BPM 37093: a seismological test of crystallization theory in white dwarfs. Astron Astrophys 432:219-224. https://doi.org/10.1051/0004-6361:20041125. arXiv:astro-ph/0411199

Karakas AI, Lattanzio JC (2014) The Dawes review 2: nucleosynthesis and stellar yields of low- and intermediate-mass single stars. PASA 31:e030. https://doi.org/10.1017/pasa.2014.21. arXiv: 1405.0062

Kawaler SD (2015) Rotation of White Dwarf Stars. In: Dufour P, Bergeron P, Fontaine G (eds) 19th European Workshop on White Dwarfs, ASP Conference Series, vol 493. Astronomical Society of the Pacific, San Francisco, p 65. arXiv:1410.6934

Kawaler SD (1988) The hydrogen shell game: pulsational instabilities in hydrogen shell-burning planetary nebula nuclei. Astrophys J 334:220-228. https://doi.org/10.1086/166832

Kawaler SD, Winget DE, Hansen CJ, Iben I Jr (1986) The helium shell game-Nonradial g-mode instabilities in hydrogen-deficient planetary nebula nuclei. Astrophys J Lett 306:L41-L44. https://doi.org/10.1086/ 184701

Kawaler SD, Sekii T, Gough D (1999) Prospects for measuring differential rotation in white dwarfs through asteroseismology. Astrophys J 516:349-365. https://doi.org/10.1086/307087. arXiv:astro-ph/9811286

Kepler SO (2012) White Dwarf Stars: Pulsations and Magnetism. In: Shibahashi H, Takata M, Lynas-Gray AE (eds) Progress in Solar/Stellar Physics with Helio- and Asteroseismology, ASP Conference Series, vol 462. Astronomical Society of the Pacific, San Francisco, p 322

Kepler SO, Bradley PA (1995) Structure and evolution of white dwarfs. Baltic Astron 4:166-220. https:// doi.org/10.1515/astro-1995-0213

Kepler SO, Romero AD (2017) Pulsating white dwarfs. Eur Phys J Web Conf 152:01011. https://doi.org/ 10.1051/epjconf/201715201011. arXiv:1706.07020

Kepler SO, Nather RE, Winget DE, Nitta A, Kleinman SJ, Metcalfe T, Sekiguchi K, Jiang X, Sullivan D, Sullivan T, Janulis R, Meistas E, Kalytis R, Krzesinski J, Ogoza W, Zola S, O’Donoghue D, Romero-Colmenero E, Martinez P, Dreizler S, Deetjen J, Nagel T, Schuh SL, Vauclair G, Ning FJ, Chevreton M, Solheim JE, Gonzalez Perez JM, Johannessen F, Kanaan A, Costa JE, Murillo Costa AF, Wood MA, Silvestri N, Ahrens TJ, Jones AK, Collins AE, Boyer M, Shaw JS, Mukadam A, 
Klumpe EW, Larrison J, Kawaler S, Riddle R, Ulla A, Bradley P (2003) The everchanging pulsating white dwarf GD358. Astron Astrophys 401:639-654. https://doi.org/10.1051/0004-6361:20030105. arXiv:astro-ph/0301477

Kepler SO, Costa JES, Castanheira BG, Winget DE, Mullally F, Nather RE, Kilic M, von Hippel T, Mukadam AS, Sullivan DJ (2005) Measuring the evolution of the most stable optical clock G 117-B15A. Astrophys J 634:1311-1318. https://doi.org/10.1086/497002. arXiv:astro-ph/0507487

Kepler SO, Fraga L, Winget DE, Bell K, Córsico AH, Werner K (2014) Discovery of a new PG 1159 (GW Vir) pulsator. Mon Not R Astron Soc 442:2278-2281. https://doi.org/10.1093/mnras/stu1019. arXiv: 1405.5075

Kepler SO, Pelisoli I, Koester D, Ourique G, Kleinman SJ, Romero AD, Nitta A, Eisenstein DJ, Costa JES, Külebi B, Jordan S, Dufour P, Giommi P, Rebassa-Mansergas A (2015) New white dwarf stars in the Sloan digital sky survey data release 10. Mon Not R Astron Soc 446:4078-4087. https://doi.org/10. 1093/mnras/stu2388. arXiv:1411.4149

Kepler SO, Koester D, Ourique G (2016a) A white dwarf with an oxygen atmosphere. Science 352:67-69. https://doi.org/10.1126/science.aad6705

Kepler SO, Pelisoli I, Koester D, Ourique G, Romero AD, Reindl N, Kleinman SJ, Eisenstein DJ, Valois ADM, Amaral LA (2016b) New white dwarf and subdwarf stars in the Sloan digital sky survey data release 12. Mon Not R Astron Soc 455:3413-3423. https://doi.org/10.1093/mnras/stv2526. arXiv: 1510.08409

Kepler SO, Pelisoli I, Koester D, Reindl N, Geier S, Romero AD, Ourique G, Oliveira CdP, Amaral LA (2019) White dwarf and subdwarf stars in the Sloan digital sky survey data release 14. Mon Not R Astron Soc 486:2169-2183. https://doi.org/10.1093/mnras/stz960. arXiv:1904.01626

Kilic M, Brown WR, Allende Prieto C, Agüeros MA, Heinke C, Kenyon SJ (2011) The ELM survey. II. Twelve binary white dwarf merger systems. Astrophys J 727:3. https://doi.org/10.1088/0004-637X/ 727/1/3. arXiv: 1011.4073

Kilic M, Brown WR, Allende Prieto C, Kenyon SJ, Heinke CO, Agüeros MA, Kleinman SJ (2012) The ELM survey. IV. 24 white dwarf merger systems. Astrophys J 751:141. https://doi.org/10.1088/0004637X/751/2/141. arXiv:1204.0028

Kilic M, Hermes JJ, Gianninas A, Brown WR (2015) PSR J1738+0333: the first millisecond pulsar + pulsating white dwarf binary. Mon Not R Astron Soc 446:L26-L30. https://doi.org/10.1093/mnrasl/ slu152. arXiv: 1410.4898

Kilic M, Munn JA, Harris HC, von Hippel T, Liebert JW, Williams KA, Jeffery E, DeGennaro S (2017) The ages of the thin disk, thick disk, and the Halo from nearby white dwarfs. Astrophys J 837:162. https://doi.org/10.3847/1538-4357/aa62a5. arXiv:1702.06984

Kilic M, Hermes JJ, Córsico AH, Kosakowski A, Brown WR, Antoniadis J, Calcaferro LM, Gianninas A, Althaus LG, Green MJ (2018) A refined search for pulsations in white dwarf companions to millisecond pulsars. Mon Not R Astron Soc 479:1267-1272. https://doi.org/10.1093/mnras/sty1546. arXiv: 1806.03650

Kim JE, Carosi G (2010) Axions and the strong CP problem. Rev Mod Phys 82:557-601. https://doi.org/ 10.1103/RevModPhys.82.557. arXiv:0807.3125

Kippenhahn R, Weigert A, Weiss A (2012) Stellar Structure and Evolution. Astronomy and Astrophysics Library. Springer, Berlin, Heidelberg. https://doi.org/10.1007/978-3-642-30304-3

Kleinman SJ, Kepler SO, Koester D, Pelisoli I, Peçanha V, Nitta A, Costa JES, Krzesinski J, Dufour P, Lachapelle FR, Bergeron P, Yip CW, Harris HC, Eisenstein DJ, Althaus L, Córsico A (2013) SDSS DR7 white dwarf catalog. Astrophys J Suppl Ser 204:5. https://doi.org/10.1088/0067-0049/204/1/5. arXiv: 1212.1222

Koester D (2002) White dwarfs: recent developments. Astron Astrophys Rev 11:33-66. https://doi.org/10. $1007 / \mathrm{s} 001590100015$

Koester D, Chanmugam G (1990) Physics of white dwarf stars. Rep Progr Phys 53:837-915. https://doi. org/10.1088/0034-4885/53/7/001

Koester D, Kepler SO (2015) DB white dwarfs in the Sloan digital sky survey data release 10 and 12 . Astron Astrophys 583:A86. https://doi.org/10.1051/0004-6361/201527169. arXiv:1509.08244

Koester D, Voss B, Napiwotzki R, Christlieb N, Homeier D, Lisker T, Reimers D, Heber U (2009) Highresolution UVES/VLT spectra of white dwarfs observed for the ESO SN Ia Progenitor Survey. III. DA white dwarfs. Astron Astrophys 505:441-462. https://doi.org/10.1051/0004-6361/200912531. arXiv:0908.2322 
Koester D, Gänsicke BT, Farihi J (2014) The frequency of planetary debris around young white dwarfs. Astron Astrophys 566:A34. https://doi.org/10.1051/0004-6361/201423691. arXiv:1404.2617

Kunz R, Fey M, Jaeger M, Mayer A, Hammer JW, Staudt G, Harissopulos S, Paradellis T (2002) Astrophysical reaction rate of ${ }^{12} \mathrm{C}(\alpha, \gamma){ }^{16} \mathrm{O}$. Astrophys J 567:643-650. https://doi.org/10.1086/338384

Kurtz DW, Shibahashi H, Dhillon VS, Marsh TR, Littlefair SP (2008) A search for a new class of pulsating DA white dwarf stars in the DB gap. Mon Not R Astron Soc 389:1771-1779. https://doi.org/10.1111/ j.1365-2966.2008.13664.x

Kurtz DW, Shibahashi H, Dhillon VS, Marsh TR, Littlefair SP, Copperwheat CM, Gänsicke BT, Parsons SG (2013) Hot DAVs: a probable new class of pulsating white dwarf stars. Mon Not R Astron Soc 432:1632-1639. https://doi.org/10.1093/mnras/stt585

Landolt AU (1968) A new short-period blue variable. Astrophys J 153:151. https://doi.org/10.1086/149645

Laplace PS (1810) Mémoire sur les approximations des formules qui sont fonctions de très-grands nombres, et sur leur application aux probabilités (suite). Mém Acad Sci pp 301-345

Lauffer GR, Romero AD, Kepler SO (2018) New full evolutionary sequences of H- and He-atmosphere massive white dwarf stars using MESA. Mon Not R Astron Soc 480:1547-1562. https://doi.org/10. 1093/mnras/sty1925. arXiv:1807.04774

Lecoanet D, Schwab J, Quataert E, Bildsten L, Timmes FX, Burns KJ, Vasil GM, Oishi JS, Brown BP (2016) Turbulent chemical diffusion in convectively bounded carbon flames. Astrophys J 832:71. https://doi. org/10.3847/0004-637X/832/1/71. arXiv:1603.08921

Ledoux P, Walraven T (1958) Variable stars. Handbuch der Physik 51:353-604

Legendre AM (1806) Nouvelles Methodes pour la determination des Orbites des Cometes. Courcier, Paris

Luan J, Goldreich P (2018) DAVs: red edge and outbursts. Astrophys J 863:82. https://doi.org/10.3847/ 1538-4357/aad0f4. arXiv:1711.06367

Luders G (1954) On the equivalence of invariance under time reversal and under particle-antiparticle conjugation for relativistic field theories. Dan Mat Fys Medd 28(5):1-17

Lund MN, Silva Aguirre V, Davies GR, Chaplin WJ, Christensen-Dalsgaard J, Houdek G, White TR, Bedding TR, Ball WH, Huber D, Antia HM, Lebreton Y, Latham DW, Handberg R, Verma K, Basu S, Casagrande L, Justesen AB, Kjeldsen H, Mosumgaard JR (2017) Standing on the shoulders of dwarfs: the Kepler asteroseismic LEGACY Sample. I. Oscillation mode parameters. Astrophys J 835:172. https://doi.org/10.3847/1538-4357/835/2/172. arXiv:1612.00436

Maeda K, Shibahashi H (2014) Pulsations of pre-white dwarfs with hydrogen-dominated atmospheres. PASJ 66:76. https://doi.org/10.1093/pasj/psu051. arXiv:1405.4568

Malec B, Biesiada M (2013) White Dwarf Constraints on Exotic Physical Theories. In: 18th European White Dwarf Workshop, ASP Conference Series, vol 469. Astronomical Society of the Pacific, San Francisco, p 21

Maoz D, Mannucci F, Nelemans G (2014) Observational Clues to the Progenitors of Type Ia Supernovae. Annu Rev Astron Astrophys 52:107-170. https://doi.org/10.1146/annurev-astro-082812-141031. arXiv:1312.0628

Marino AF, Milone AP, Yong D, Da Costa G, Asplund M, Bedin LR, Jerjen H, Nardiello D, Piotto G, Renzini A, Shetrone M (2017) Spectroscopy and photometry of multiple populations along the ssymptotic giant branch of NGC 2808 and NGC 6121 (M4). Astrophys J 843:66. https://doi.org/10.3847/1538-4357/ aa7852

Maxted PFL, Anderson DR, Burleigh MR, Collier Cameron A, Heber U, Gänsicke BT, Geier S, Kupfer T, Marsh TR, Nelemans G, O’Toole SJ, Østensen RH, Smalley B, West RG (2011) Discovery of a stripped red giant core in a bright eclipsing binary system. Mon Not R Astron Soc 418:1156-1164. https://doi.org/10.1111/j.1365-2966.2011.19567.x. arXiv:1107.4986

Maxted PFL, Serenelli AM, Miglio A, Marsh TR, Heber U, Dhillon VS, Littlefair S, Copperwheat C, Smalley B, Breedt E, Schaffenroth V (2013) Multi-periodic pulsations of a stripped red-giant star in an eclipsing binary system. Nature 498:463-465. https://doi.org/10.1038/nature12192. arXiv:1307.1654

Maxted PFL, Bloemen S, Heber U, Geier S, Wheatley PJ, Marsh TR, Breedt E, Sebastian D et al (2014a) EL CVn-type binaries-discovery of 17 helium white dwarf precursors in bright eclipsing binary star systems. Mon Not R Astron Soc 437:1681-1697. https://doi.org/10.1093/mnras/stt2007. arXiv: 1310.4863

Maxted PFL, Serenelli AM, Marsh TR, Catalán S, Mahtani DP, Dhillon VS (2014b) WASP $1628+10$ - an EL CVn-type binary with a very low mass stripped red giant star and multiperiodic pulsations. Mon Not R Astron Soc 444:208-216. https://doi.org/10.1093/mnras/stu1465. arXiv:1407.5415 
McGraw JT, Starrfield SG, Liebert J, Green R (1979) PG1159-035: A new, hot, non-DA pulsating degenerate. In: van Horn HM, Weidemann V, Savedoff MP (eds) IAU Colloq. 53: White Dwarfs and Variable Degenerate Stars, University of Rochester, Rochester, NY, pp 377-381

Medin Z, Cumming A (2010) Crystallization of classical multicomponent plasmas. Phys Rev E 81(3):036107. https://doi.org/10.1103/PhysRevE.81.036107. arXiv:1002.3327

Mestel L (1952) On the theory of white dwarf stars. I. The energy sources of white dwarfs. Mon Not R Astron Soc 112:583. https://doi.org/10.1093/mnras/112.6.583

Metcalfe TS (2003) White dwarf asteroseismology and the ${ }^{12} \mathrm{C}(\alpha, \gamma){ }^{16} \mathrm{O}$ rate. Astrophys J Lett 587:L43L46. https://doi.org/10.1086/375044. arXiv:astro-ph/0303039

Metcalfe TS, Montgomery MH, Kanaan A (2004) Testing white dwarf crystallization theory with asteroseismology of the massive pulsating DA star BPM 37093. Astrophys J Lett 605:L133-L136. https:// doi.org/10.1086/420884. arXiv:astro-ph/0402046

Miller Bertolami MM, Althaus LG, Córsico AH (2017) On the Formation of DA White Dwarfs with low Hydrogen Contents: Preliminary Results. In: Tremblay PE, Gaensicke B, Marsh T (eds) 20th European White Dwarf Workshop, ASP Conference Series, vol 509. Astronomical Society of the Pacific, San Francisco, p 435. arXiv:1609.08683

Miller Bertolami MM (2014) Limits on the neutrino magnetic dipole moment from the luminosity function of hot white dwarfs. Astron Astrophys 562:A123. https://doi.org/10.1051/0004-6361/201322641. arXiv:1407.1404

Miller Bertolami MM (2016) New models for the evolution of post-asymptotic giant branch stars and central stars of planetary nebulae. Astron Astrophys 588:A25. https://doi.org/10.1051/0004-6361/ 201526577. arXiv:1512.04129

Miller Bertolami MM, Althaus LG (2006) Full evolutionary models for PG 1159 stars. Implications for the helium-rich $\mathrm{O}(\mathrm{He})$ stars. Astron Astrophys 454:845-854. https://doi.org/10.1051/0004-6361: 20054723. arXiv:astro-ph/0603846

Miller Bertolami MM, Althaus LG (2007) The born-again (very late thermal pulse) scenario revisited: the mass of the remnants and implications for V4334 Sgr. Mon Not R Astron Soc 380:763-770. https:// doi.org/10.1111/j.1365-2966.2007.12115.x. arXiv:0706.0714

Miller Bertolami MM, Althaus LG, Olano C, Jiménez N (2011) The diffusion-induced nova scenario: CK Vul and PB8 as possible observational counterparts. Mon Not R Astron Soc 415:1396-1408. https:// doi.org/10.1111/j.1365-2966.2011.18790.x. arXiv:1103.5455

Miller Bertolami MM, Althaus LG, García-Berro E (2013) Quiescent nuclear burning in lowmetallicity white dwarfs. Astrophys J 775(1):L22. https://doi.org/10.1088/2041-8205/775/1/L22. arXiv:1308.2062

Miller Bertolami MM, Melendez BE, Althaus LG, Isern J (2014) Revisiting the axion bounds from the Galactic white dwarf luminosity function. J Cosmol Astropart Phys 10:069. https://doi.org/10.1088/ 1475-7516/2014/10/069. arXiv:1406.7712

Montgomery MH, Winget DE (1999) The effect of crystallization on the pulsations of white dwarf stars. Astrophys J 526:976-990. https://doi.org/10.1086/308044. arXiv:astro-ph/9907040

Montgomery MH, Williams KA, Winget DE, Dufour P, DeGennaro S, Liebert J (2008) SDSS J142625.71+575218.3: a prototype for a new class of variable white dwarf. Astrophys J Lett 678:L51. https://doi.org/10.1086/588286. arXiv:0803.2646

Mosser B, Benomar O, Belkacem K, Goupil MJ, Lagarde N, Michel E, Lebreton Y, Stello D, Vrard M, Barban C, Bedding TR, Deheuvels S, Chaplin WJ, De Ridder J, Elsworth Y, Montalban J, Noels A, Ouazzani RM, Samadi R, White TR, Kjeldsen H (2014) Mixed modes in red giants: a window on stellar evolution. Astron Astrophys 572:L5. https://doi.org/10.1051/0004-6361/201425039. arXiv: 1411.1082

Mould J, Uddin SA (2014) Constraining a possible variation of G with type Ia Supernovae. PASA 31:e015. https://doi.org/10.1017/pasa.2014.9. arXiv:1402.1534

Mukadam AS, Mullally F, Nather RE, Winget DE, von Hippel T, Kleinman SJ, Nitta A, Krzesiński J, Kepler SO, Kanaan A, Koester D, Sullivan DJ, Homeier D, Thompson SE, Reaves D, Cotter C, Slaughter D, Brinkmann J (2004) Thirty-five new pulsating DA white dwarf stars. Astrophys J 607:982-998. https://doi.org/10.1086/383083

Mukadam AS, Bischoff-Kim A, Fraser O, Córsico AH, Montgomery MH, Kepler SO, Romero AD, Winget DE et al (2013) Measuring the evolutionary rate of cooling of ZZ Ceti. Astrophys J 771:17. https:// doi.org/10.1088/0004-637X/771/1/17 
Mullally F, Thompson SE, Castanheira BG, Winget DE, Kepler SO, Eisenstein DJ, Kleinman SJ, Nitta A (2005) Eleven new DA white dwarf variable stars from the Sloan Digital Sky Survey. Astrophys J 625:966-972. https://doi.org/10.1086/429885

Nandez JLA, Ivanova N (2016) Common envelope events with low-mass giants: understanding the energy budget. Mon Not R Astron Soc 460:3992-4002. https://doi.org/10.1093/mnras/stw1266. arXiv: 1606.04922

Nather RE, Winget DE, Clemens JC, Hansen CJ, Hine BP (1990) The Whole Earth Telescope: a new astronomical instrument. Astrophys J 361:309-317. https://doi.org/10.1086/169196

Nitta A, Kleinman SJ, Krzesinski J, Kepler SO, Metcalfe TS, Mukadam AS, Mullally F, Nather RE, Sullivan DJ, Thompson SE, Winget DE (2009) New pulsating DB white dwarf stars from the Sloan Digital Sky Survey. Astrophys J 690:560-565. https://doi.org/10.1088/0004-637X/690/1/560. arXiv:0809.0921

Nitta A, Kepler SO, Chené AN, Koester D, Provencal JL, Kleinmani SJ, Sullivan DJ, Chote P, Sefako R, Kanaan A, Romero A, Corti M, Kilic M, Montgomery MH, Winget DE (2016) Constraining the physics of carbon crystallization through pulsations of a massive DAV BPM37093. IAU Focus Meeting 29:493-496. https://doi.org/10.1017/S1743921316005962

Norris JE (2004) The helium abundances of $\omega$ Centauri. Astrophys J Lett 612:L25-L28. https://doi.org/10. $1086 / 423986$

O'Brien MS, Kawaler SD (2000) The predicted signature of neutrino emission in observations of pulsating pre-white dwarf stars. Astrophys J 539:372-378. https://doi.org/10.1086/309216. arXiv:astro-ph/0003261

Østensen RH, Silvotti R, Charpinet S, Oreiro R, Handler G, Green EM, Bloemen S, Heber U et al (2010) First Kepler results on compact pulsators-I. Survey target selection and the first pulsators. Mon Not R Astron Soc 409:1470-1486. https://doi.org/10.1111/j.1365-2966.2010.17366.x. arXiv:1007.3170

Østensen RH, Bloemen S, Vučković M, Aerts C, Oreiro R, Kinemuchi K, Still M, Koester D (2011a) At last - A V777 Her pulsator in the Kepler field. Astrophys J Lett 736:L39. https://doi.org/10.1088/20418205/736/2/L39

Østensen RH, Silvotti R, Charpinet S, Oreiro R, Bloemen S, Baran AS, Reed MD, Kawaler SD et al (2011) First Kepler results on compact pulsators - VI. Targets in the final half of the survey phase. Mon Not R Astron Soc 414:2860-2870. https://doi.org/10.1111/j.1365-2966.2011.18405.x. arXiv:1101.4150

Paczyński B (1971) Evolution of single stars. VI. Model nuclei of planetary nebulae. Acta Astron 21:417

Pauli W, Rosenfeld L, Weisskopf V (eds) (1955) Niels Bohr and the development of physics. Pergamon Press, New York

Paxton B, Bildsten L, Dotter A, Herwig F, Lesaffre P, Timmes F (2011) Modules for experiments in stellar astrophysics (MESA). Astrophys J Suppl 192:3. https://doi.org/10.1088/0067-0049/192/1/3. arXiv: 1009.1622

Paxton B, Cantiello M, Arras P, Bildsten L, Brown EF, Dotter A, Mankovich C, Montgomery MH, Stello D, Timmes FX, Townsend R (2013) Modules for experiments in stellar astrophysics (MESA): planets, oscillations, rotation, and massive stars. Astrophys J Suppl 208:4. https://doi.org/10.1088/0067-0049/ 208/1/4. arXiv:1301.0319

Paxton B, Marchant P, Schwab J, Bauer EB, Bildsten L, Cantiello M, Dessart L, Farmer R, Hu H, Langer N, Townsend RHD, Townsley DM, Timmes FX (2015) Modules for experiments in stellar astrophysics (MESA): binaries, pulsations, and explosions. Astrophys J Suppl 220:15. https://doi.org/10.1088/ 0067-0049/220/1/15. arXiv:1506.03146

Paxton B, Schwab J, Bauer EB, Bildsten L, Blinnikov S, Duffell P, Farmer R, Goldberg JA, Marchant P, Sorokina E, Thoul A, Townsend RHD, Timmes FX (2018) Modules for experiments in stellar astrophysics (MESA): convective boundaries, element diffusion, and massive star explosions. Astrophys J Suppl 234:34. https://doi.org/10.3847/1538-4365/aaa5a8. arXiv:1710.08424

Paxton B, Smolec R, Gautschy A, Bildsten L, Cantiello M, Dotter A, Farmer R, Goldberg JA, Jermyn AS, Kanbur SM, Marchant P, Schwab J, Thoul A, Townsend RHD, Wolf WM, Zhang M, Timmes FX (2019) Modules for Experiments in Stellar Astrophysics (MESA): Pulsating Variable Stars, Rotation, Convective Boundaries, and Energy Conservation. Astrophys J Suppl 243:10. https://doi.org/10.3847/ 1538-4365/ab2241. arXiv:1903.01426

Peccei RD, Quinn HR (1977) CP conservation in the presence of pseudoparticles. Phys Rev Lett 38:14401443. https://doi.org/10.1103/PhysRevLett.38.1440

Pelisoli I, Kepler SO, Koester D (2018a) The sdA problem-I. Physical properties. Mon Not R Astron Soc 475:2480-2495. https://doi.org/10.1093/mnras/sty011. arXiv:1801.00495 
Pelisoli I, Kepler SO, Koester D, Castanheira BG, Romero AD, Fraga L (2018b) The sdA problem-II. Photometric and spectroscopic follow-up. Mon Not R Astron Soc 478:867-884. https://doi.org/10. 1093/mnras/sty1101. arXiv:1804.09059

Pelisoli I, Bell KJ, Kepler SO, Koester D (2019) The sdA problem-III. New extremely low-mass white dwarfs and their precursors from Gaia astrometry. Mon Not R Astron Soc 482:3831-3842. https:// doi.org/10.1093/mnras/sty2979. arXiv:1805.04070

Pietrinferni A, Cassisi S, Salaris M, Castelli F (2004) A large stellar evolution database for population synthesis studies. I. Scaled solar models and isochrones. Astrophys J 612:168-190. https://doi.org/ 10.1086/422498. arXiv:astro-ph/0405193

Pietrukowicz P (2018) On the Properties of Blue Large-Amplitude Pulsators. No BLAPs in the Magellanic Clouds. In: Smolec R, Kinemuchi K, Anderson RI (eds) The RR Lyrae 2017 Conference. Revival of the Classical Pulsators: from Galactic Structure to Stellar Interior Diagnostics, vol 6, pp 258-262. arXiv: 1802.04405

Pietrukowicz P, Dziembowski WA, Latour M, Angeloni R, Poleski R, di Mille F, Soszyński I, Udalski A, Szymański MK, Wyrzykowski Ł, Kozłowski S, Skowron J, Skowron D, Mróz P, Pawlak M, Ulaczyk K (2017) Blue large-amplitude pulsators as a new class of variable stars. Nat Astron 1:0166. https:// doi.org/10.1038/s41550-017-0166. arXiv:1706.07802

Pringle JE (1975) Period changes in eruptive binaries. Mon Not R Astron Soc 170:633-642. https://doi. org/10.1093/mnras/170.3.633

Provencal JL, Montgomery MH, Kanaan A, Shipman HL, Childers D, Baran A, Kepler SO, Reed M et al (2009) 2006 Whole Earth Telescope observations of GD358: a new look at the prototype DBV. Astrophys J 693:564-585. https://doi.org/10.1088/0004-637X/693/1/564. arXiv:0811.0768

Pyrzas S, Gänsicke BT, Hermes JJ, Copperwheat CM, Rebassa-Mansergas A, Dhillon VS, Littlefair SP, Marsh TR, Parsons SG, Savoury CDJ, Schreiber MR, Barros SCC, Bento J, Breedt E, Kerry P (2015) Discovery of ZZ Cetis in detached white dwarf plus main-sequence binaries. Mon Not R Astron Soc 447:691-697. https://doi.org/10.1093/mnras/stu2412. arXiv:1411.5045

Quirion PO, Dupret MA, Fontaine G, Brassard P, Grigahcène A (2008) Hydrogen-Deficient Compact Pulsators: The GW Virginis Stars and the Variable DB White Dwarfs. In: Werner A, Rauch T (eds) Hydrogen-Deficient Stars, ASPConference Series, vol 391. Astronomical Society of the Pacific, San Francisco, p 183

Quirion PO, Fontaine G, Brassard P (2012) Wind competing against settling: a coherent model of the GW virginis instability domain. Astrophys J 755:128. https://doi.org/10.1088/0004-637X/755/2/128

Raffelt G (2012) Neutrinos and the stars. ArXiv e-prints arXiv:1201.1637

Raffelt GG (1986) Axion constraints from white dwarf cooling times. Phys Lett B 166:402-406. https:// doi.org/10.1016/0370-2693(86)91588-1

Raffelt GG (1990) Astrophysical methods to constrain axions and other novel particle phenomena. Phys Rep 198:1-113. https://doi.org/10.1016/0370-1573(90)90054-6

Raffelt GG (1996) Stars as laboratories for fundamental physics: the astrophysics of neutrinos, axions, and other weakly interacting particles. University of Chicago Press, Chicago

Raffelt GG (2007) Axions-motivation, limits and searches. J Phys A Math Gen 40:6607-6620. https://doi. org/10.1088/1751-8113/40/25/S05. arXiv:hep-ph/0611118

Ramsay G (2018) Identifying blue large-amplitude pulsators in the Galactic plane using Gaia DR2: a case study. Astron Astrophys 620:L9. https://doi.org/10.1051/0004-6361/201834604. arXiv:1811.09522

Redaelli M, Kepler SO, Costa JES, Winget DE, Handler G, Castanheira BG, Kanaan A, Fraga L et al (2011) The pulsations of PG 1351+489. Mon Not R Astron Soc 415:1220-1227. https://doi.org/10.1111/j. 1365-2966.2011.18743.x

Redondo J (2016) Axions at the international axion observatory. ArXiv e-prints arXiv:1601.00578

Ricker GR, Winn JN, Vanderspek R, Latham DW, Bakos GÁ, Bean JL, Berta-Thompson ZK, Brown TM et al (2015) Transiting exoplanet survey satellite (TESS). J Astron Telesc Instrum Syst 1(1):014003. https://doi.org/10.1117/1.JATIS.1.1.014003

Ritossa C, Garcia-Berro E, Iben I Jr (1996) On the evolution of stars that form electron-degenerate cores processed by carbon burning. II. Isotope abundances and thermal pulses in a $10 M_{\odot}$ model with an ONe core and applications to long-period variables, classical novae, and accretion-induced collapse. Astrophys J 460:489. https://doi.org/10.1086/176987

Romero AD, Córsico AH, Althaus LG, Kepler SO, Castanheira BG, Miller Bertolami MM (2012) Toward ensemble asteroseismology of ZZ Ceti stars with fully evolutionary models. Mon Not R Astron Soc 420:1462-1480. https://doi.org/10.1111/j.1365-2966.2011.20134.x 
Romero AD, Kepler SO, Córsico AH, Althaus LG, Fraga L (2013) Asteroseismological study of massive ZZ Ceti stars with fully evolutionary models. Astrophys J 779:58. https://doi.org/10.1088/0004-637X/ 779/1/58. arXiv: 1310.4137

Romero AD, Córsico AH, Castanheira BG, De Gerónimo FC, Kepler SO, Koester D, Kawka A, Althaus LG, Hermes JJ, Bonato C, Gianninas A (2017) Probing the structure of Kepler ZZ Ceti stars with full evolutionary models-based asteroseismology. Astrophys J 851:60. https://doi.org/10.3847/15384357/aa9899. arXiv:1711.01338

Romero AD, Córsico AH, Althaus LG, Pelisoli I, Kepler SO (2018) On the evolutionary status and pulsations of the recently discovered blue large-amplitude pulsators (BLAPs). Mon Not R Astron Soc 477:L30L34. https://doi.org/10.1093/mnrasl/sly051. arXiv:1803.09600

Rowan DM, Tucker MA, Shappee BJ, Hermes JJ (2019) Detections and constraints on white dwarf variability from time-series GALEX observations. Mon Not R Astron Soc 486:4574-4589. https://doi.org/10. 1093/mnras/stz1116. arXiv:1812.05614

Saio H (2019) r-mode oscillations in accreting white dwarfs in cataclysmic variables. Mon Not R Astron Soc. https://doi.org/10.1093/mnras/stz1407. arXiv:1905.08390

Salaris M, Cassisi S (2018) White dwarf stars: cosmic chronometers and dark matter probes. Physica Scr 93(4):044002. https://doi.org/10.1088/1402-4896/aaaef4

Salaris M, Serenelli A, Weiss A, Miller Bertolami M (2009) Semi-empirical white dwarf initial-final mass relationships: a thorough analysis of systematic uncertainties due to stellar evolution models. Astrophys J 692:1013-1032. https://doi.org/10.1088/0004-637X/692/2/1013. arXiv:0807.3567

Schoenberner D (1987) Mass loss and the transformation of AGB stars into central stars of planetary nebulae. In: Kwok S, Pottasch SR (eds) Late Stages of Stellar Evolution, Astrophysics and Space Science Library, vol 132. D. Reidel, Dordrecht

Schwarzschild M (1958) Structure and evolution of the stars. Princeton University Press, Princeton

Scuflaire R (1974) The non radial oscillations of condensed polytropes. Astron Astrophys 36:107

Segretain L, Chabrier G (1993) Crystallization of binary ionic mixtures in dense stellar plasmas. Astron Astrophys 271:L13

Serenelli AM, Althaus LG, Rohrmann RD, Benvenuto OG (2002) Evolution and colours of helium-core white dwarf stars: the case of low-metallicity progenitors. Mon Not R Astron Soc 337:1091-1104. https://doi.org/10.1046/j.1365-8711.2002.05994.x. arXiv:astro-ph/0208408

Shibahashi H (2005) The DB gap and pulsations of white dwarfs. In: Alecian G, Richard O, Vauclair S (eds) Element stratification in stars: 40 years of atomic diffusion, EAS Publications Series, vol 17. EDP sciences, pp 143-148. https://doi.org/10.1051/eas:2005108

Shibahashi H (2007) The DB Gap and Pulsations of White Dwarfs. In: Stancliffe RJ, Houdek G, Martin RG, Tout CA (eds) Unsolved problems in stellar physics: a conference in honor of Douglas Gough, ASP Conference Series, vol 948. American Institute of Physics, pp 35-42. https://doi.org/10.1063/1. 2818994

Shibahashi H (2013) A new kind of pulsator in the DB valley of white dwarf stars. In: Alecian G, Lebreton Y, Richard O, Vauclair G (eds) EAS Publications Series, EAS Publications Series, vol 63, pp 185-190. https://doi.org/10.1051/eas/1363021

Siess L (2010) Evolution of massive AGB stars. III. The thermally pulsing super-AGB phase. Astron Astrophys 512:A10. https://doi.org/10.1051/0004-6361/200913556

Silvotti R, Østensen RH, Bloemen S, Telting JH, Heber U, Oreiro R, Reed MD, Farris LE, O'Toole SJ, Lanteri L, Degroote P, Hu H, Baran AS, Hermes JJ, Althaus LG, Marsh TR, Charpinet S, Li J, Morris RL, Sanderfer DT (2012) Orbital properties of an unusually low-mass sdB star in a close binary system with a white dwarf. Mon Not R Astron Soc 424:1752-1761. https://doi.org/10.1111/j.13652966.2012.21232.x. arXiv:1205.2457

Sion EM (2011) Hot white dwarfs. In: Hoard DW (ed) White dwarf atmospheres and circumstellar environments. Wiley-VCH, Weinheim, pp 1-24

Smolec R, Pietrzyński G, Graczyk D, Pilecki B, Gieren W, Thompson I, Stępień K, Karczmarek P, Konorski P, Górski M, Suchomska K, Bono G, Prada PGM, Nardetto N (2013) Pulsation models for the $0.26 M_{\odot}$ star mimicking RR Lyrae pulsator. Model survey for the new class of variable stars. Mon Not R Astron Soc 428:3034-3047. https://doi.org/10.1093/mnras/sts258. arXiv:1210.6030

Sowicka P, Handler G, Jones D (2018) On $\epsilon$-mechanism-driven pulsations in VV 47. Mon Not R Astron Soc 479:2476-2480. https://doi.org/10.1093/mnras/sty1660. arXiv:1806.07935

Starrfield SG, Cox AN, Hodson SW, Pesnell WD (1983) The discovery of nonradial instability strips for hot, evolved stars. Astrophys J Lett 268:L27-L32. https://doi.org/10.1086/184023 
Starrfield S, Cox AN, Kidman RB, Pesnell WD (1984) Nonradial instability strips based on carbon and oxygen partial ionization in hot, evolved stars. Astrophys J 281:800-810. https://doi.org/10.1086/ 162158

Steinfadt JDR, Bildsten L, Arras P (2010) Pulsations in hydrogen burning low-mass helium white dwarfs. Astrophys J 718:441-445. https://doi.org/10.1088/0004-637X/718/1/441. arXiv:1005.5423

Steinfadt JDR, Bildsten L, Kaplan DL, Fulton BJ, Howell SB, Marsh TR, Ofek EO, Shporer A (2012) A search for pulsations in helium white dwarfs. PASP 124:1-13. https://doi.org/10.1086/663865. arXiv: 1105.0472

Strickler RR, Cool AM, Anderson J, Cohn HN, Lugger PM, Serenelli AM (2009) Helium-core white dwarfs in the globular cluster NGC 6397. Astrophys J 699:40-55. https://doi.org/10.1088/0004-637X/699/ 1/40. arXiv:0904.3496

Sullivan DJ, Chote P (2015) The frequency stability of the pulsating white dwarf L19-2. In: Dufour P, Bergeron P, Fontaine G (eds) 19th European workshop on white dwarfs, Astronomical Society of the Pacific, San Francisco, ASP Conference Series, vol 493, p 199

Sullivan DJ (2017) Time-series spectroscopy and photometry of the helium atmosphere pulsating white dwarf EC 20058-5234. In: Tremblay PE, Gaensicke B, Marsh T (eds) 20th European white dwarf workshop, Astronomical Society of the Pacific, San Francisco, ASP Conference Series, vol 509, p 315

Szkody P, Mukadam A, Gänsicke BT, Henden A, Templeton M, Holtzman J, Montgomery MH, Howell SB, Nitta A, Sion EM, Schwartz RD, Dillon W (2010) Finding the instability strip for accreting pulsating white dwarfs from hubble space telescope and optical observations. Astrophys J 710:64-77. https:// doi.org/10.1088/0004-637X/710/1/64. arXiv:1001.0192

Szkody P, Mukadam AS, Gänsicke BT, Hermes JJ, Toloza O (2015) An update on the quirks of pulsating, accreting white dwarfs. In: Dufour P, Bergeron P, Fontaine G (eds) 19th European workshop on white dwarfs, Astronomical Society of the Pacific, San Francisco, ASP Conference Series, vol 493, p 205

Szkody P, Mukadam AS, Gänsicke BT, Sion EM, Townsley D, Henden A (2013) Enigmas of accreting pulsating white dwarfs. In: 18th European white dwarf workshop, Astronomical Society of the Pacific, San Francisco, ASP Conference Series, vol 469, p 31

Tassoul M, Fontaine G, Winget DE (1990) Evolutionary models for pulsation studies of white dwarfs. Astrophys J Suppl Ser 72:335-386. https://doi.org/10.1086/191420

Tayar J, Pinsonneault MH (2013) Implications of rapid core rotation in red giants for internal angular momentum transport in stars. Astrophys J Lett 775:L1. https://doi.org/10.1088/2041-8205/775/1/L1. arXiv:1306.3986

Thompson SE, Provençal JL, Kanaan A, Montgomery MH, Bishoff-Kim A, Shipman H, Team Wet (2009) Whole Earth Telescope observations of the DAVs R808 and G38-29. J Phys Conf Ser 172:012067. https://doi.org/10.1088/1742-6596/172/1/012067

Toloza O, Gänsicke BT, Hermes JJ, Townsley DM, Schreiber MR, Szkody P, Pala A, Beuermann K, Bildsten L, Breedt E, Cook M, Godon P, Henden AA, Hubeny I, Knigge C, Long KS, Marsh TR, de Martino D, Mukadam AS, Myers G, Nelson P, Oksanen A, Patterson J, Sion EM, Zorotovic M (2016) GW Librae: a unique laboratory for pulsations in an accreting white dwarf. Mon Not R Astron Soc 459:3929-3938. https://doi.org/10.1093/mnras/stw838. arXiv:1604.02162

Tremblay PE, Bergeron P (2008) The ratio of helium- to hydrogen-atmosphere white dwarfs: direct evidence for convective mixing. Astrophys J 672:1144-1152. https://doi.org/10.1086/524134. arXiv:0710.1073

Tremblay PE, Ludwig HG, Steffen M, Freytag B (2013) Pure-hydrogen 3D model atmospheres of cool white dwarfs. Astron Astrophys 552:A13. https://doi.org/10.1051/0004-6361/201220813. arXiv:1302.2013

Tremblay PE, Ludwig HG, Steffen M, Freytag B (2013b) Spectroscopic analysis of DA white dwarfs with 3D model atmospheres. Astron Astrophys 559:A104. https://doi.org/10.1051/0004-6361/201322318. arXiv: 1309.0886

Tremblay PE, Gianninas A, Kilic M, Ludwig HG, Steffen M, Freytag B, Hermes JJ (2015) 3D model atmospheres for extremely low-mass white dwarfs. Astrophys J 809:148. https://doi.org/10.1088/ 0004-637X/809/2/148. arXiv:1507.01927

Tremblay PE, Fontaine G, Fusillo NPG, Dunlap BH, Gänsicke BT, Hollands MA, Hermes JJ, Marsh TR, Cukanovaite E, Cunningham T (2019) Core crystallization and pile-up in the cooling sequence of evolving white dwarfs. Nature 565:202-205. https://doi.org/10.1038/s41586-018-0791-X

Tucker MA, Fleming SW, Pelisoli I, Romero A, Bell KJ, Kepler SO, Caton DB, Debes J, Montgomery MH, Thompson SE, Koester D, Million C, Shiao B (2018) White dwarf variability with gPhoton: pulsators. Mon Not R Astron Soc 475(4):4768-4780. https://doi.org/10.1093/mnras/stx3297 
Udalski A, Szymański MK, Szymański G (2015) OGLE-IV: fourth phase of the optical gravitational lensing experiment. Acta Astron 65:1-38 arXiv:1504.05966

Unno W, Osaki Y, Ando H, Saio H, Shibahashi H (1989) Nonradial oscillations of stars, 2nd edn. University of Tokyo Press, Tokyo

Uzan JP (2003) The fundamental constants and their variation: observational and theoretical status. Rev Mod Phys 75:403-455. https://doi.org/10.1103/RevModPhys.75.403. arXiv:hep-ph/0205340

Van Grootel V, Dupret MA, Fontaine G, Brassard P, Grigahcène A, Quirion PO (2012) The instability strip of ZZ Ceti white dwarfs. I. Introduction of time-dependent convection. Astron Astrophys 539:A87. https://doi.org/10.1051/0004-6361/201118371

Van Grootel V, Fontaine G, Brassard P, Dupret MA (2013) The newly discovered pulsating low-mass white dwarfs: an extension of the ZZ Ceti instability strip. Astrophys J 762:57. https://doi.org/10.1088/0004$637 \mathrm{X} / 762 / 1 / 57$

Van Grootel V, Fontaine G, Brassard P, Dupret MA (2015) A connection between the instability strips of ZZ Ceti and V777 Herculis white dwarfs. Pulsating accreting GW Lib white dwarfs. Astron Astrophys 575:A125. https://doi.org/10.1051/0004-6361/201425386

Van Grootel V, Fontaine G, Brassard P, Dupret MA (2017) The theoretical instability strip of V777 Her white dwarfs. In: Tremblay PE, Gaensicke B, Marsh T (eds) 20th European white dwarf workshop, ASP Conference Series, vol 509. Astronomical Society of the Pacific, San Francisco, p 321

van Horn HM (1968) Crystallization of white dwarfs. Astrophys J 151:227. https://doi.org/10.1086/149432

Vanderbosch ZP, Winget KI, Winget DE (2018) New DBVs. In: 21th European White dwarf workshop (in preparation)

Vauclair G (2013) Constraints on white dwarfs structure and evolution from asteroseismology. In: Alecian G, Lebreton Y, Richard O, Vauclair G (eds) New advances in stellar physics: from microscopic to macroscopic processes, EAS Publications Series, vol 63. EDP Sciences, pp 175-183. https://doi.org/ $10.1051 /$ eas/1363020

Vauclair G, Fu JN, Solheim JE, Kim SL, Dolez N, Chevreton M, Chen L, Wood MA, Silver IM, Bognár Z, Paparó M, Córsico AH (2011) The period and amplitude changes in the coolest GW Virginis variable star (PG 1159-type) PG 0122+200. Astron Astrophys 528:A5. https://doi.org/10.1051/0004-6361/ 201014457

Vennes S, Nemeth P, Kawka A, Thorstensen JR, Khalack V, Ferrario L, Alper EH (2017) An unusual white dwarf star may be a surviving remnant of a subluminous Type Ia supernova. Science 357:680-683. https://doi.org/10.1126/science.aam8378. arXiv:1708.05568

Ventura P, D’Antona F, Mazzitelli I, Gratton R (2001) Predictions for self-pollution in globular cluster stars. Astrophys J Lett 550:L65-L69. https://doi.org/10.1086/319496. arXiv:astro-ph/0103337

Viaux N, Catelan M, Stetson PB, Raffelt GG, Redondo J, Valcarce AAR, Weiss A (2013a) Neutrino and axion bounds from the globular cluster M5 (NGC 5904). Phys Rev Lett 111(23):231301. https://doi. org/10.1103/PhysRevLett.111.231301. arXiv:1311.1669

Viaux N, Catelan M, Stetson PB, Raffelt GG, Redondo J, Valcarce AAR, Weiss A (2013b) Particle-physics constraints from the globular cluster M5: neutrino dipole moments. Astron Astrophys 558:A12. https:// doi.org/10.1051/0004-6361/201322004. arXiv:1308.4627

Voss B, Koester D, Østensen R, Napiwotzki R, Homeier D, Reimers D (2007) Six new ZZ Ceti stars from the SPY and the HQS surveys. In: Napiwotzki R, Burleigh MR (eds) 15th European workshop on white dwarfs, ASP Conference Series, vol 372. Astronomical Society of the Pacific, San Francisco, p 583, arXiv:0704.2710

Vysotsky MI, YaB Zeldovich, Khlopov MYu, Chechetkin VM (1978) Some astrophysical limitations on axion mass. Pisma Zh Eksp Teor Fiz 27:533-536 [JETP Lett.27,502(1978)]

Wachlin FC, Vauclair G, Vauclair S, Althaus LG (2017) Importance of fingering convection for accreting white dwarfs in the framework of full evolutionary calculations: the case of the hydrogen-rich white dwarfs GD 133 and G 29-38. Astron Astrophys 601:A13. https://doi.org/10.1051/0004-6361/ 201630094. arXiv:1612.09320

Warner B, van Zyl L (1998) Discovery of non-radial pulsations in the white dwarf primary of a cataclysmic variable star. In: Deubner FL, Christensen-Dalsgaard J, Kurtz D (eds) New eyes to see inside the sun and stars, IAU symposium, vol 185. Kluwer, Dordrecht, p 321, arXiv:cond-mat/9701105

Weinberg S (1978) A new light boson? Phys Rev Lett 40:223-226. https://doi.org/10.1103/PhysRevLett. 40.223

Werner K, Herwig F (2006) The elemental abundances in bare planetary nebula central stars and the shell burning in AGB stars. PASP 118:183-204. https://doi.org/10.1086/500443. arXiv:astro-ph/0512320 
Wilczek F (1978) Problem of strong P and T invariance in the presence of instantons. Phys Rev Lett 40:279-282. https://doi.org/10.1103/PhysRevLett.40.279

Williams KA, Winget DE, Montgomery MH, Dufour P, Kepler SO, Hermes JJ, Falcon RE, Winget KI, Bolte M, Rubin KHR, Liebert J (2013) Photometric variability in a warm, strongly magnetic DQ white dwarf, SDSS J103655.39+652252.2. Astrophys J 769:123. https://doi.org/10.1088/0004-637X/769/ 2/123. arXiv: 1304.3165

Williams KA, Montgomery MH, Winget DE, Falcon RE, Bierwagen M (2016) Variability in hot carbondominated atmosphere (Hot DQ) white dwarfs: rapid rotation? Astrophys J 817:27. https://doi.org/ 10.3847/0004-637X/817/1/27. arXiv:1511.08834

Winget DE (1982) Gravity mode instabilities in DA white dwarfs. PhD thesis, The University of Rochester Winget DE (1988) Seismological investigations of compact stars. In: Christensen-Dalsgaard J, Frandsen $\mathrm{S}$ (eds) Advances in helio- and asteroseismology, IAU symposium, vol 123. D. Reidel, Dordrecht, p 305. https://doi.org/10.1007/978-94-009-4009-3_67

Winget DE, Kepler SO (2008) Pulsating white dwarf stars and precision asteroseismology. Annu Rev Astron Astrophys 46:157-199. https://doi.org/10.1146/annurev.astro.46.060407.145250. arXiv:0806.2573

Winget DE, Robinson EL, Nather RD, Fontaine G (1982a) Photometric observations of GD 358: DB white dwarfs do pulsate. Astrophys J Lett 262:L11-L15. https://doi.org/10.1086/183902

Winget DE, van Horn HM, Tassoul M, Fontaine G, Hansen CJ, Carroll BW (1982b) Hydrogen-driving and the blue edge of compositionally stratified ZZ Ceti star models. Astrophys J Lett 252:L65-L68. https://doi.org/10.1086/183721

Winget DE, Hansen CJ, van Horn HM (1983) Do pulsating PG1159-035 stars put constraints on stellar evolution? Nature 303:781-782. https://doi.org/10.1038/303781a0

Winget DE, Nather RE, Clemens JC, Provencal J, Kleinman SJ, Bradley PA, Wood MA, Claver CF et al (1991) Asteroseismology of the DOV star PG 1159-035 with the Whole Earth Telescope. Astrophys J 378:326-346. https://doi.org/10.1086/170434

Winget DE, Nather RE, Clemens JC, Provencal JL et al (1994) Whole Earth Telescope observations of the DBV white dwarf GD 358. Astrophys J 430:839-849. https://doi.org/10.1086/174455

Winget DE, Sullivan DJ, Metcalfe TS, Kawaler SD, Montgomery MH (2004) A strong test of electroweak theory using pulsating DB white dwarf stars as plasmon neutrino detectors. Astrophys J Lett 602:L109_ L112. https://doi.org/10.1086/382591. arXiv:astro-ph/0312303

Winget DE, Kepler SO, Campos F, Montgomery MH, Girardi L, Bergeron P, Williams K (2009) The physics of crystallization from globular cluster white dwarf stars in NGC 6397. Astrophys J Lett 693:L6-L10. https://doi.org/10.1088/0004-637X/693/1/L6. arXiv:0901.2950

Woosley SE, Heger A (2015) The remarkable deaths of 9-11 solar mass stars. Astrophys J 810:34. https:// doi.org/10.1088/0004-637X/810/1/34. arXiv:1505.06712

Woudt PA, Warner B, Zietsman E (2012) SDSS J0349-0059 is a GW Virginis star. Mon Not R Astron Soc 426:2137-2141. https://doi.org/10.1111/j.1365-2966.2012.21899.x. arXiv:1208.1844

Wu Y, Goldreich P (2001) Gravity modes in ZZ Ceti stars. IV. Amplitude saturation by parametric instability. Astrophys J 546:469-483. https://doi.org/10.1086/318234. arXiv:astro-ph/0003163

York DG, Adelman J, Anderson JE Jr, Anderson SF, Annis J, Bahcall NA, Bakken JA, Barkhouser R, others (SDSS Collaboration) (2000) The Sloan Digital Sky Survey: technical summary. Astron J 120:1579-1587. https://doi.org/10.1086/301513. arXiv:astro-ph/0006396

Zhang XB, Fu JN, Li Y, Ren AB, Luo CQ (2016) Multi-period g-mode pulsations of a pre-He-WD Star in the eclipsing binary KIC 9164561. Astrophys J Lett 821:L32. https://doi.org/10.3847/2041-8205/ 821/2/L32

Zhitnitsky AR (1980) On possible duppression of the axion hadron interactions (In Russian). Sov J Nucl Phys 31:260 [Yad. Fiz. 31:497 (1980)]

Zong W, Charpinet S, Vauclair G (2016a) Signatures of nonlinear mode interactions in the pulsating hot B subdwarf star KIC 10139564. Astron Astrophys 594:A46. https://doi.org/10.1051/0004-6361/ 201629132. arXiv:1607.06621

Zong W, Charpinet S, Vauclair G, Giammichele N, Van Grootel V (2016b) Amplitude and frequency variations of oscillation modes in the pulsating DB white dwarf star KIC 08626021. The likely signature of nonlinear resonant mode coupling. Astron Astrophys 585:A22. https://doi.org/10.1051/0004-6361/ 201526300. arXiv: 1510.06884

Publisher's Note Springer Nature remains neutral with regard to jurisdictional claims in published maps and institutional affiliations. 